Supporting Information for

\title{
Generation of an Elusive Permanent Merocyanine via a Unique Mechanochemical Reaction Pathway
}

\author{
Molly E. McFadden and Maxwell J. Robb* \\ Division of Chemistry and Chemical Engineering, California Institute of Technology, Pasadena, \\ California 91125, United States \\ *E-mail: mrobb@caltech.edu
}

\section{Table of Contents}

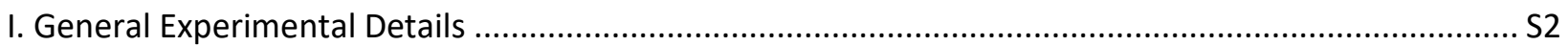

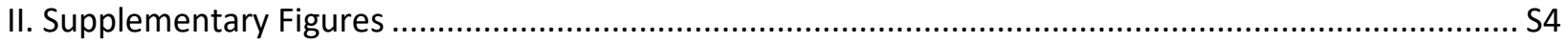

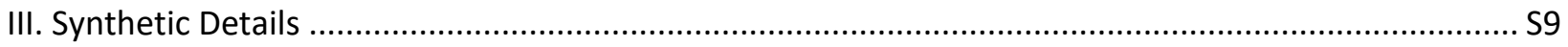

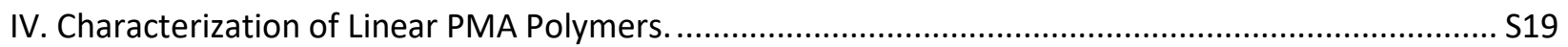

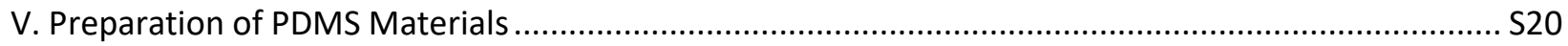

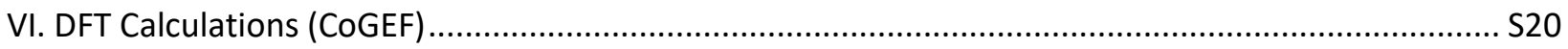

VII. Details for Photoirradiation and Sonication Experiments .......................................................... S21

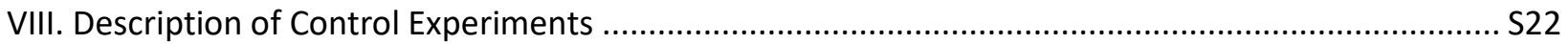

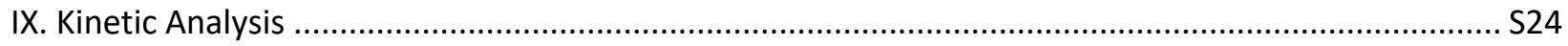

X. Characterization of Activation and Fading in PDMS Materials ......................................................... S25

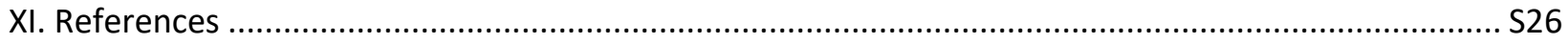

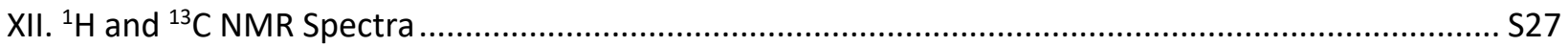




\section{General Experimental Details}

Reagents from commercial sources were used without further purification unless otherwise stated. Methyl acrylate was passed through a short plug of basic alumina to remove inhibitor immediately prior to use. Dry THF was obtained from a Pure Process Technology solvent purification system. All reactions were performed under a $\mathrm{N}_{2}$ or argon atmosphere unless specified otherwise. Column chromatography was performed on a Biotage Isolera system using SiliCycle SiliaSep HP flash cartridges.

NMR spectra were recorded using a $400 \mathrm{MHz}$ Bruker Avance III HD with Prodigy Cryoprobe. All ${ }^{1} \mathrm{H}$ NMR spectra are reported in $\delta$ units, parts per million ( $\mathrm{ppm})$, and were measured relative to the signals for residual $\mathrm{CHCl}_{3}$ (7.26 ppm), acetone (2.05 ppm), or acetonitrile (1.94 ppm) in deuterated solvent. All ${ }^{13} \mathrm{C}$ NMR spectra were measured in deuterated solvents and are reported in ppm relative to the signals for ${ }^{13} \mathrm{CDCl}_{3}$ (77.16 ppm) or acetone- $d_{6}(206.26 \mathrm{ppm})$. Multiplicity and qualifier abbreviations are as follows: $\mathrm{s}$ $=$ singlet, $\mathrm{d}=$ doublet, $\mathrm{m}=$ multiplet, $\mathrm{br}=$ broad, $\mathrm{app}=$ apparent.

High resolution mass spectra (HRMS) were obtained from a Waters Corp. LCT Premier XE time-of-flight mass spectrometer equipped with an electrospray ionization (ESI) probe, or a JEOL JMS-600H magnetic sector mass spectrometer equipped with a $F A B+$ probe.

Analytical gel permeation chromatography (GPC) was performed using an Agilent 1260 series pump equipped with two Agilent PLgel MIXED-B columns $(7.5 \times 300 \mathrm{~mm})$, an Agilent 1200 series diode array detector, a Wyatt 18-angle DAWN HELEOS light scattering detector, and a Optilab rEX differential refractive index detector. The mobile phase was THF at a flow rate of $1 \mathrm{~mL} / \mathrm{min}$. Molecular weights and molecular weight distributions were calculated by light scattering using a dn/dc value of $0.062 \mathrm{~mL} / \mathrm{g}(25$ $\left.{ }^{\circ} \mathrm{C}\right)$ for poly(methyl acrylate).

UV-Vis absorption spectra were recorded on a Thermo Scientific Evolution 220 spectrometer. Reflection measurements were performed on a Cary 5000 UV/Vis/NIR spectrophotometer equipped with an integrating sphere diffuse reflectance accessory (Internal DRA 1800). All reflection measurements were referenced to a LabSphere Spectralon 99\% certified reflectance standard. The samples were illuminated through a Spectralon-coated aperture with a diameter of $1 \mathrm{~cm}$, with a beam area of approximately 0.5 $\mathrm{cm}^{2}$.

Ultrasound experiments were performed using a Vibra Cell 505 liquid processor equipped with a 0.5-inch diameter solid probe (part \#630-0217), sonochemical adapter (part \#830-00014), and a Suslick reaction vessel made by the Caltech glass shop (analogous to vessel \#830-00014 from Sonics and Materials). Polymer solutions were continuously sampled for UV-vis analysis using a Cole Parmer Masterflex L/S pump system (item \#EW-77912-10) composed of an L/S pump head (part \#77390-00) and L/S precision variable speed drive (part \#07528-20) using 4x6 mm PTFE tubing (part \#77390-60) and a quartz flow-through cell (Starna, part \#583.4-Q-10/Z8.5), which was connected using M6-threaded PTFE tubing (Starna, part \#M6SET). 
Photoirradiation with UV light was performed using either a Philips PL-S 9W/01/2P UVB bulb with a narrow emission of 305-315 nm and a peak at $311 \mathrm{~nm}$, or a DR/9W-UVA bulb with peak at $365 \mathrm{~nm}$ under ambient conditions unless indicated otherwise. Irradiation with white light was carried out using a $13 \mathrm{~W}$ broadband fluorescent lamp (Bayco Model BA-506) filtered through a $425 \mathrm{~nm}$ bandpass filter. Irradiation with blue light was applied to PDMS films using a $470 \mathrm{~nm}$ LED (ThorLabs M470L3), driver (ledd1B), and collimator (SM1U25-A). 


\section{Supplementary Figures}

(a) Spectral evolution during sonication of $\mathbf{P 1}$

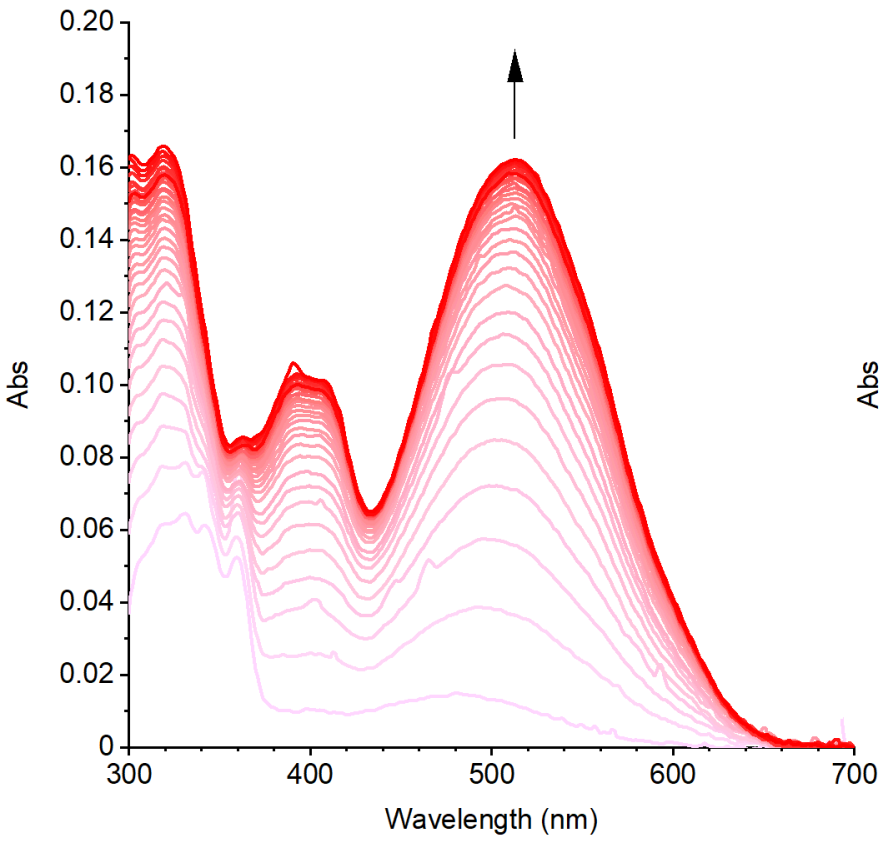

(b) Time-dependent absorbance at $510 \mathrm{~nm}$ during sonication of P1

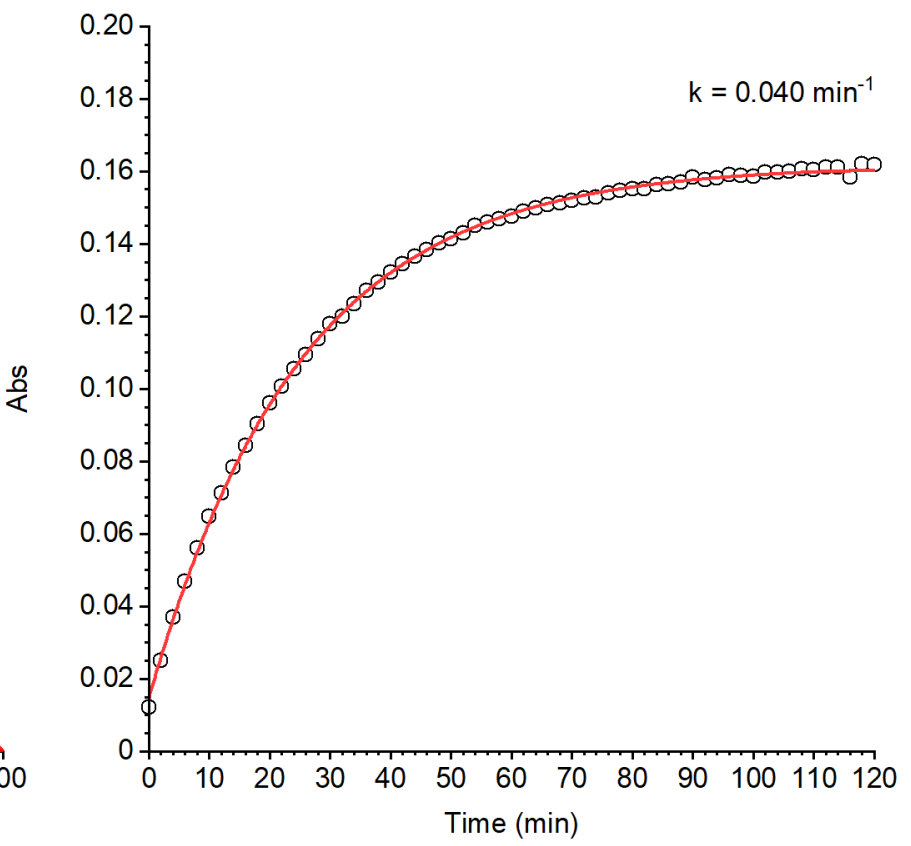

(c) Spectral evolution during sonication of P3

(d) Time-dependent absorbance at $470 \mathrm{~nm}$ during sonication of P3
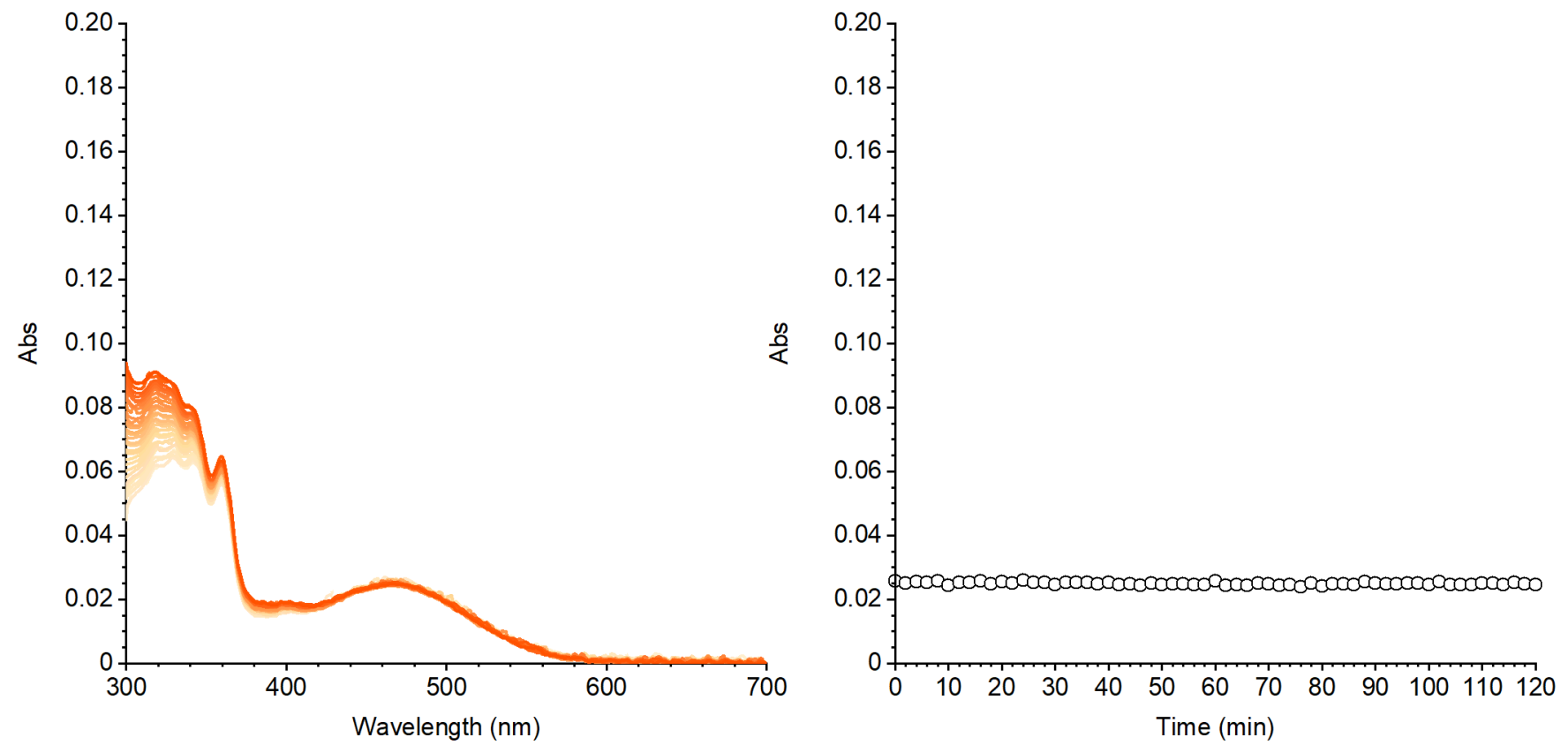

Figure S1. Comparison of sonication experiments performed on solutions ( $2 \mathrm{mg} / \mathrm{mL}$ in THF with $30 \mathrm{mM}$ BHT) of polymer P1 containing a chain-centered mechanophore and chain-end functional control polymer P3. (a) UV-vis spectra acquired at regular intervals during ultrasound-induced mechanical activation of $\mathbf{P} 1$ exhibit an increase in visible absorption with $\lambda_{\text {max }}$ of $510 \mathrm{~nm}$. (b) Fitting time-dependent absorbance at $510 \mathrm{~nm}$ to eq S1 yields a rate constant for merocyanine formation of 0.040 $\mathrm{min}^{-1}$. (c,d) No change in visible absorption is observed upon sonication of a solution of chain-end functional control polymer P3. 
(a) On-Off Cycles

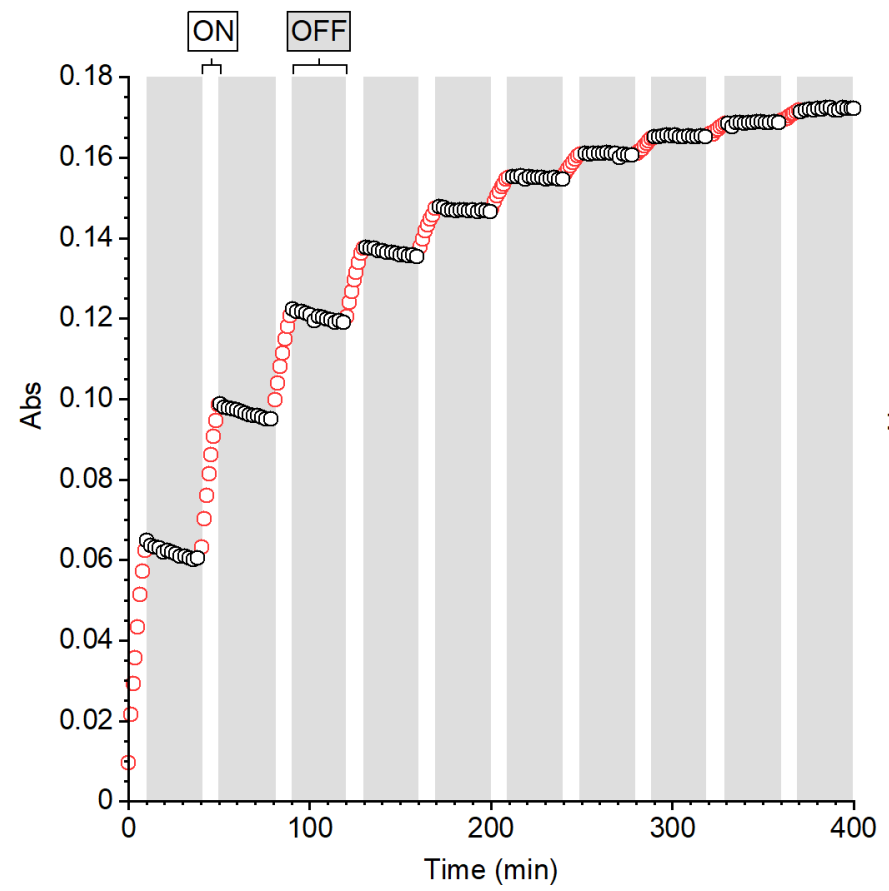

(b) Comparison of "on" cycles to continuous sonication

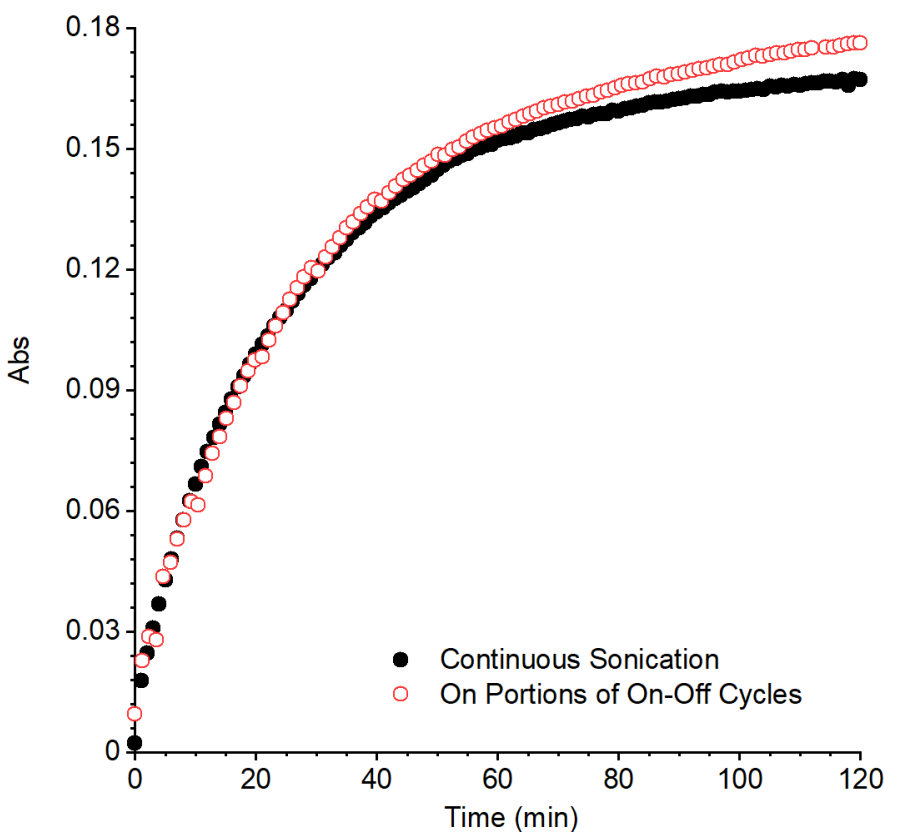

Figure S2. Investigation of the temporal dependence of permanent merocyanine formation. (a) A solution of P1 (2 mg/mL with $30 \mathrm{mM} \mathrm{BHT)}$ was exposed to "on-off" cycles of ultrasonication, alternating between 10 min "on" and 30 min "off". (b) Minimal loss of color is observed during the "off" periods, as demonstrated by comparing only the "on" time from panel a to the mechanochemical activation of P1 under continuous ultrasonication. Absorbance measured at $510 \mathrm{~nm}$.

(a) 10 s UV Irradiation: Spectral evolution

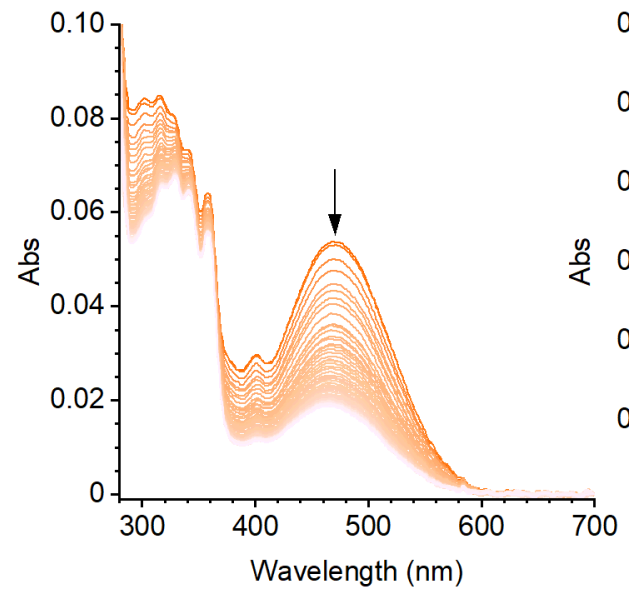

(b) $10 \mathrm{~s}$ UV Irradiation: $\mathrm{Abs}_{470 \mathrm{~nm}}$ vs Time

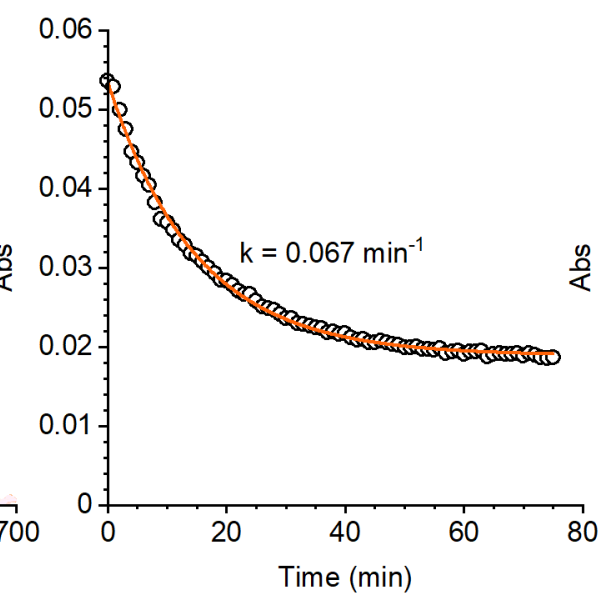

(c) 10 min UV Irradiation: Abs $_{470 \mathrm{~nm}}$ vs Time

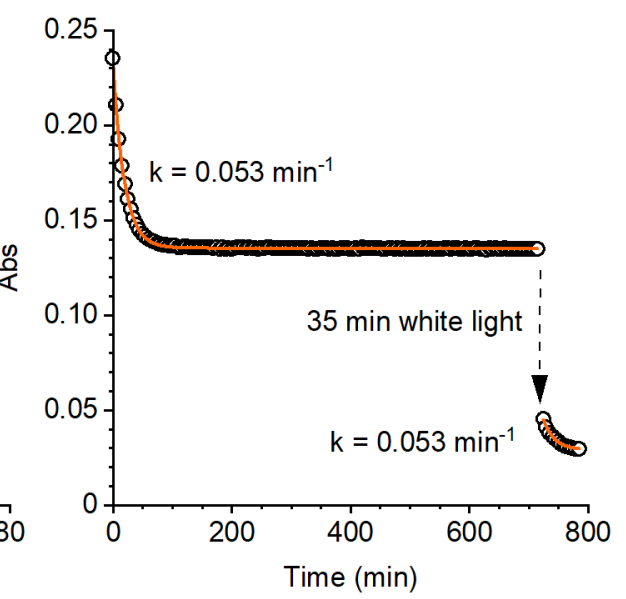

Figure S3. Demonstration of reversion of the photochemically generated merocyanine under ambient conditions or upon irradiation with white light. $(a, b)$ Brief photoactivation of a solution of P1 with UV light (10 s) produces a merocyanine with $\lambda_{\max }$ of $470 \mathrm{~nm}$ that fully reverts after approximately $1 \mathrm{~h}$ at room temperature. Fitting the time dependent absorbance at 470 $\mathrm{nm}$ to eq S2 yields a reversion rate of $0.067 \mathrm{~min}^{-1}$. (c) Extended irradiation of a solution of P1 with UV light (10 min) generates a merocyanine with the same $\lambda_{\max }$ of $470 \mathrm{~nm}$, which fades to half the initial absorbance in approximately $1 \mathrm{~h}$, leaving a persistent merocyanine attributed to the exocyclic trans isomer. Subsequent irradiation with white light for 35 min leads to additional reversion. Further thermal reversion occurs with the same rate constant observed initially. 
(a) Sonicated P1 + White Light

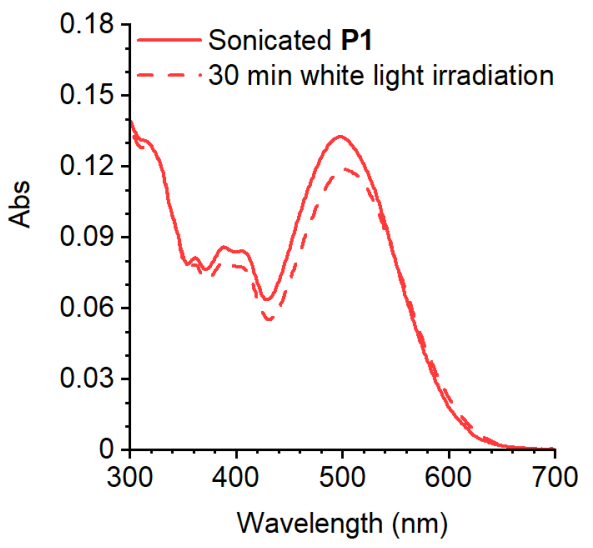

(b) M1 + White Light

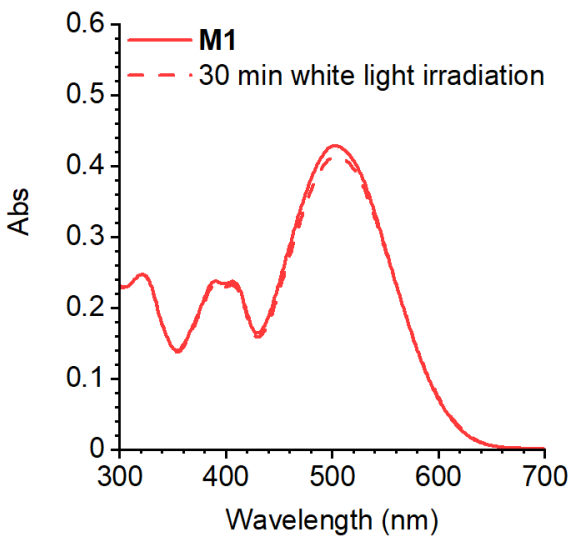

(c) UV Photoirradiated P1 + White Light

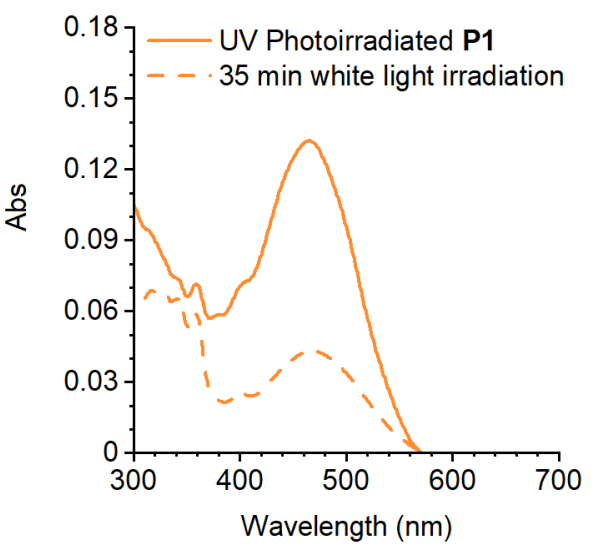

Figure S4. Irradiation of (a) the merocyanine derived from ultrasound-induced mechanochemical activation of P1 and (b) M1 with white light for 30 min results in negligible (<10\%) change in visible absorption. (c) Irradiation of the thermally persistent merocyanine generated from photochemical activation of P1 with white light for 35 min under the same conditions results in $67 \%$ attenuation of the visible absorbance at $470 \mathrm{~nm}$ (see Figure S3c above).

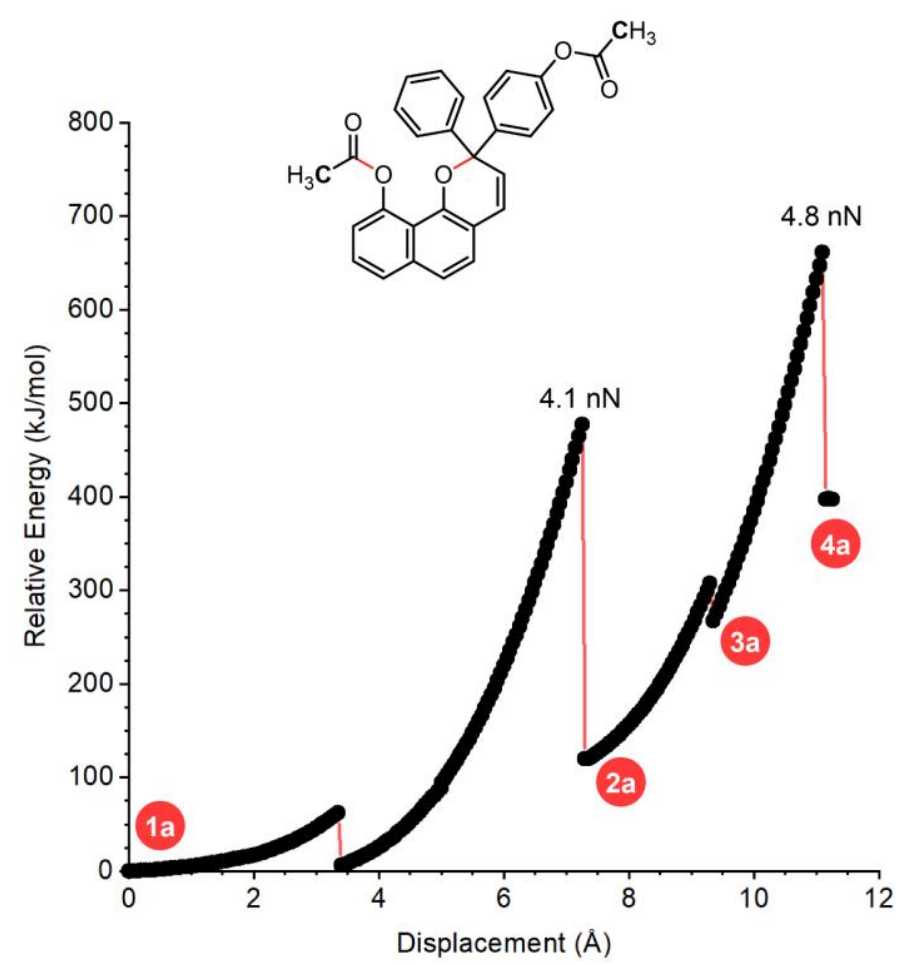

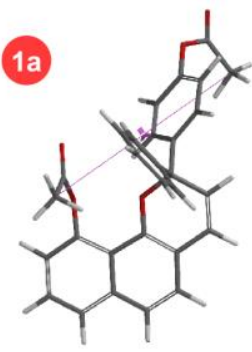

$9.581 \AA$

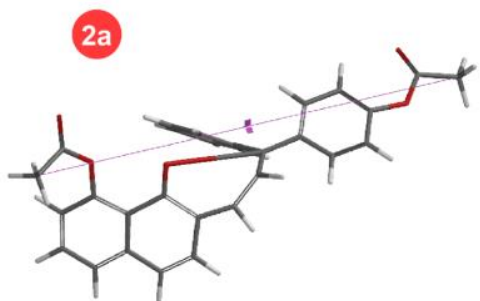

$16.931 \AA$

3 a

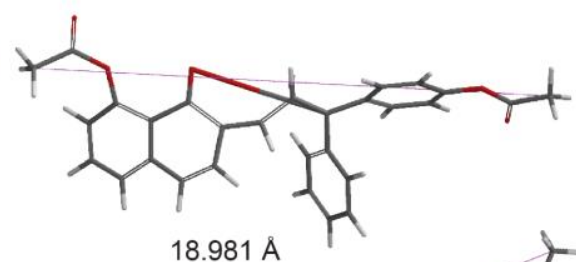

$18.981 \AA$

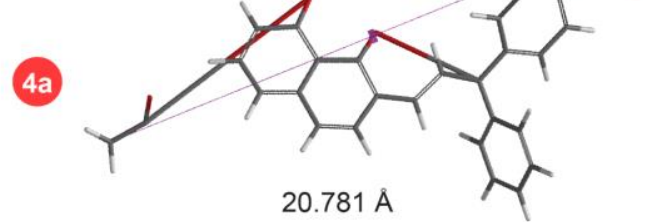

Figure S5. DFT calculations using the constrained geometries simulate external force (CoGEF) method at the B3LYP/6-31G* level of theory for a truncated model reflecting the mechanophore in polymer P1. CoGEF calculations predict the ring-opening reaction with an $F_{\max }$ value of $4.1 \mathrm{nN}$ followed by further elongation that results in $\mathrm{C}-\mathrm{O}$ bond scission at a predicted $F_{\max }$ value of $4.8 \mathrm{nN}$. The corresponding computed structures at various points of elongation are shown at right along with the associated constraint distance between the terminal methyl groups. 


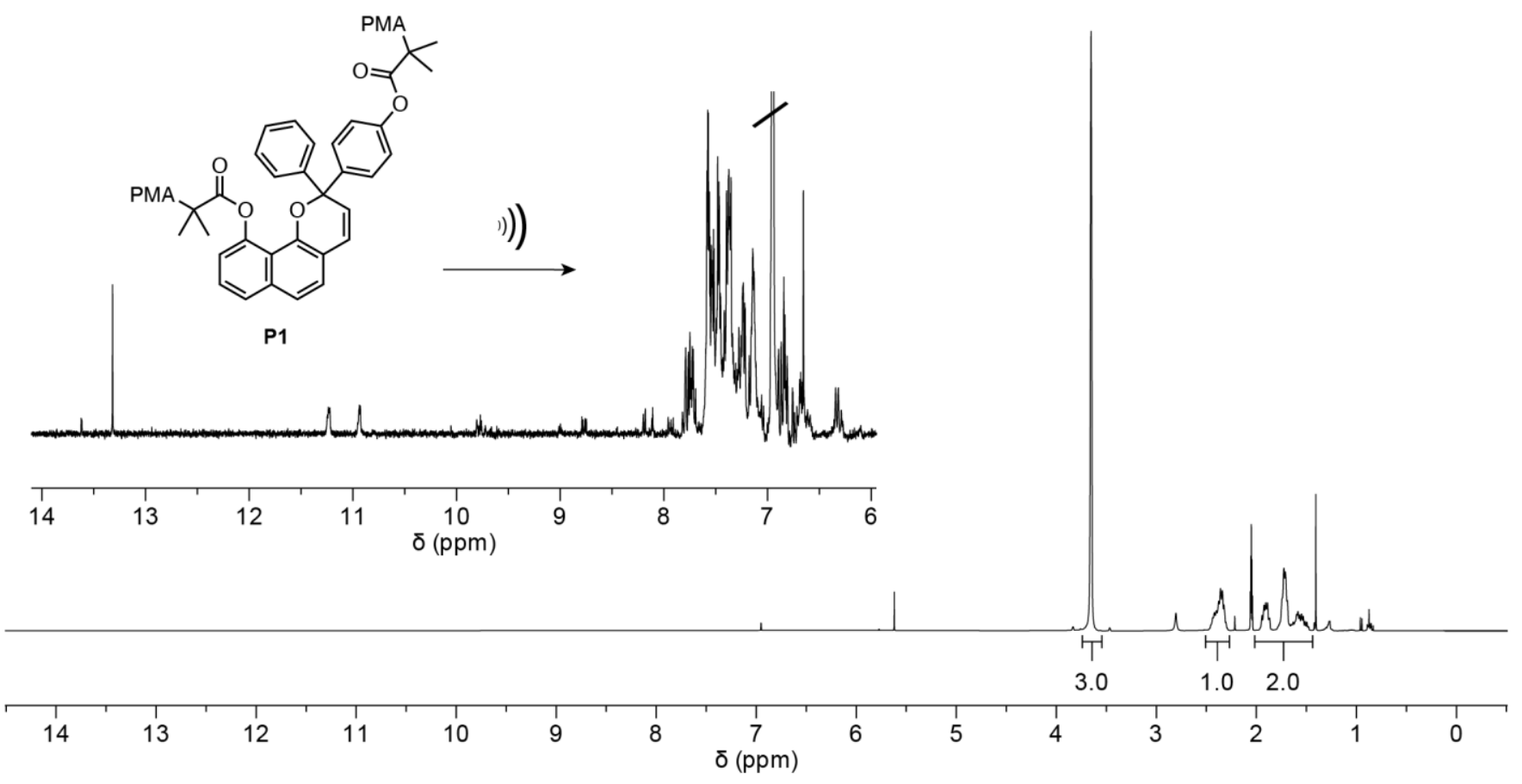

(b) $\mathbf{M 1},{ }^{1} \mathrm{H}\left(400 \mathrm{MHz}\right.$, acetone- $\left.d_{6}\right)$
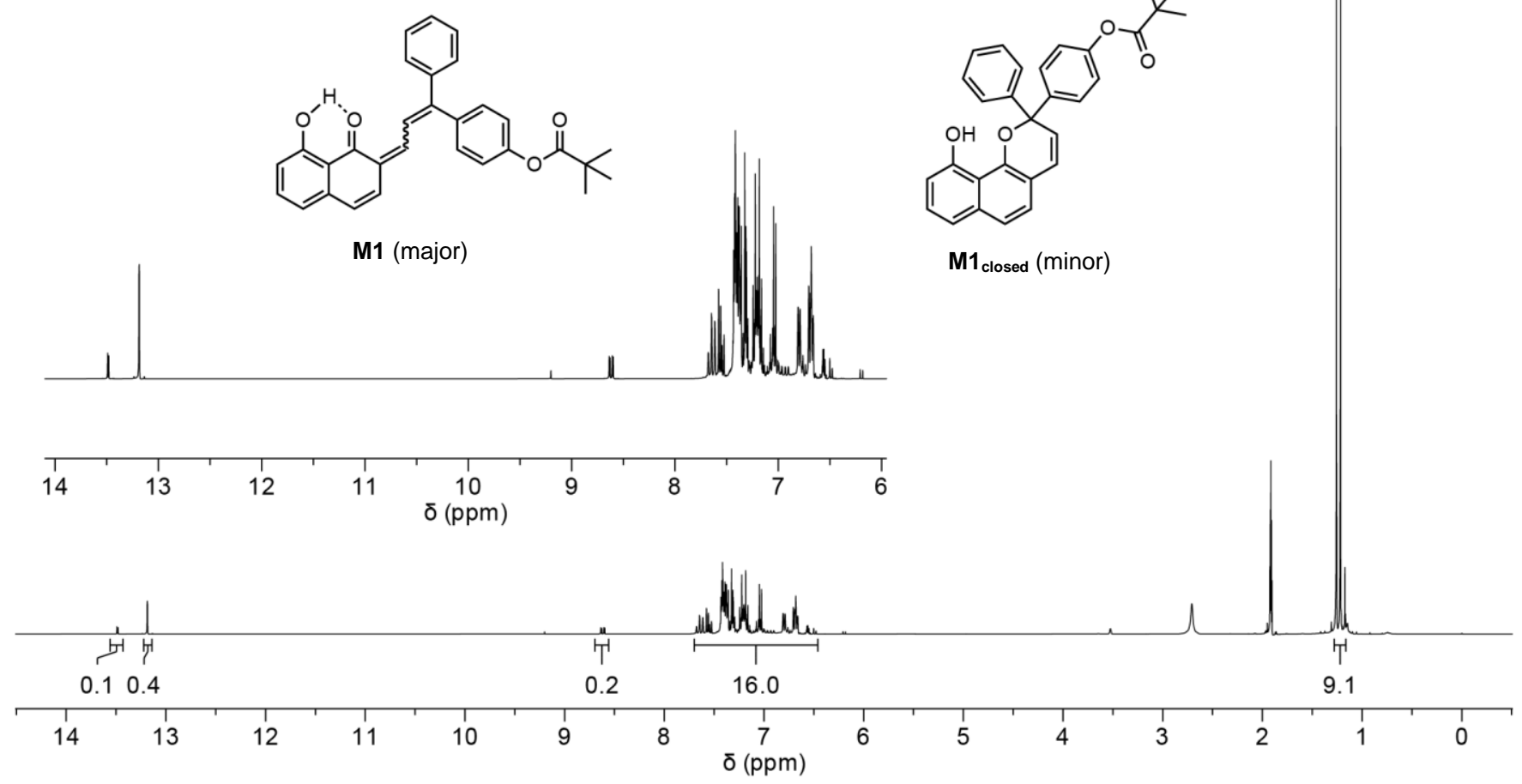

Figure S6. ${ }^{1} \mathrm{H}$ NMR spectra of (a) P1 after ultrasonication (125 min), and (b) M1. Polymer P1 was precipitated into hexanes and dried under vacuum prior to analysis. In the spectrum for P1, the peak at $6.95 \mathrm{ppm}$ is residual BHT and peaks at 10.94 and $11.23 \mathrm{ppm}$ are attributed to peroxide impurities resulting from the decomposition of THF. The small singlet at $9.34 \mathrm{ppm}$ in the spectrum of $\mathbf{M} 1$ is attributed to the significantly more shielded hydroxyl proton of the minor ring-closed naphthopyran (M1 $\left.1_{\text {closed }}\right)$. 


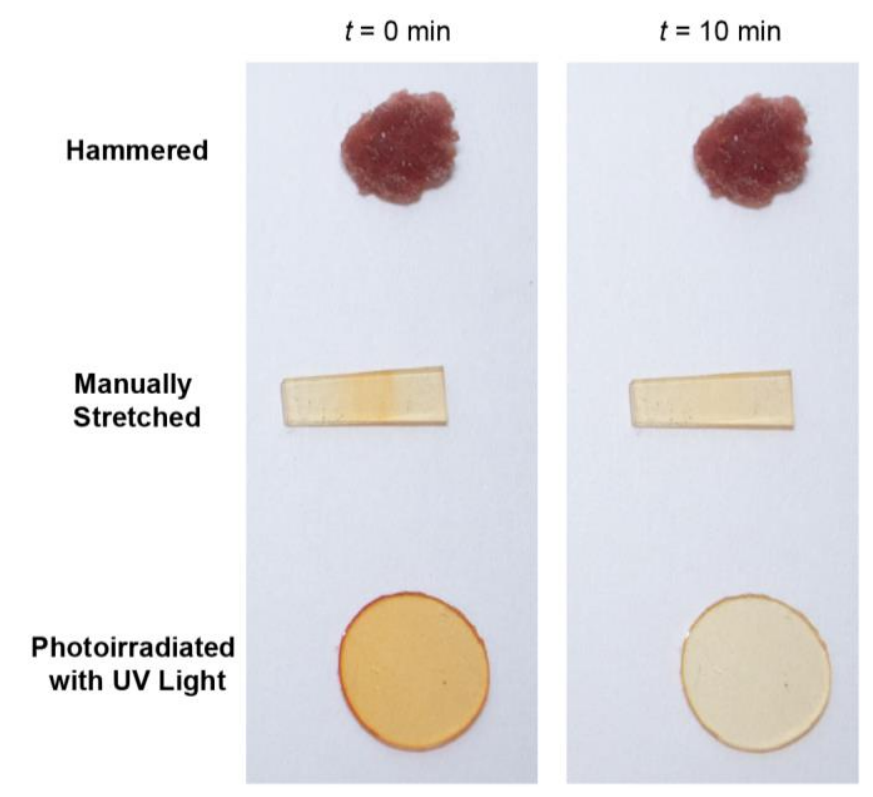

Figure S7. Photographs of PDMSactive films containing a mechanophore crosslinker after being subjected to repeated hammering, manual tension, and UV irradiation $(365 \mathrm{~nm})$ immediately after activation and then 10 min later. Manual stretching and UV light generate a transient yellow-orange color that quickly fades. In contrast, mechanical activation using a hammer generates a distinct red coloration that does not fade.

(a) Reflectance of mechanically activated PDMS $_{\text {active }}$ and controls

(b) Photographs of control experiments performed on PDMS films

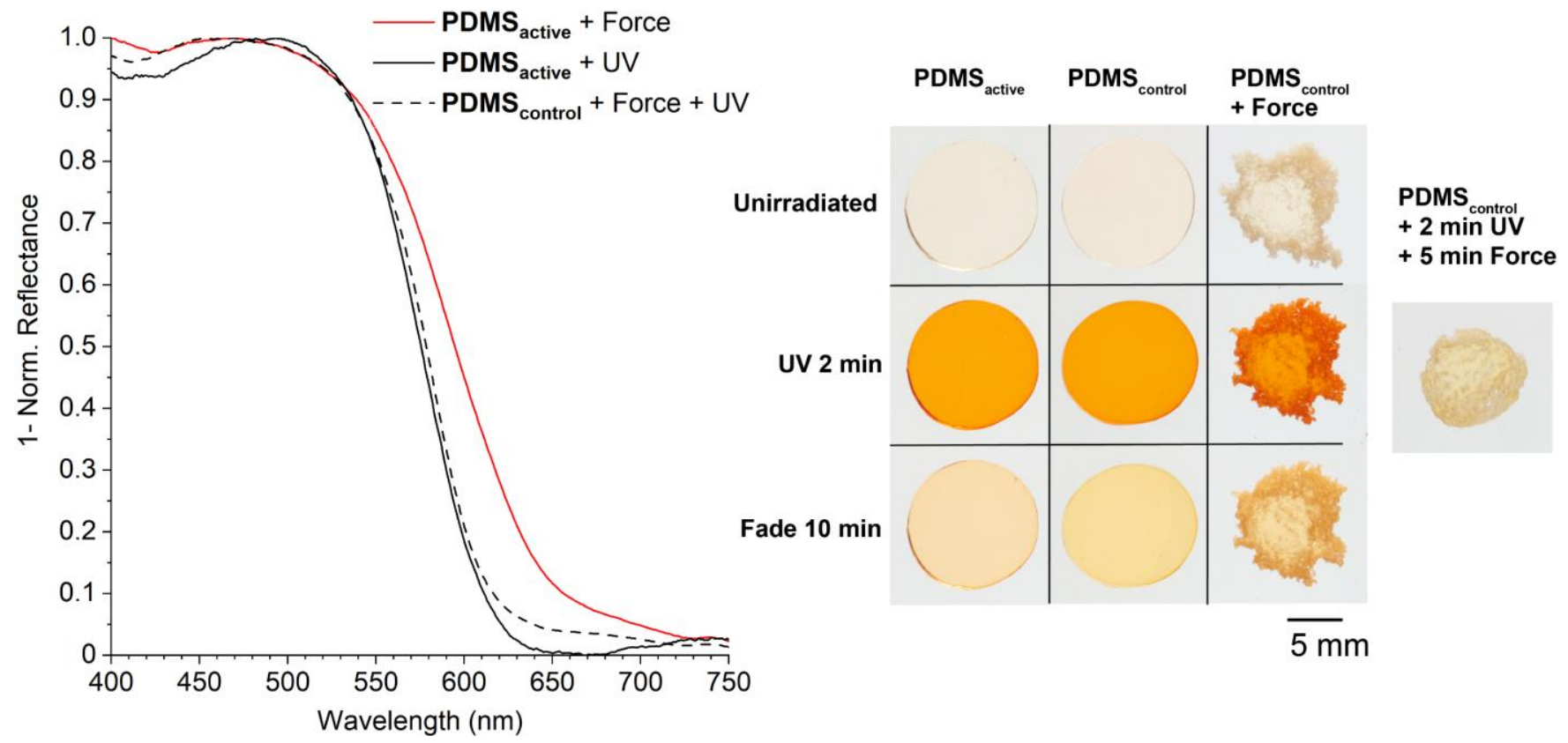

Figure S8. (a) Reflectance measurements and (b) photographs of a mechanophore-crosslinked active PDMS material (PDMS active) and a control PDMS network incorporating a mono-functional naphthopyran moiety (PDMS control) after being subjected to combinations of mechanical force by repeated hammering and UV light (365 nm). The control material exhibits similar reflection after being subjected to mechanical force and UV light as PDMS active after UV irradiation, in contrast to the bathochromically shifted reflection observed for PDMS active following mechanical activation. The control film turns yelloworange under UV light and the color quickly fades in all cases. Fading is accelerated in the photoactivated control film after being hammered for $5 \mathrm{~min}$. 


\section{Synthetic Details}

Scheme S1. Synthesis of all compounds used in this study.

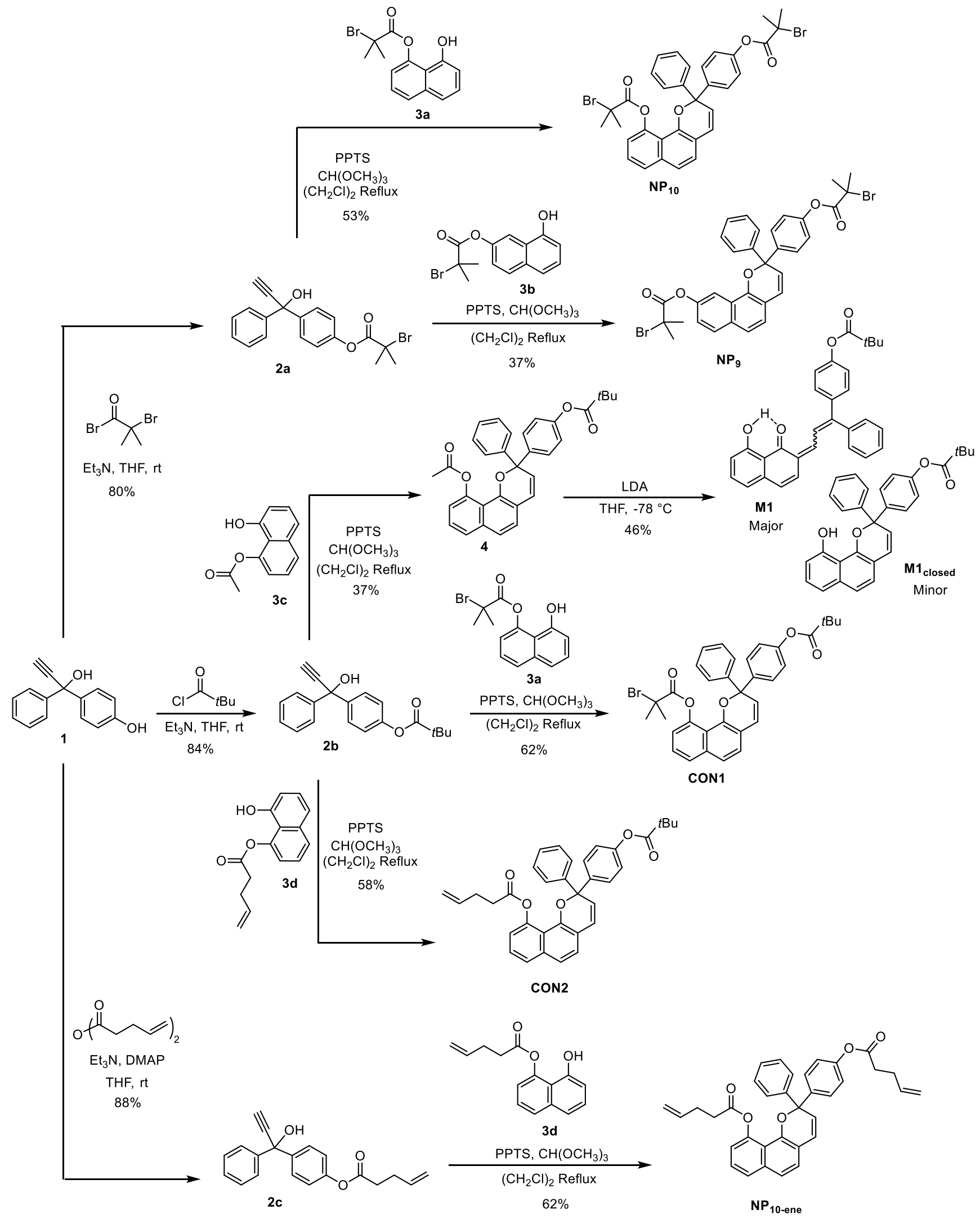




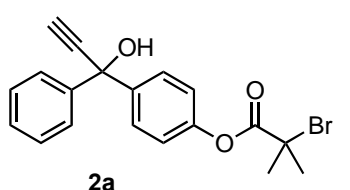

$2 a$

4-(1-hydroxy-1-phenylprop-2-yn-1-yl)phenyl 2-bromo-2-methylpropanoate (2a). To a flame-dried 2-neck $50 \mathrm{~mL}$ RBF equipped with a stir bar was added 1 (773 mg, $3.45 \mathrm{mmol}$ ).

The flask was evacuated and backfilled with $\mathrm{N}_{2}$ three times. Anhydrous THF $(20 \mathrm{~mL}$ ) and then triethylamine $(0.65 \mathrm{~mL}, 4.7 \mathrm{mmol})$ were added via syringe under $\mathrm{N}_{2} . \alpha$ bromoisobutyryl bromide $\left(0.42 \mathrm{~mL}, 3.4 \mathrm{mmol}\right.$ ) was added dropwise via syringe over 10 min under $\mathrm{N}_{2}$ at room temperature. After stirring for $19 \mathrm{~h}$, the reaction was diluted with EtOAc $(100 \mathrm{~mL})$, washed with DI water $(100 \mathrm{~mL})$, saturated aqueous $\mathrm{NaHCO}_{3}(100 \mathrm{~mL})$, and brine $(50 \mathrm{~mL})$. The organic layer was dried over $\mathrm{MgSO}_{4}$, filtered, and concentrated. The crude yellow oil was purified by column chromatography on silica gel (5-30\% EtOAc/hexanes) to yield the product as a pale yellow oil that solidified upon trituration with hexanes $(1.01 \mathrm{~g}, 78 \%)$.

$\underline{\mathrm{TLC}}$ (25\% EtOAc/hexanes): $\mathrm{R}_{f}=0.57$

${ }^{1} \mathrm{H} \mathrm{NMR}\left(400 \mathrm{MHz}, \mathrm{CDCl}_{3}\right)$ 8: $7.68-7.57(\mathrm{~m}, 4 \mathrm{H}), 7.38-7.26(\mathrm{~m}, 3 \mathrm{H}), 7.13-7.07(\mathrm{~m}, 2 \mathrm{H}), 2.89(\mathrm{~s}, 1 \mathrm{H}), 2.86(\mathrm{~s}, 1 \mathrm{H})$, $2.06(\mathrm{~s}, 6 \mathrm{H})$.

${ }^{13} \mathrm{C}\left\{{ }^{1} \mathrm{H}\right\}$ NMR $\left(101 \mathrm{MHz}, \mathrm{CDCl}_{3}\right) \delta: 170.3,150.4,144.2,142.5,128.5,128.2,127.5,126.1,120.9,86.2,76.0,74.0$, $55.4,30.7$.

HRMS (FAB, $m / z)$ : calcd for $\left[\mathrm{C}_{19} \mathrm{H}_{17} \mathrm{BrO}_{3}\right]^{+}(\mathrm{M})^{+}, 372.0361$; found, 372.0372 .

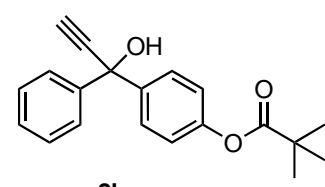

$2 b$

4-(1-hydroxy-1-phenylprop-2-yn-1-yl)phenyl pivalate (2b). To a flame-dried 2-neck 100 $\mathrm{mL}$ RBF equipped with a stir bar was added 1 (922 mg, $4.11 \mathrm{mmol})$. The flask was evacuated and backfilled with $\mathrm{N}_{2}$ three times. Anhydrous THF $(30 \mathrm{~mL})$ and subsequently triethylamine $(0.80 \mathrm{~mL}, 5.7 \mathrm{mmol})$ was added via syringe under $\mathrm{N}_{2}$. Pivaloyl chloride $(0.58$ $\mathrm{mL}, 4.7 \mathrm{mmol}$ ) was added dropwise via syringe over $10 \mathrm{~min}$ at room temperature. After stirring for $20 \mathrm{~h}$, the reaction was diluted with $100 \mathrm{~mL}$ EtOAc, washed with $10 \%$ aqueous $\mathrm{NH}_{4} \mathrm{Cl}(100 \mathrm{~mL})$, saturated aqueous $\mathrm{NaHCO}_{3}$ $(100 \mathrm{~mL})$ and brine $(50 \mathrm{~mL})$. The organic layer was dried over $\mathrm{MgSO}_{4}$, filtered, and concentrated to a yellow oil. The crude material purified by column chromatography on silica gel (10-30\% EtOAc/hexanes) to yield the product as a pale yellow solid (1.06 g, 83\%).

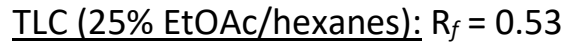

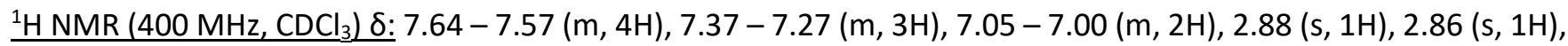
$1.35(\mathrm{~s}, 9 \mathrm{H})$.

${ }^{13} \mathrm{C}\left\{{ }^{1} \mathrm{H}\right\} \mathrm{NMR}\left(101 \mathrm{MHz}, \mathrm{CDCl}_{3}\right)$ 8: $177.1,150.8,144.3,141.8,128.5,128.1,127.3,126.1,121.4,86.3,75.8,74.1$, 39.2, 27.2.

$\underline{\mathrm{HRMS}}(\mathrm{FAB}, \mathrm{m} / \mathrm{z})$ : calcd for $\left[\underline{\mathrm{C}}_{20} \underline{\mathrm{H}}_{20} \underline{\mathrm{O}_{3}}\right]^{+}(\mathrm{M})^{+}, 309.1491$; found, 309.1471 .

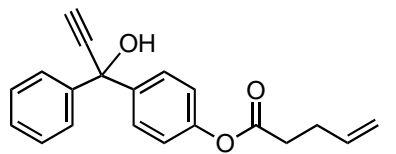

2c

4-(1-hydroxy-1-phenylprop-2-yn-1-yl)phenyl pent-4-enoate (2c). To a flame-dried 2neck $50 \mathrm{~mL}$ RBF equipped with a stir bar was added 1 (307 mg, $1.37 \mathrm{mmol}$ ) and catalytic $\mathrm{N}, \mathrm{N}$-dimethylaminopyridine $(10 \mathrm{mg}, 0.08 \mathrm{mmol})$. The flask was evacuated and 
backfilled with $\mathrm{N}_{2}$ three times. Anhydrous THF $(20 \mathrm{~mL})$ and subsequently triethylamine $(0.23 \mathrm{~mL}, 1.6 \mathrm{mmol})$ was added via syringe under $\mathrm{N}_{2}$. Pentenoic anhydride $(0.28 \mathrm{~mL}, 1.5 \mathrm{mmol})$ was added dropwise via syringe over $10 \mathrm{~min}$ room temperature. After stirring for $22 \mathrm{~h}$, the reaction was diluted with $100 \mathrm{~mL}$ EtOAc, washed with DI water (50 $\mathrm{mL})$, saturated aqueous $\mathrm{NaHCO}_{3}(50 \mathrm{~mL})$ and brine $(30 \mathrm{~mL})$. The organic layer was dried over $\mathrm{MgSO}_{4}$, filtered, and concentrated. The crude material purified by column chromatography on silica gel (0-30\% EtOAc/hexanes) to yield the product as a pale yellow oil ( $371 \mathrm{mg}, 88 \%)$.

$\underline{\operatorname{TLC}(25 \% \text { EtOAc/hexanes): }} \mathrm{R}_{f}=0.51$

${ }^{1} \mathrm{H}$ NMR $\left(400 \mathrm{MHz}\right.$, Acetone- $\left.d_{6}\right) \delta: 7.69-7.62(\mathrm{~m}, 4 \mathrm{H}), 7.37-7.30(\mathrm{~m}, 2 \mathrm{H}), 7.28-7.22(\mathrm{~m}, 1 \mathrm{H}), 7.10-7.05(\mathrm{~m}$, $2 \mathrm{H}), 5.92(\mathrm{ddt}, J=16.9,10.2,6.5 \mathrm{~Hz}, 1 \mathrm{H}), 5.77(\mathrm{~s}, 1 \mathrm{H}), 5.13(\mathrm{dq}, J=17.2,1.7 \mathrm{~Hz}, 1 \mathrm{H}), 5.03(\mathrm{dq}, J=10.2,1.4 \mathrm{~Hz}, 1 \mathrm{H})$, $3.38(\mathrm{~d}, J=0.6 \mathrm{~Hz}, 1 \mathrm{H}), 2.67(\mathrm{t}, J=7.3 \mathrm{~Hz}, 2 \mathrm{H}), 2.50-2.39(\mathrm{~m}, 2 \mathrm{H})$.

${ }^{13} \mathrm{C}\left\{{ }^{1} \mathrm{H}\right\}$ NMR $\left(101 \mathrm{MHz}\right.$, Acetone- $\left.d_{6}\right) \delta: 171.8,151.1,146.7,144.4,137.7,128.9,128.2,127.9,126.7,122.1,116.0$, 87.8, 76.5, 74.0, 34.0.

HRMS (FAB, $m / z)$ : calcd for $\left[\mathrm{C}_{20} \mathrm{H}_{19} \mathrm{O}_{3}\right]^{+}(\mathrm{M}+\mathrm{H})^{+}, 307.1334$; found, 307.1327.
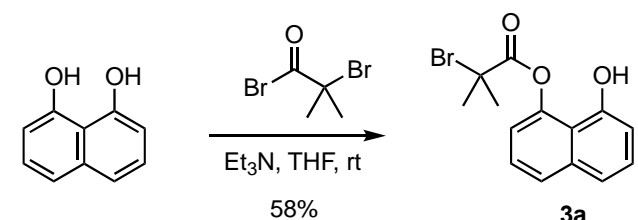

8-hydroxynaphthalen-1-yl 2-bromo-2-methylpropanoate (3a). To a flame-dried 2-neck $100 \mathrm{~mL}$ RBF equipped with a stir bar was added 1,8-dihydroxynaphthalene $(2.31 \mathrm{~g}, 14.4 \mathrm{mmol})$. The flask was evacuated and backfilled with $\mathrm{N}_{2}$ three times. Anhydrous THF $(40 \mathrm{~mL})$ and then triethylamine $(2.60 \mathrm{~mL}, 18.6 \mathrm{mmol})$ were added via syringe under $\mathrm{N}_{2}$, followed by the dropwise addition of $\alpha$-bromoisobutyryl bromide ( $2.00 \mathrm{~mL}, 16.3 \mathrm{mmol}$ ) via syringe over $10 \mathrm{~min}$ at room temperature. After stirring for $19 \mathrm{~h}$, the reaction was diluted with $100 \mathrm{~mL}$ EtOAc, washed with aqueous $10 \% \mathrm{NH}_{4} \mathrm{Cl}(2 \times 100 \mathrm{~mL})$, saturated aqueous $\mathrm{NaHCO}_{3}(100 \mathrm{~mL})$, and brine $(50 \mathrm{~mL})$. The organic layer was dried over $\mathrm{MgSO}_{4}$, filtered, and concentrated. The crude material was dissolved in hot toluene $(50 \mathrm{~mL})$ and recrystallized in the dark to yield the product as grey crystals $(2.57 \mathrm{~g}, 58 \%)$.

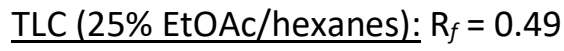

$\underline{{ }^{1} \mathrm{H} N M R}\left(400 \mathrm{MHz}, \mathrm{CDCl}_{3}\right) \delta: 7.70(\mathrm{dd}, J=8.3,1.1 \mathrm{~Hz}, 1 \mathrm{H}), 7.44-7.38(\mathrm{~m}, 2 \mathrm{H}), 7.32(\mathrm{dd}, J=8.3,7.6 \mathrm{~Hz}, 1 \mathrm{H}), 7.17$ (dd, $J=7.6,1.1 \mathrm{~Hz}, 1 \mathrm{H}), 7.06(\mathrm{~s}, 1 \mathrm{H}), 6.85(\mathrm{dd}, J=7.6,1.1 \mathrm{~Hz}, 1 \mathrm{H}), 2.43(\mathrm{~s}, 3 \mathrm{H})$.

${ }^{13} \mathrm{C}\left\{{ }^{1} \mathrm{H}\right\}$ NMR $\left(101 \mathrm{MHz}^{\mathrm{COCCl}} 3\right) \delta: 169.8,151.6,146.3,137.0,127.3,127.0,125.5,120.6,117.8,117.0,111.8,55.5$, 31.0 .

HRMS (ESI, $m / z)$ : calcd for $\left[\mathrm{C}_{14} \mathrm{H}_{13} \mathrm{BrO}_{3}\right]^{+}(\mathrm{M}+\mathrm{H})^{+}, 309.0126$; found, 309.0151 . 

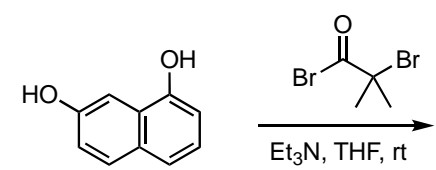

$6 \%$

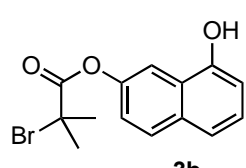

3b

8-hydroxynaphthalen-2-yl 2-bromo-2-methylpropanoate (3b). To a flame-dried 2-neck $100 \mathrm{~mL}$ RBF equipped with a stir bar was added 1,7-dihydroxynaphthalene $(2.99 \mathrm{~g}, 18.7 \mathrm{mmol})$. The flask was evacuated and backfilled with $\mathrm{N}_{2}$ three times. Anhydrous THF $(60 \mathrm{~mL})$ and subsequently triethylamine $(3.40 \mathrm{~mL}, 24.4 \mathrm{mmol})$ was added via syringe under $\mathrm{N}_{2}$. $\alpha$-bromoisobutyryl bromide $(2.25 \mathrm{~mL}, 18.5 \mathrm{mmol}$ ) was added dropwise via syringe over $10 \mathrm{~min}$ under $\mathrm{N}_{2}$ at room temperature. After stirring for $17 \mathrm{~h}$, the reaction was diluted with $150 \mathrm{~mL}$ EtOAc, washed with DI water (100 mL, aqueous layer turns pink), saturated aqueous $\mathrm{NaHCO}_{3}(100 \mathrm{~mL})$, and brine $(50 \mathrm{~mL})$. The organic layer was dried over $\mathrm{MgSO}_{4}$, filtered, and concentrated onto celite. The material was partially purified by column chromatography on silica gel (10-100\% EtOAc/hexanes), then product-containing fractions were loaded onto a plug of basic alumina and washed with $25 \% \mathrm{EtOAc/hex}$ to remove diester side product, and lastly the product and its constitutional isomer were eluted with $5 \% \mathrm{MeOH} / \mathrm{CH}_{2} \mathrm{Cl}_{2}$. A second silica column was run $(10-50 \%$ EtOAc/hexanes with $1 \%$ triethylamine) and then a third (25-100\% 10-50\% EtOAc/hexanes with 1\% triethylamine) to finally yield the pure product as a grey solid (341 $\mathrm{mg}, 6 \%)$.

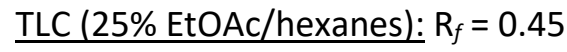

${ }^{1} \mathrm{H}$ NMR $\left(400 \mathrm{MHz}\right.$, Acetone- $\left.\mathrm{d}_{6}\right) \delta: 9.20$ (br s, $\left.1 \mathrm{H}\right), 7.99(\mathrm{~d}, J=2.5 \mathrm{~Hz}, 1 \mathrm{H}), 7.92(\mathrm{~d}, J=8.8 \mathrm{~Hz}, 1 \mathrm{H}), 7.44(\mathrm{~d}, J=8.3$ $\mathrm{Hz}, 1 \mathrm{H}), 7.34(\mathrm{~d}, J=7.5 \mathrm{~Hz}, 1 \mathrm{H}), 7.31(\mathrm{dd}, J=8.8,2.5 \mathrm{~Hz}, 1 \mathrm{H}), 6.99(\mathrm{dd}, J=7.5,1.0 \mathrm{~Hz}, 1 \mathrm{H}), 2.13(\mathrm{~s}, 6 \mathrm{H})$.

${ }^{13} \mathrm{C}\left\{{ }^{1} \mathrm{H}\right\} \mathrm{NMR}\left(101 \mathrm{MHz}\right.$, Acetone- $\left.d_{6}\right) \delta: 170.9,153.7,148.8,133.9,130.0,127.2,125.9,121.7,119.8,113.8$, 109.6, 57.2, 30.8.

HRMS (FAB, $m / z)$ : calcd for $\left[\mathrm{C}_{14} \mathrm{H}_{13} \mathrm{BrO}_{3}\right]^{+}(\mathrm{M})^{+}, 308.0048$; found, 308.0076 .

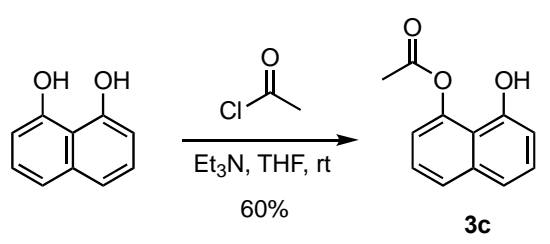

8-hydroxynaphthalen-1-yl acetate (3c). To a flame-dried 2-neck $50 \mathrm{~mL}$ RBF equipped with a stir bar was added 1,8-dihydroxynaphthalene $(1.01 \mathrm{~g}, 6.30 \mathrm{mmol})$. The flask was evacuated and backfilled with $\mathrm{N}_{2}$ three times. Anhydrous THF $(20 \mathrm{~mL})$ and subsequently triethylamine $(1.10 \mathrm{~mL}, 7.89 \mathrm{mmol})$ was added via syringe under $\mathrm{N}_{2}$. Acetyl chloride $(0.50 \mathrm{~mL}, 7.0 \mathrm{mmol})$ was added dropwise via syringe over $10 \mathrm{~min}$ at room temperature. After stirring for $3.5 \mathrm{~h}$, the reaction was diluted with $100 \mathrm{~mL}$ EtOAc, washed with DI water $(50 \mathrm{~mL}), 10 \%$ aqueous $\mathrm{NH}_{4} \mathrm{Cl}$ $(50 \mathrm{~mL})$, and brine $(50 \mathrm{~mL})$. The organic layer was dried over $\mathrm{MgSO}_{4}$, filtered, and concentrated. The crude material was recrystallized from toluene in the dark to yield the product as beige crystals $(0.76 \mathrm{~g}, 60 \%)$.

$\underline{\operatorname{TLC}(25 \% \text { EtOAc/hexanes): }} \mathrm{R}_{f}=0.26$

${ }^{1} \mathrm{H} \mathrm{NMR}\left(400 \mathrm{MHz} \mathrm{CDCl}_{3}\right) \delta: 7.70(\mathrm{dd}, J=8.3,1.1 \mathrm{~Hz}, 1 \mathrm{H}), 7.44-7.38(\mathrm{~m}, 2 \mathrm{H}), 7.32(\mathrm{dd}, J=8.3,7.6 \mathrm{~Hz}, 1 \mathrm{H}), 7.17$ (dd, $J=7.6,1.1 \mathrm{~Hz}, 1 \mathrm{H}), 7.06(\mathrm{~s}, 1 \mathrm{H}), 6.85$ (dd, $J=7.6,1.1 \mathrm{~Hz}, 1 \mathrm{H}), 2.43(\mathrm{~s}, 3 \mathrm{H})$. 

HRMS (FAB, $m / z)$ : calcd for $\left[\mathrm{C}_{12} \mathrm{H}_{10} \mathrm{O}_{3}\right]^{+}(\mathrm{M})^{+}, 202.0630$; found, 202.0638 .

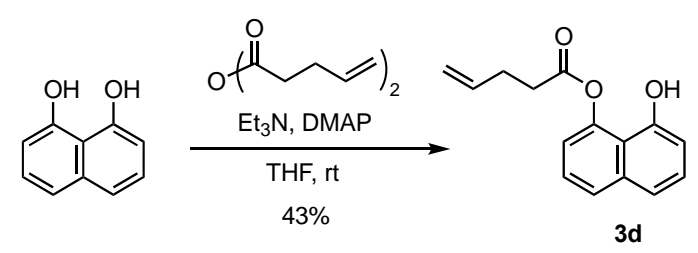

8-hydroxynaphthalen-1-yl pent-4-enoate (3d). To a flame-dried 2-neck $50 \mathrm{~mL} \mathrm{RBF}$ equipped with a stir bar was added 1,8-dihydroxynaphthalene $(687 \mathrm{mg}, 4.29 \mathrm{mmol}$ ) and catalytic $\mathrm{N}, \mathrm{N}$-dimethylaminopyridine $(5.5 \mathrm{mg}, 0.045$ $\mathrm{mmol})$. The flask was evacuated and backfilled with $\mathrm{N}_{2}$ three times. Anhydrous THF $(15 \mathrm{~mL})$ and subsequently triethylamine $(0.72 \mathrm{~mL}, 5.2 \mathrm{mmol})$ was added via syringe under $\mathrm{N}_{2}$. Pentenoic anhydride $(0.89 \mathrm{~mL}, 4.7 \mathrm{mmol})$ was added dropwise via syringe over $10 \mathrm{~min}$ room temperature. After stirring for $15 \mathrm{~h}$, the reaction was diluted with $100 \mathrm{~mL}$ EtOAc, washed with DI water $(50 \mathrm{~mL})$, saturated aqueous $\mathrm{NaHCO}_{3}(50 \mathrm{~mL})$ and brine $(30 \mathrm{~mL})$. The organic layer was dried over $\mathrm{MgSO}_{4}$, filtered, and concentrated. The crude material purified by column chromatography on silica gel (5-30\% EtOAc/hexanes) and subsequently recrystallized from toluene to yield the product as colorless crystals (444 mg, 43\%).

$\underline{\mathrm{TLC}(25 \% \mathrm{EtOAC} / \text { hexanes): }} \mathrm{R}_{f}=0.56$

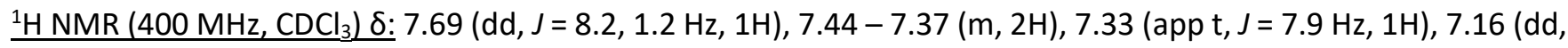
$J=7.6,1.2 \mathrm{~Hz}, 1 \mathrm{H}), 7.11(\mathrm{~d}, J=2.1 \mathrm{~Hz}, 1 \mathrm{H}), 6.86(\mathrm{dd}, J=7.6,1.2 \mathrm{~Hz}, 1 \mathrm{H}), 5.93(\mathrm{ddt}, J=16.9,10.1,6.4 \mathrm{~Hz}, 1 \mathrm{H}), 5.19$ (dq, $J=17.2,1.5 \mathrm{~Hz}, 1 \mathrm{H}), 5.12(\mathrm{dq}, J=10.2,1.4 \mathrm{~Hz}, 1 \mathrm{H}), 2.87-2.77(\mathrm{~m}, 2 \mathrm{H}), 2.63-2.53(\mathrm{~m}, 2 \mathrm{H})$.

${ }^{13} \mathrm{C}\left\{{ }^{1} \mathrm{H}\right\} \operatorname{NMR}\left(101 \mathrm{MHz}^{\mathrm{C} C D C l}{ }_{3}\right) \delta: 170.7,151.9,146.0,137.0,136.1,127.2,126.6,125.6,120.5,118.6,117.0,116.5$, $111.5,34.1,28.7$.

HRMS (FAB, $m / z)$ : calcd for $\left[\mathrm{C}_{15} \mathrm{H}_{14} \mathrm{O}_{3}\right]^{+}(\mathrm{M})^{+}, 242.0943$; found, 242.0930 .

General Procedure A for Naphthopyran Synthesis. Naphthopyrans were synthesized following the procedure by Zhao and Carreira. ${ }^{1}$ To a flame-dried 2-neck round bottom flask equipped with a stir bar and reflux condenser was added the appropriate propargyl alcohol, naphthol, and a catalytic amount of pyridinium $p$-toluenesulfonate (PPTS). The flask was evacuated and backfilled with $\mathrm{N}_{2}$ three times followed by the sequential addition of 1,2dichloroethane and trimethyl orthoformate via syringe. The reaction was heated to reflux and stirred for the indicated amount of time. Upon completion, the reaction was cooled to room temperature, concentrated with celite, and purified by column chromatography on silica gel. 


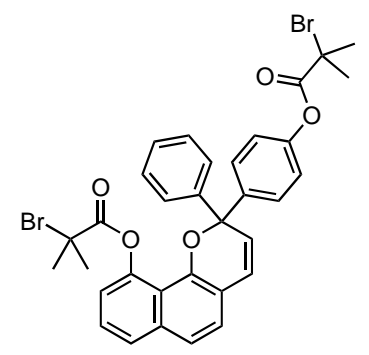

$\mathrm{NP}_{10}$ Procedure A with $3 \mathrm{a}$ ( $283 \mathrm{mg}, 0.915 \mathrm{mmol}), 2 \mathrm{a}(475 \mathrm{mg}, 1.27 \mathrm{mmol})$ added as a solution in 1,2-dichloroethane, PPTS ( $22 \mathrm{mg}, 0.088 \mathrm{mmol}$ ), trimethyl orthoformate $(0.30 \mathrm{~mL}, 2.7$ $\mathrm{mmol})$, and 1,2-dichloroethane ( $10 \mathrm{~mL}$ ) for $7 \mathrm{~h}$. Purification by column chromatography on silica gel (5-40\% EtOAc/hexanes) yielded the title compound as a pink foamy solid (322 mg, 53\%).

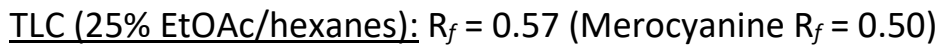

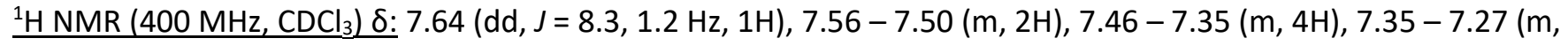
$3 \mathrm{H}), 7.17(\mathrm{dd}, J=7.5,1.2 \mathrm{~Hz}, 1 \mathrm{H}), 7.14(\mathrm{~d}, J=8.3 \mathrm{~Hz}, 1 \mathrm{H}), 7.11-7.05(\mathrm{~m}, 2 \mathrm{H}), 6.66(\mathrm{~d}, J=9.6 \mathrm{~Hz}, 1 \mathrm{H}), 6.11(\mathrm{~d}, J=$ $9.6 \mathrm{~Hz}, 1 \mathrm{H}), 2.04(\mathrm{~s}, 6 \mathrm{H}), 1.55(\mathrm{~s}, 3 \mathrm{H}), 1.54(\mathrm{~s}, 3 \mathrm{H})$.

${ }^{13} \mathrm{C}\left\{{ }^{1} \mathrm{H}\right\}$ NMR $(101 \mathrm{MHz}, \mathrm{CDCl} 33) \delta: 170.7,170.2,150.4,147.4,147.0,144.4,142.2,137.1,129.1,128.6,128.3,128.2$, $127.8,127.2,126.1,125.6,124.0,121.6,120.8,119.5,118.8,117.5,83.6,57.1,55.4,30.7,29.7$, 29.6.

HRMS (ESI, $m / z)$ : calcd for $\left[\mathrm{C}_{33} \mathrm{H}_{29} \mathrm{Br}_{2} \mathrm{O}_{5}\right]^{+}(\mathrm{M}+\mathrm{H})^{+}, 663.0382$; found, 663.0399 .

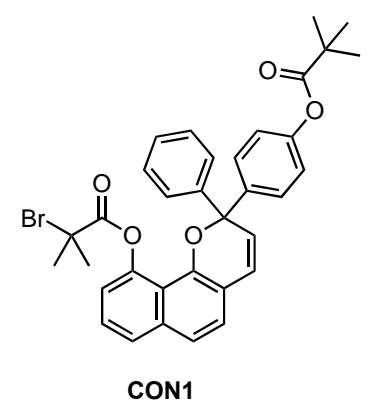

4-(10-((2-bromo-2-methylpropanoyl)oxy)-2-phenyl-2H-benzo[h]chromen-2-yl)phenyl pivalate (CON1). The title compound was prepared using General Procedure A with 3a (181 mg, $0.585 \mathrm{mmol}$ ), 2b (204 mg, $0.662 \mathrm{mmol}$ ), PPTS (8.0 mg, $0.032 \mathrm{mmol}$ ), trimethyl orthoformate $(0.20 \mathrm{~mL}, 1.8 \mathrm{mmol})$, and 1,2-dichloroethane $(9 \mathrm{~mL})$ for $17 \mathrm{~h}$. Purification by column chromatography on silica gel (10-60\% $\mathrm{CH}_{2} \mathrm{Cl}_{2} /$ hexanes) yielded the title compound as a pink foamy solid (219 $\mathrm{mg}, 62 \%)$.

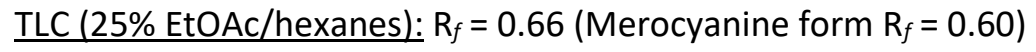

${ }^{1} \mathrm{H} \mathrm{NMR}\left(400 \mathrm{MHz}, \mathrm{CDCl}_{3}\right) \delta: 7.64(\mathrm{dd}, J=8.3,1.2 \mathrm{~Hz}, 1 \mathrm{H}), 7.53-7.48(\mathrm{~m}, 2 \mathrm{H}), 7.47-7.35(\mathrm{~m}, 4 \mathrm{H}), 7.35-7.27$ $(\mathrm{m}, 3 \mathrm{H}), 7.18(\mathrm{dd}, J=7.5,1.2 \mathrm{~Hz}, 1 \mathrm{H}), 7.13(\mathrm{~d}, J=8.3 \mathrm{~Hz}, 1 \mathrm{H}), 7.04-6.98(\mathrm{~m}, 2 \mathrm{H}), 6.65(\mathrm{~d}, J=9.6 \mathrm{~Hz}, 1 \mathrm{H}), 6.11(\mathrm{~d}$, $J=9.6 \mathrm{~Hz}, 1 \mathrm{H}), 1.56(\mathrm{~s}, 3 \mathrm{H}), 1.54(\mathrm{~s}, 3 \mathrm{H}), 1.34(\mathrm{~s}, 9 \mathrm{H})$.

${ }^{13} \mathrm{C}\left\{{ }^{1} \mathrm{H}\right\} \mathrm{NMR}\left(101 \mathrm{MHz}, \mathrm{CDCl}_{3}\right) \delta: 177.1,170.7,150.8,147.4,147.0,144.5,141.6,137.1,128.9,128.7,128.2,128.1$, $127.9,127.2,126.0,125.5,123.8,121.6,121.2,119.5,118.8,117.5,83.6,57.1,39.2,29.7,29.6,27.2$.

HRMS (ESI, $m / z):$ calcd for $\left[\mathrm{C}_{34} \mathrm{H}_{32} \mathrm{BrO}_{5}\right]^{+}(\mathrm{M}+\mathrm{H})^{+}, 599.1433$; found, 599.1441.

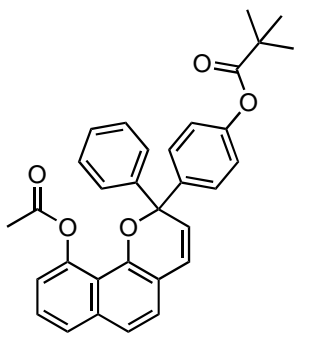

4

4-(10-acetoxy-2-phenyl-2H-benzo[h]chromen-2-yl)phenyl pivalate (4). The title compound was prepared using General Procedure A with 3c (519 mg, $2.57 \mathrm{mmol}$ ), 2b (905 mg, 2.93 $\mathrm{mmol})$, PPTS (32.7 $\mathrm{mg}, 0.130 \mathrm{mmol})$, trimethyl orthoformate $(0.70 \mathrm{~mL}, 6.4 \mathrm{mmol})$, and 1,2dichloroethane $(20 \mathrm{~mL})$ for $19 \mathrm{~h}$. The crude reaction mixture was filtered over basic alumina, eluted with EtOAc, and purification by column chromatography on silica gel (0-25\% EtOAc/hexanes) yielded the title compound as pink foamy solid (466 mg, 37\%). 


\section{ILC (25\% EtOAc/hexanes): $\mathrm{R}_{f}=0.47$}

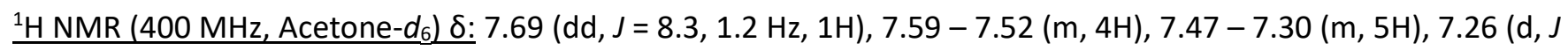
$=8.3 \mathrm{~Hz}, 1 \mathrm{H}), 7.14-7.08(\mathrm{~m}, 3 \mathrm{H}), 6.83(\mathrm{~d}, J=9.7 \mathrm{~Hz}, 1 \mathrm{H}), 6.34(\mathrm{~d}, J=9.7 \mathrm{~Hz}, 1 \mathrm{H}), 1.68(\mathrm{~s}, 3 \mathrm{H}), 1.32(\mathrm{~s}, 9 \mathrm{H})$.

${ }^{13} \mathrm{C}\left\{{ }^{1} \mathrm{H}\right\}$ NMR $\left(101 \mathrm{MHz}\right.$, Acetone- $\left.d_{6}\right) \delta:$ 177.2, 170.4, 152.0, 148.1, 147.7, 145.5, 142.9, 138.0, 129.7, 129.4, 129.0, $128.9,128.4,127.3,127.1,126.3,124.7,122.24,122.22,121.1,120.2,118.3,84.5,39.7,27.4,20.8$.

HRMS (ESI, $m / z)$ : calcd for $\left[\mathrm{C}_{32} \mathrm{H}_{29} \mathrm{O}_{5}\right]^{+}(\mathrm{M}+\mathrm{H})^{+}, 493.2015$; found, 493.2005.

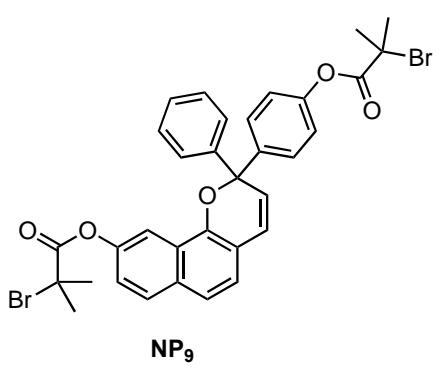

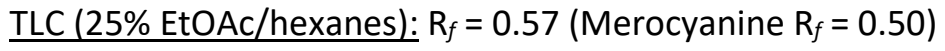
$4 \mathrm{H}), 7.23(\mathrm{dd}, J=8.9,2.4 \mathrm{~Hz}, 1 \mathrm{H}), 7.16(\mathrm{~d}, J=8.4 \mathrm{~Hz}, 1 \mathrm{H}), 7.14-7.08(\mathrm{~m}, 2 \mathrm{H}), 6.75(\mathrm{~d}, J=9.8 \mathrm{~Hz}, 1 \mathrm{H}), 6.17(\mathrm{~d}, J=$ $9.7 \mathrm{~Hz}, 1 \mathrm{H}), 2.13(\mathrm{~s}, 6 \mathrm{H}), 2.04(\mathrm{~s}, 6 \mathrm{H})$.

${ }^{13} \mathrm{C}\left\{{ }^{1} \mathrm{H}\right\}$ NMR $(101 \mathrm{MHz}, \mathrm{CDCl} 33) \delta: 170.5,170.3,150.2,148.7,147.5,144.7,142.8,132.9,129.5,128.5,128.4,127.9$, $127.8,127.1,125.0,124.7,123.9,121.0,120.8,120.7,116.2,112.7,83.3,55.7,55.4,30.9,30.7$.

HRMS (ESI, $m / z)$ : calcd for $\left[\mathrm{C}_{33} \mathrm{H}_{29} \mathrm{Br}_{2} \mathrm{O}_{5}\right]^{+}(\mathrm{M}+\mathrm{H})^{+}, 663.0382$; found, 663.0390 .

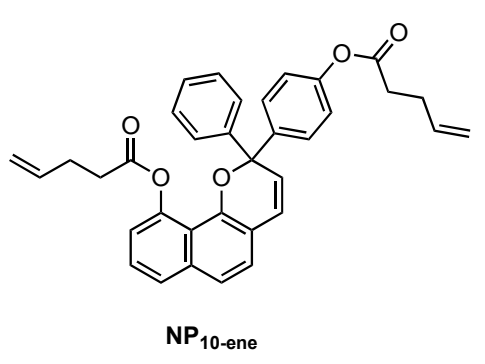

4-(10-(pent-4-enoyloxy)-2-phenyl-2H-benzo[h]chromen-2-yl)phenyl pent-4enoate ( $\mathbf{N P}_{10-e n e}$ ). The title compound was prepared using General Procedure A with $3 \mathrm{~d}$ (102 $\mathrm{mg}, 0.420 \mathrm{mmol}), 2 \mathrm{c}(148 \mathrm{mg}, 0.483 \mathrm{mmol}$ ) added as a solution in 1,2-dichloroethane, PPTS $(9.4 \mathrm{mg}, 0.037 \mathrm{mmol})$, trimethyl orthoformate $(0.14$ $\mathrm{mL}, 1.3 \mathrm{mmol})$, and 1,2-dichloroethane $(6 \mathrm{~mL})$ for $2 \mathrm{~h}$. Purification by column chromatography on silica gel (0-10\% EtOAc with $1 \%$ triethylamine/hexanes) yielded the title compound as a dark orange-red oil (138 mg, 62\%).

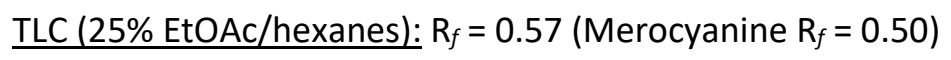

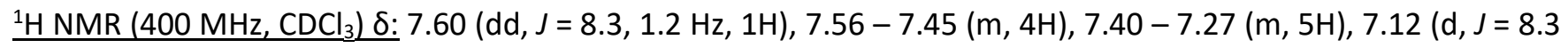
$\mathrm{Hz}, 1 \mathrm{H}), 7.08-6.98(\mathrm{~m}, 3 \mathrm{H}), 6.66(\mathrm{~d}, J=9.7 \mathrm{~Hz}, 1 \mathrm{H}), 6.12(\mathrm{~d}, J=9.6 \mathrm{~Hz}, 1 \mathrm{H}), 5.89(\mathrm{ddt}, J=16.8,10.2,6.4 \mathrm{~Hz}, 1 \mathrm{H})$, $5.70-5.57(\mathrm{~m}, 1 \mathrm{H}), 5.13(\mathrm{dq}, J=17.1,1.6 \mathrm{~Hz}, 1 \mathrm{H}), 5.07(\mathrm{dq}, J=10.3,1.4 \mathrm{~Hz}, 1 \mathrm{H}), 4.99-4.95(\mathrm{~m}, 1 \mathrm{H}), 4.93(\mathrm{dt}, J=$ 2.6, $1.7 \mathrm{~Hz}, 1 \mathrm{H}), 2.65(\mathrm{td}, J=7.3,0.8 \mathrm{~Hz}, 2 \mathrm{H}), 2.49$ (tdd, $J=7.6,6.0,1.1 \mathrm{~Hz}, 2 \mathrm{H}), 2.20-2.06(\mathrm{~m}, 2 \mathrm{H}), 2.06-1.94(\mathrm{~m}$, $2 \mathrm{H})$. 
${ }^{13} \mathrm{C}\left\{{ }^{1} \mathrm{H}\right\} \mathrm{NMR}\left(101 \mathrm{MHz}, \mathrm{CDCl}_{3}\right) \delta: 172.6,171.6,150.4,147.5,146.6,144.6,142.1,137.3,136.9,136.5,129.0,128.3$, $128.2,128.0,127.8,126.8,126.1,125.6,124.1,121.5,121.3,1220.2,119.3,117.3,116.2,115.5,83.7,33.8,33.1$, 29.1, 28.6.

HRMS (FAB, $m / z):$ calcd for $\left[\mathrm{C}_{35} \mathrm{H}_{30} \mathrm{O}_{5}\right]^{+}(\mathrm{M})^{+}, 530.2093$; found, 530.2094.

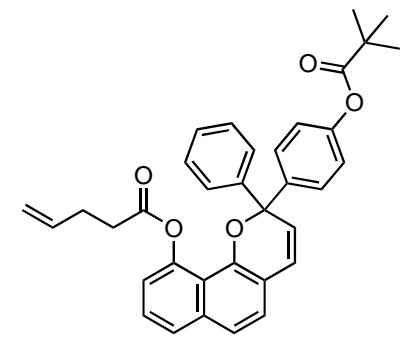

CON2

\section{2-phenyl-2-(4-(pivaloyloxy)phenyl)-2H-benzo[h]chromen-10-yl} (CON2). The title compound was prepared using General Procedure A with 3d (180 $\mathrm{mg}, 0.743 \mathrm{mmol}$ ), $\mathbf{2 b}$ (309 mg, $1.00 \mathrm{mmol}$ ) added as a solid, PPTS ( $9.6 \mathrm{mg}, 0.038 \mathrm{mmol})$, trimethyl orthoformate $(0.25 \mathrm{~mL}, 2.3 \mathrm{mmol})$, and 1,2-dichloroethane $(6 \mathrm{~mL})$ for $2 \mathrm{~h}$. Purification by column chromatography on silica gel (0-10\% EtOAc with $1 \%$ triethylamine/hexanes) yielded the title compound as a pink foamy solid (229 mg, $58 \%)$.

TLC (25\% EtOAc/hexanes): $\mathrm{R}_{f}=0.66$

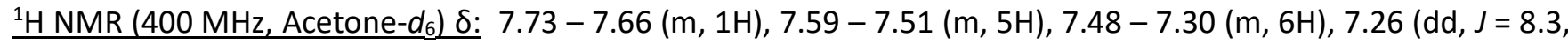
$1.0 \mathrm{~Hz}, 1 \mathrm{H}), 7.15-7.07(\mathrm{~m}, 3 \mathrm{H}), 6.83(\mathrm{dd}, J=9.7,1.1 \mathrm{~Hz}, 1 \mathrm{H}), 6.34(\mathrm{dd}, J=9.7,1.0 \mathrm{~Hz}, 1 \mathrm{H}), 5.75-5.60(\mathrm{~m}, 1 \mathrm{H})$, $5.01-4.88(\mathrm{~m}, 2 \mathrm{H}), 2.17-2.07(\mathrm{~m}, 4 \mathrm{H}), 1.32(\mathrm{~d}, J=0.7 \mathrm{~Hz}, 9 \mathrm{H})$.

${ }^{13} \mathrm{C}\left\{{ }^{1} \mathrm{H}\right\}$ NMR $\left(101 \mathrm{MHz}\right.$, Acetone- $\left.d_{6}\right) \delta: 177.1,172.5,152.0,148.2,147.7,145.5,142.8,138.0,137.9,129.7,129.6$, $129.1,129.0,128.5,127.4,127.1,126.4,124.7,122.30,122.27,121.2,120.3,118.4,115.7,84.6,39.7,33.7,29.3$, 27.4.

HRMS (FAB, $m / z)$ : calcd for $\left[\mathrm{C}_{35} \mathrm{H}_{33} \mathrm{O}_{5}\right]^{+}(\mathrm{M}+\mathrm{H})^{+}, 533.2328$; found, 533.2345 .
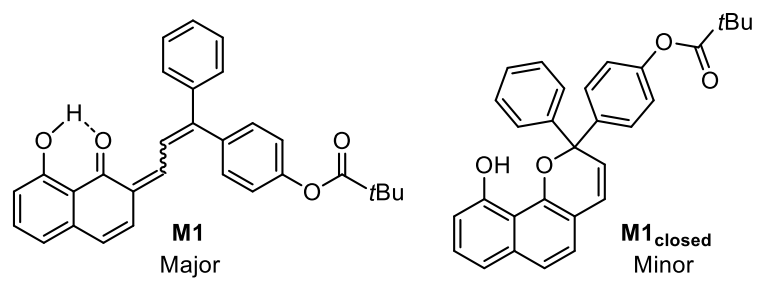

4-(3-(8-hydroxy-1-oxonaphthalen-2(1H)-ylidene)-1phenylprop-1-en-1-yl)phenyl pivalate (M1). Lithium diisopropylamine was freshly prepared in a flame-dried $25 \mathrm{~mL}$ round bottom flask. Anhydrous THF $(7 \mathrm{~mL})$ and diisopropylamine $(0.50 \mathrm{~mL}, 3.5 \mathrm{mmol})$ were added via syringe under $\mathrm{N}_{2}$. The flask was cooled to $-78^{\circ} \mathrm{C}$ and $\mathrm{n}$-butyllithium $(2.5 \mathrm{M}$ in hexanes, $1.4 \mathrm{~mL}, 3.5 \mathrm{mmol}$ ) was added via syringe under $\mathrm{N}_{2}$. The reaction was stirred for $2 \mathrm{~h}$. To a separate flame dried $100 \mathrm{~mL}$ 2-neck round bottom flask equipped with a stir bar was added $4(210 \mathrm{mg}, 0.426 \mathrm{mmol})$ and the vessel was evacuated and backfilled with $\mathrm{N}_{2}$ three times. Anhydrous THF (10 mL) was added via syringe under $\mathrm{N}_{2}$, the solution was cooled to $-78^{\circ} \mathrm{C}$, and LDA solution (1.0 mL, $0.39 \mathrm{mmol}$ ) was added dropwise via syringe, upon which the coral pink solution immediately turned yellow. After $2 \mathrm{~h}$, the reaction was removed from the cooling bath and immediately diluted with EtOAc $(100 \mathrm{~mL})$ and $10 \%$ aqueous $\mathrm{NH}_{4} \mathrm{Cl}$ solution $(120 \mathrm{~mL})$. Upon dilution, the organic layer initially becomes deep indigo in color, but the organic layer becomes a deep burgundy red color upon washing with aqueous acid. The organic layer was washed with brine $(50 \mathrm{~mL})$, dried over $\mathrm{Na}_{2} \mathrm{SO}_{4}$, filtered, and concentrated. Purification by column chromatography on silica gel (0-25\% EtOAc/hexanes) yielded the product as a red foamy solid ( $80.3 \mathrm{mg}, 46 \%)$. The product is a mixture of merocyanine stereoisomers that readily interconvert and contains $\sim 10 \%$ ring-closed 
naphthopyran as a minor product based on integrations of the hydroxyl resonances in the ${ }^{1} \mathrm{H}$ NMR spectrum measured in acetonitrile- $d_{3}$.

TLC (25\% EtOAc/hexanes): $\mathrm{R}_{f}=0.66,0.58$ (stereoisomers)

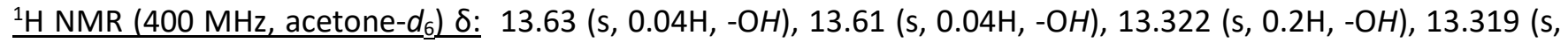
$0.2 \mathrm{H},-\mathrm{OH}), 9.34\left(\mathrm{~s}, 0.01 \mathrm{H},-\mathrm{OH}, \mathrm{M} 1_{\text {closed }}\right), 8.76(\mathrm{~d}, J=12.2 \mathrm{~Hz}, 0.09 \mathrm{H}), 8.75(\mathrm{~d}, J=12.3 \mathrm{~Hz}, 0.09 \mathrm{H}), 7.85-6.59$ (m, $16 \mathrm{H}), 6.33\left(\mathrm{~d}, J=9.9 \mathrm{~Hz}, 0.03 \mathrm{H}, \mathrm{M1}_{\text {closed }}\right) 1.40-1.30(\mathrm{~m}, 9 \mathrm{H})$.

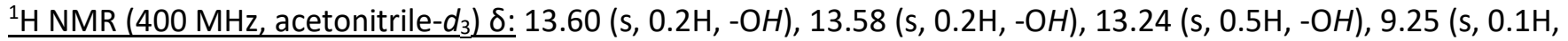
$\left.-\mathrm{OH}, \mathrm{M1}_{\text {closed }}\right), 8.67(\mathrm{~d}, J=12.1 \mathrm{~Hz}, 0.2 \mathrm{H}), 8.64(\mathrm{~d}, J=12.2 \mathrm{~Hz}, 0.2 \mathrm{H}), 7.69-6.55(\mathrm{~m}, 16 \mathrm{H}), 6.25(\mathrm{~d}, J=9.8 \mathrm{~Hz}, 0.1 \mathrm{H}$, $\left.\mathbf{M 1}_{\text {closed }}\right), 1.38-1.30(\mathrm{~m}, 9 \mathrm{H})$.

${ }^{13} \mathrm{C}\left\{{ }^{1} \mathrm{H}\right\}$ NMR $\left(101 \mathrm{MHz}\right.$, Acetone- $\left.d_{6}\right) \delta: 189.30,189.26,176.13,176.12,164.5,164.2,163.8,157.5,155.82,155.78$, $155.7,152.7,152.6,152.0,146.8,141.4,141.2,140.1,140.0,139.83,139.79,138.82,138.80,138.78,138.6,138.4$, $136.70,136.67,136.65,136.62,135.8,132.4,132.1,132.0,131.3,131.0,130.93,130.88,130.84,130.82,130.80$, $130.0,129.9,129.8,129.6,129.2,128.9,128.8,128.7,128.63,128.57,128.5,128.4,128.2,126.8,126.5,126.2$, $125.1,125.0,124.8,124.12,124.07$ 123.6, 123.54, 123.51, 122.5, 122.3, 122.1, 121.91, 121.86, 121.84, 121.79, $121.7,119.20,119.17,119.13,119.10,118.5,116.34,116.30,116.24,116.20,116.0,115.9,115.4,38.83,38.80$, $26.5,26.42,26.38$.

HRMS (ESI, $m / z):$ calcd for $\left[\mathrm{C}_{30} \mathrm{H}_{27} \mathrm{O}_{4}\right]^{+}(\mathrm{M}+\mathrm{H})^{+}, 451.1909 ;$ found, 451.1921

General Procedure B for the Synthesis of Poly(Methyl Acrylate) (PMA) Polymers Incorporating a $2 \mathrm{H}$ Naphthopyran. Polymers were synthesized by controlled radical polymerization following the procedure by Nguyen et al. ${ }^{2}$ A flame-dried Schlenk flask was charged with freshly cut $20 \mathrm{G}$ copper wire $(2 \mathrm{~cm})$, initiator, DMSO, and methyl acrylate. The flask was sealed and the solution was degassed via three freeze-pump-thaw cycles, then backfilled with nitrogen and warmed to room temperature. Me ${ }_{6}$ TREN was added via microsyringe and the reaction was stirred at room temperature for the indicated amount of time. Upon completion of the polymerization, the flask was opened to atmosphere and diluted with a minimal amount of $\mathrm{CH}_{2} \mathrm{Cl}_{2}$. The polymer was precipitated $3 x$ into methanol cooled with dry ice and then dried under vacuum to afford the polymer.
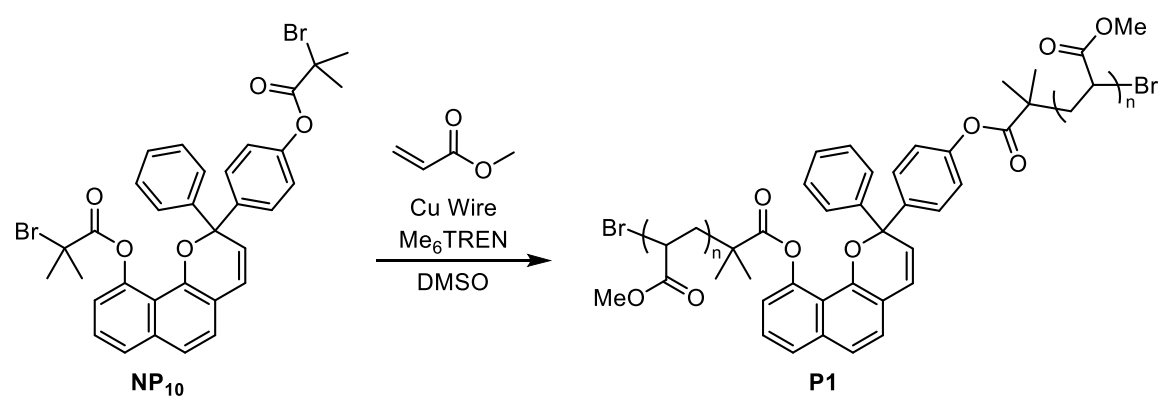

Polymer P1. Synthesized using General Procedure B with initiator $\mathbf{N P}_{10}(14.0 \mathrm{mg}, 0.021 \mathrm{mmol})$, methyl acrylate $(6.8 \mathrm{~mL}, 76 \mathrm{mmol})$, DMSO $(6.8 \mathrm{~mL})$, and $\mathrm{Me}_{6} \operatorname{TREN}(28 \mu \mathrm{L}, 0.10 \mathrm{mmol})$. Polymerization for $5 \mathrm{~h}$ provided the title polymer as a tacky orange solid $(1.6 \mathrm{~g}, 25 \%) . M_{\mathrm{n}}=178 \mathrm{~kg} / \mathrm{mol}, \emptyset=1.13$. 


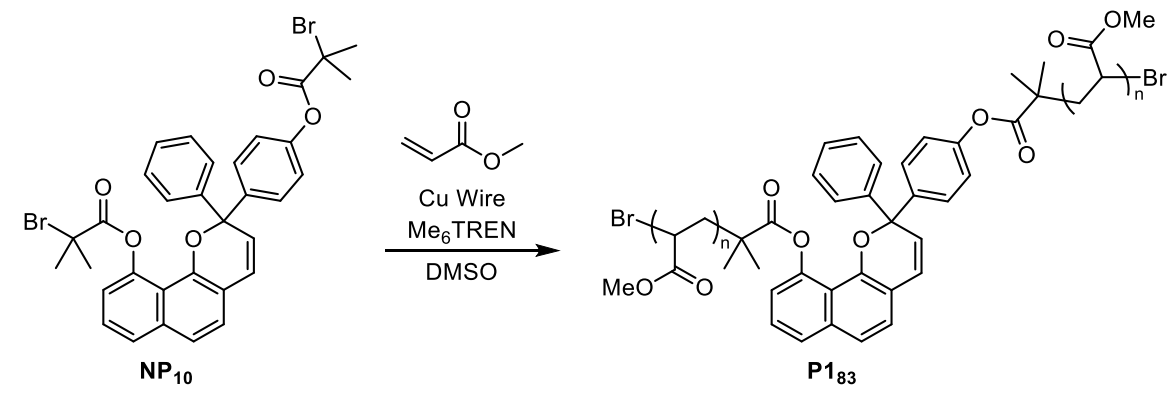

Polymer $\mathbf{P 1}_{\mathbf{8 3}}$. Synthesized using General Procedure B with initiator $\mathbf{N P}_{\mathbf{1 0}}$ (16.7 $\mathrm{mg}, 0.0251 \mathrm{mmol}$ ), methyl acrylate $(2.6 \mathrm{~mL}, 29 \mathrm{mmol})$, DMSO $(2.6 \mathrm{~mL})$, and Me $\mathrm{M}_{6} \mathrm{TREN}(34 \mu \mathrm{L}, 0.13 \mathrm{mmol})$. Polymerization for $2.5 \mathrm{~h}$ provided the title polymer as a tacky orange solid (440 mg, $25 \%) . M_{\mathrm{n}}=83.1 \mathrm{~kg} / \mathrm{mol}, \emptyset=1.15$.

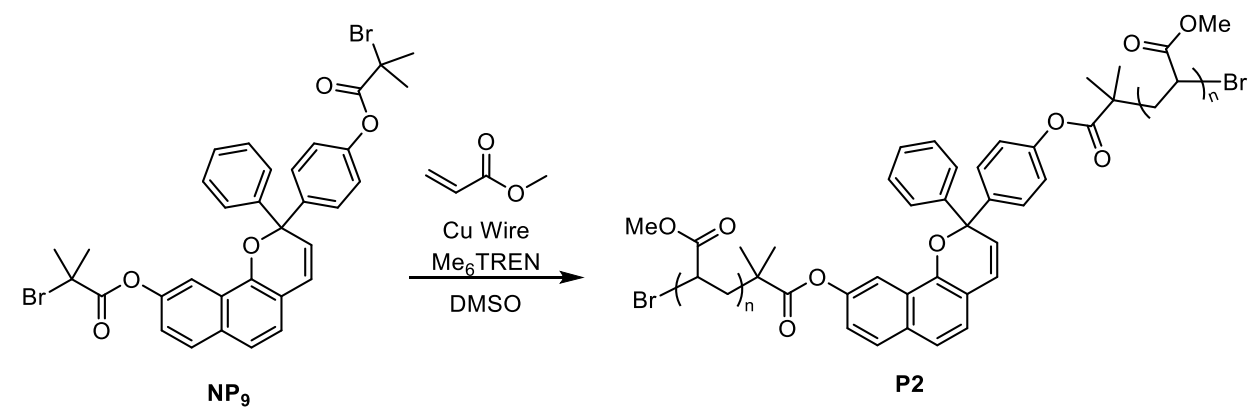

Polymer P2. Synthesized using General Procedure B with initiator NP $(13.9 \mathrm{mg}, 0.0209 \mathrm{mmol})$, methyl acrylate $(6.7 \mathrm{~mL}, 74 \mathrm{mmol}), \mathrm{DMSO}(6.7 \mathrm{~mL})$, and Me $\mathrm{T}_{6} \operatorname{TREN}(30 \mu \mathrm{L}, 0.11 \mathrm{mmol})$. Polymerization for $4.5 \mathrm{~h}$ provided the title polymer as a tacky orange solid $(2.5 \mathrm{~g}, 39 \%) . M_{\mathrm{n}}=174 \mathrm{~kg} / \mathrm{mol}, \emptyset=1.06$.

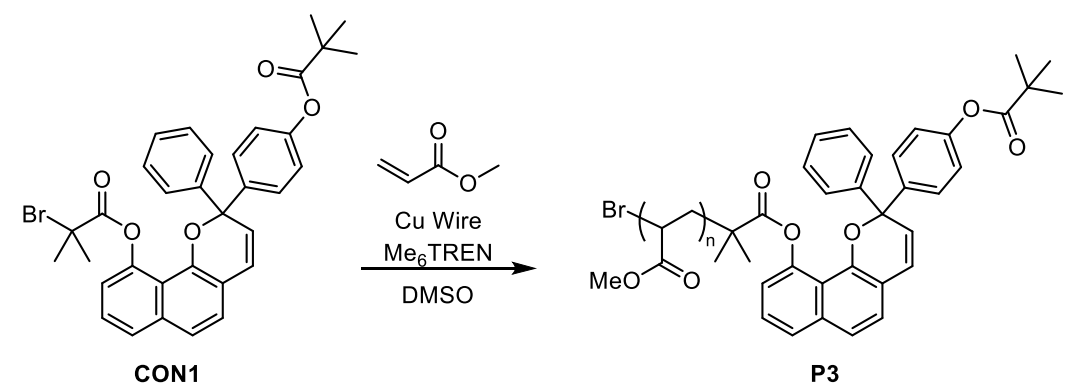

Polymer P3. Synthesized using General Procedure B with initiator CON1 (9.1 mg, $0.015 \mathrm{mmol}$ ), methyl acrylate $(5.3 \mathrm{~mL}, 59 \mathrm{mmol})$, DMSO $(5.3 \mathrm{~mL})$, and Me ${ }_{6} \operatorname{TREN}(20 \mu \mathrm{L}, 0.075 \mathrm{mmol})$. Polymerization for $3 \mathrm{~h}$ provided the title polymer as a tacky orange solid $(1.2 \mathrm{~g}, 24 \%) . M_{\mathrm{n}}=183 \mathrm{~kg} / \mathrm{mol}, \oplus=1.07$. 


\section{Characterization of Linear PMA Polymers.}
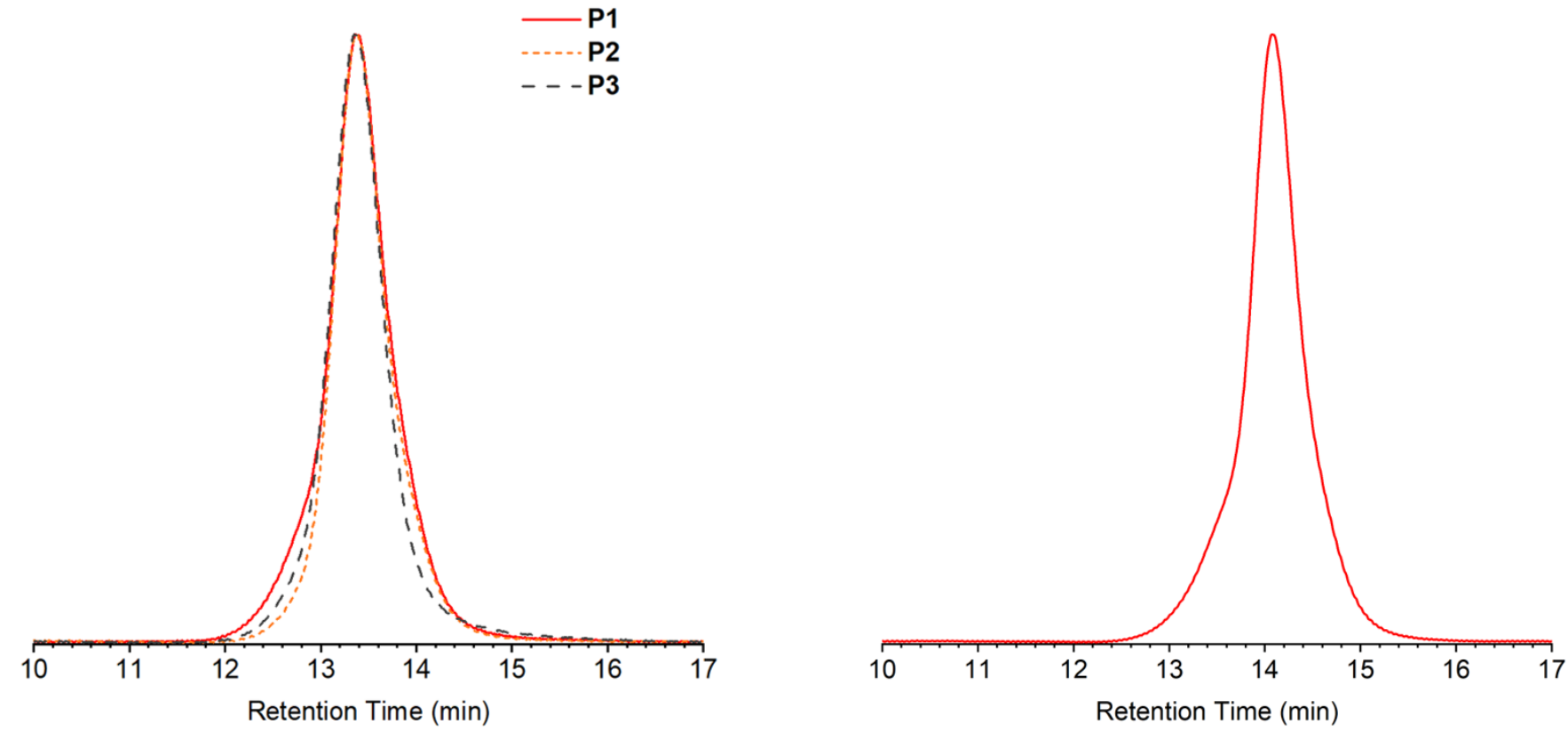

Figure 59. GPC traces (refractive index response) normalized to peak height for P1, P2, and P3 (left), and P183 (right).

(a) $\mathrm{NP}_{10},{ }^{1} \mathrm{H}\left(400 \mathrm{MHz}\right.$, acetone- $\left.d_{6}\right)$
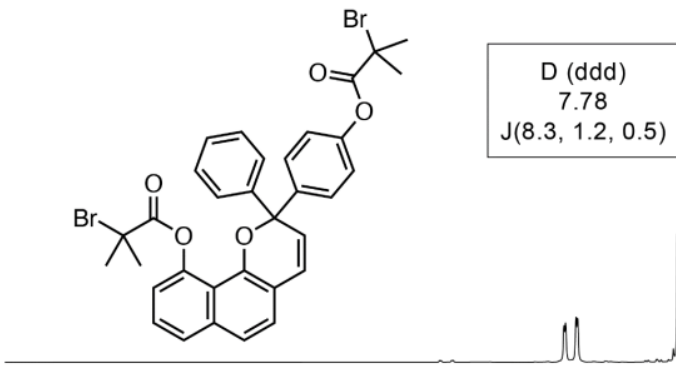

\begin{tabular}{|c|}
\hline$C(d)$ \\
7.30 \\
$J(8.4)$ \\
\hline
\end{tabular}

\begin{tabular}{|c|}
\hline$B(d)$ \\
6.84 \\
$J(9.6)$ \\
\hline
\end{tabular}

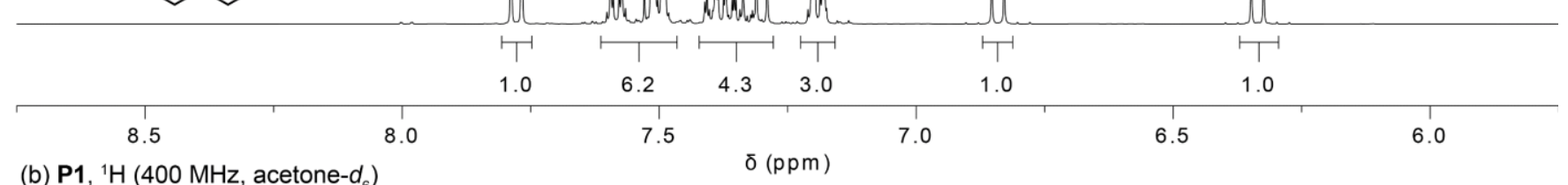

(b) $\mathrm{P} 1,{ }^{1} \mathrm{H}\left(400 \mathrm{MHz}\right.$, acetone- $\left.d_{6}\right)$

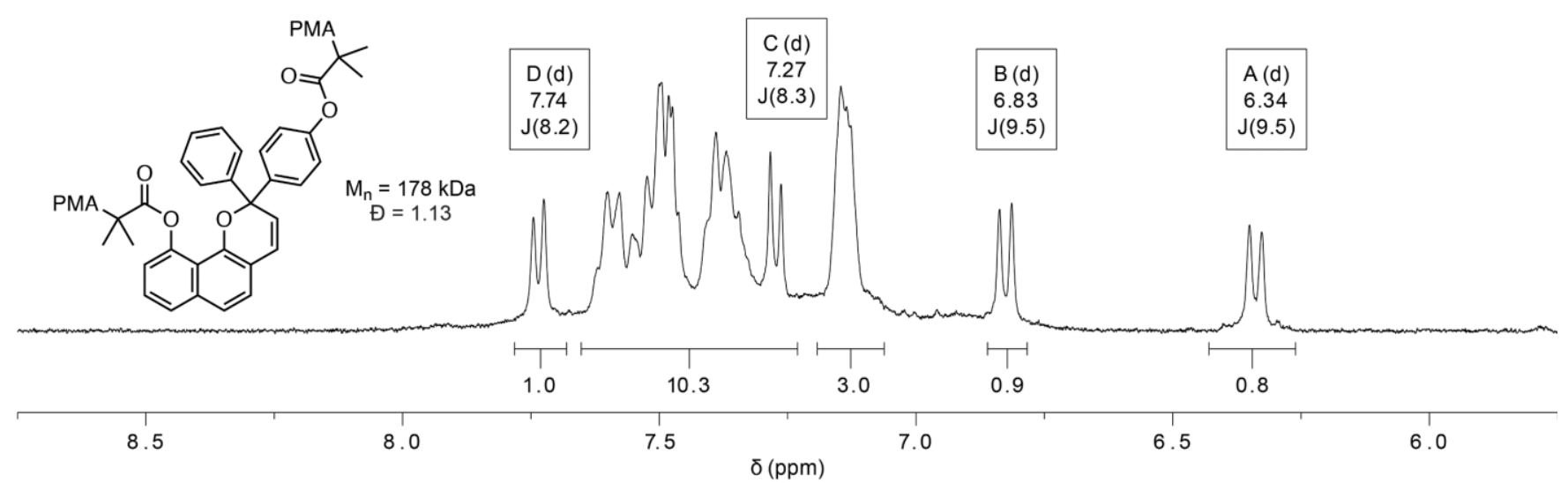

Figure S10. Partial ${ }^{1} \mathrm{H}$ NMR spectra of (a) small molecule initiator $\mathbf{N P}_{\mathbf{1 0}}$, and (b) polymer $\mathbf{P} \mathbf{1}$ demonstrating successful incorporation of the initiator into the polymer chain. 


\section{Preparation of PDMS Materials}

PDMS materials incorporating naphthopyran (1.5 wt\%) were prepared following previously reported procedures using the two-part Sylgard ${ }^{\circ} 184$ elastomer kit (Dow Corning). ${ }^{3,4}$ PDMS films approximately $0.5 \mathrm{~mm}$ thick were cut into $8 \mathrm{~mm}$ diameter disks using a hole punch unless otherwise specified.

General Procedure for Preparation of PDMS Materials. A representative procedure is provided for the

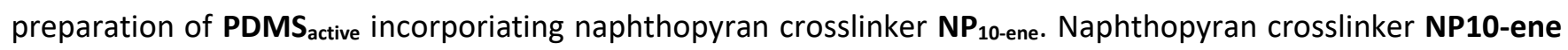
(30.4 mg) was dissolved in xylene $(0.3 \mathrm{~mL})$ in a $20 \mathrm{~mL}$ scintillation vial. Sylgard ${ }^{\circledR} 184$ prepolymer base $(1.93 \mathrm{~g})$ was added and the contents were thoroughly mixed in a vortex mixer with intermittent gentle heating to form a homogeneous, orange dispersion. Sylgard ${ }^{\circledR} 184$ curing agent $(0.193 \mathrm{~g})$ was added and the contents were mixed thoroughly using a vortex mixer. The mixture was pipetted onto a clean $5 \mathrm{~cm} \times 5 \mathrm{~cm}$ delrin plate, which was placed inside a vacuum chamber and evacuated under high vacuum ( 30 mTorr) for $3 \mathrm{~h}$. The delrin plate was then transferred to an oven and cured at $80^{\circ} \mathrm{C}$ overnight. After curing, the plate was removed from the oven and the PDMS film was peeled off and either cut into strips with a razor blade or cut into uniform $8 \mathrm{~mm}$ circles using a

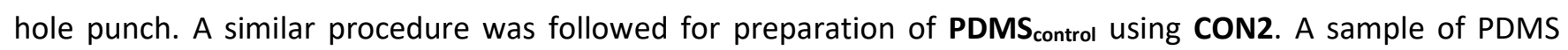
without any additional naphthopyran was also prepared similarly as a blank. PDMS samples containing naphthopyran were irradiated with blue light $(470 \mathrm{~nm})$ for $30 \mathrm{~min}$ to reduce initial coloration.

\section{DFT Calculations (CoGEF)}

CoGEF calculations were performed using Spartan '18 Parallel Suite according to previously reported methods. 5,6 Ground state energies were calculated using DFT at the B3LYP/6-31G* level of theory. For each mechanophore, the equilibrium conformations of the unconstrained molecule was initially calculated using molecular mechanics (MMFF) followed by optimization of the equilibrium geometries using DFT (B3LYP/6-31G*). Starting from the equilibrium geometry of the unconstrained molecules (energy $=0 \mathrm{~kJ} / \mathrm{mol}$ ), the distance between the terminal methyl groups of the truncated structures was increased in increments of $0.05 \AA$ and the energy was minimized at each step. The maximum force associated with the mechanochemical reaction was calculated from the slope of the curve immediately prior to bond cleavage. CoGEF results are shown in Figure S5 for a truncated model reflecting the mechanophore in $\mathbf{P 1}$, while the results for a truncated model reflecting the mechanophore in $\mathbf{P 2}$ with attachment at the 9-position of the naphthopyran are illustrated below in Figure S11. In contrast to the C-O bond scission reaction predicted for the naphthopyran in $\mathbf{P 1}$, the naphthopyran in $\mathbf{P} \mathbf{2}$ is predicted to undergo cleavage of the terminal $\mathrm{C}-\mathrm{C}$ bond upon full extension. 


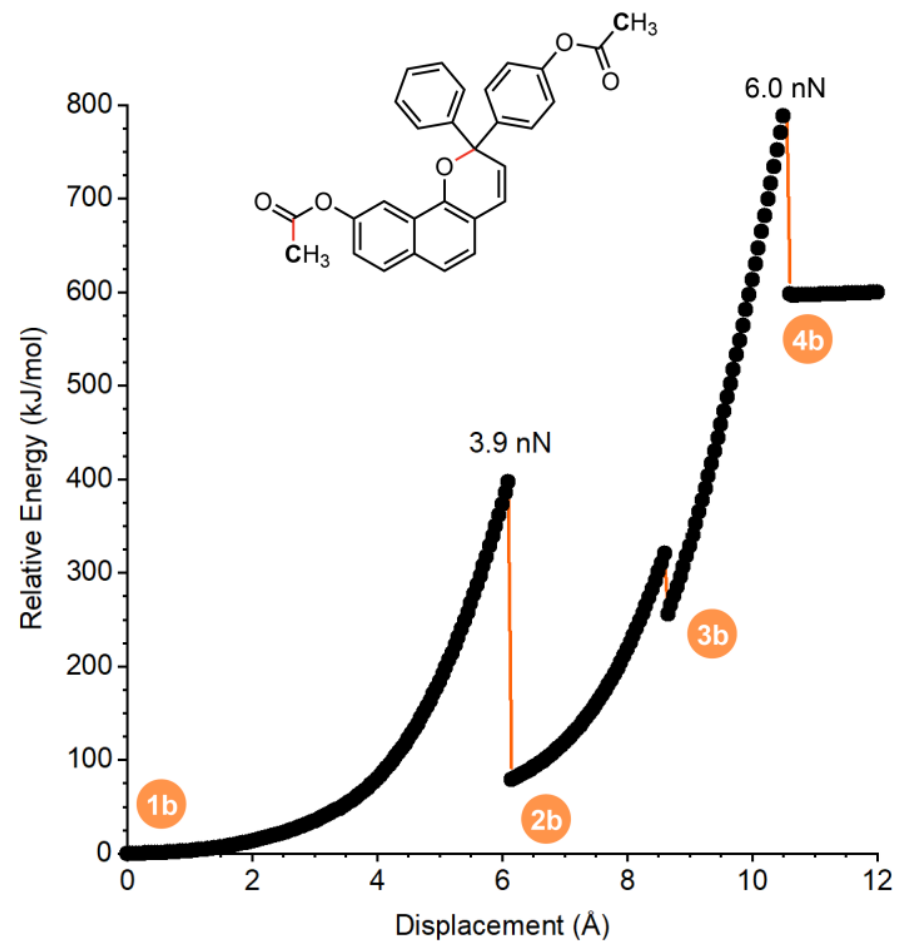

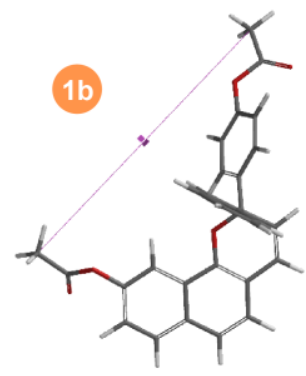

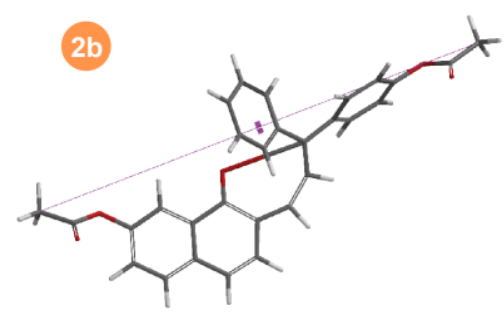

$12.483 \AA$
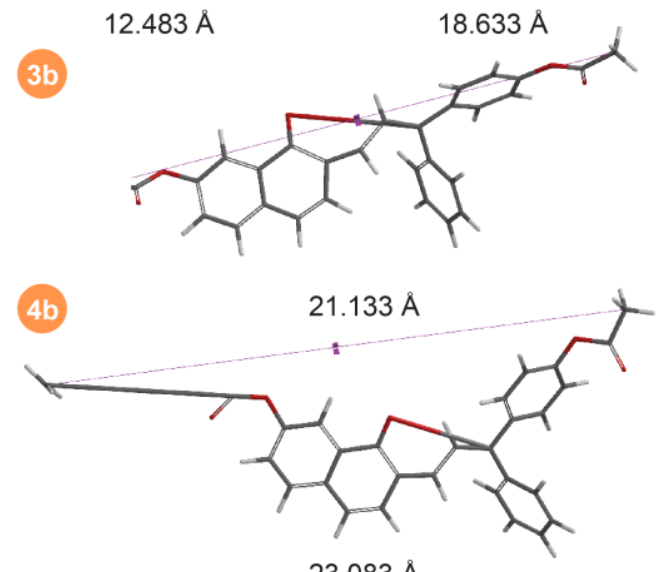

$23.083 \AA$

Figure S11. DFT calculations using the constrained geometries simulate external force (CoGEF) method at the B3LYP/6-31G* level of theory for a truncated model reflecting the mechanophore in polymer P2. CoGEF calculations predict the ring-opening reaction with an $F_{\max }$ value of $3.9 \mathrm{nN}$ followed by further elongation that results in $\mathrm{C}-\mathrm{C}$ bond scission at a predicted $F_{\text {max }}$ value of $6.0 \mathrm{nN}$. The corresponding computed structures at various points of elongation are shown at right along with the associated constraint distance between the terminal methyl groups.

\section{Details for Photoirradiation and Sonication Experiments}

In order to continuously monitor reaction progress by UV-vis absorption spectroscopy, a previously reported experimental setup ${ }^{7,8}$ was assembled using a peristaltic pump to transport solution from the reaction vessel through a quartz flow cell in a UV-vis spectrometer and return the solution to the reaction vessel. The flow rate through the system was maintained at $8 \mathrm{~mL} / \mathrm{min}$, corresponding to a setting of 50 RPM on the peristaltic pump at the selected occlusion. The UV-vis spectrometer was programmed to acquire either full spectra or absorbance at predefined wavelengths at regular time intervals. Absorbance measurements at wavelengths of $470,485,510 \mathrm{~nm}$, and $700 \mathrm{~nm}$ were acquired every $10 \mathrm{~s}$ during continuous photoirradiation or sonication of polymer solutions. The absorbance values measured at $700 \mathrm{~nm}$ were subtracted from the absorbance values monitored at 470,485 , or $510 \mathrm{~nm}$ at each time point to account for drift during the experiments. The two traces in Figure $1 \mathrm{~b}$ are normalized to their respective maximum absorbance values.

General Procedure for Sonication Experiments. A sonication vessel was placed onto the sonication probe and allowed to cool under a stream of $\mathrm{N}_{2}$. The vessel was charged with THF, which contained $30 \mathrm{mM} \mathrm{BHT} \mathrm{(19.0} \mathrm{mL)} \mathrm{to}$ avoid decomposition side reactions resulting from free radicals generated during sonication. ${ }^{9}$ An additional 6.2 $\mathrm{mL}$ of stabilized THF was pumped into the dead space of the circulatory setup. Teflon inlet and outlet tubes were inserted into the solution in the sonication vessel through punctured septa, and the pump was engaged to start the flow of solution through the system. The sonication vessel was submerged in an ice bath and the solution was sparged with $\mathrm{N}_{2}$ for 30 min. The system was then maintained under an inert atmosphere for the duration of 
sonication. Continuous sonication at $20 \mathrm{kHz}\left(8.77 \pm 0.19 \mathrm{~W} / \mathrm{cm}^{2}\right)$ was initiated and run for approximately $5 \mathrm{~min}$ to allow the temperature inside the reaction vessel to equilibrate to $15-20{ }^{\circ} \mathrm{C}$, as measured by a thermocouple inserted into the solution (Digi-Sense EW-91428-02 thermometer with Digi-Sense probe EW-08466-83). Separately, a concentrated solution of polymer $\left(1.0 \mathrm{~mL}, 52.4 \mathrm{mg} / \mathrm{mL}\right.$ in stabilized THF) was sparged with $\mathrm{N}_{2}$ for 30 min. This solution was then injected into the sonication vessel to provide a total system volume of $26.2 \mathrm{~mL}(2.0$ $\mathrm{mg} / \mathrm{mL}$ of polymer) and reaction progress was monitored by UV-vis absorption spectroscopy. Sonication intensity was calibrated via the literature method. ${ }^{10}$ The entire system was kept in the dark for the duration of the experiment.

General Procedure for Photoirradiation Experiments. To monitor thermal reversion of the photochemically generated merocyanine, a two-sided quartz cuvette was charged with a solution of the polymer in THF (2.0 $\mathrm{mg} / \mathrm{mL}, 1.0 \mathrm{~mL}$, with $1 \mathrm{mM} \mathrm{BHT})$ and then exposed to a UV light source $(\lambda=311 \mathrm{~nm}$ ) positioned 2 in away for either $10 \mathrm{~s}$ or $10 \mathrm{~min}$ (see Figure S3). The cuvette was immediately placed into the spectrometer and absorption was monitored over time. To monitor the photochemical ring-opening reaction, a four-sided quartz cuvette was fitted with a septum with holes for inlet and outlet tubes. The cuvette was charged with a solution of the polymer in THF ( $2.0 \mathrm{mg} / \mathrm{mL}, 3.0 \mathrm{~mL}$, with $1 \mathrm{mM} \mathrm{BHT}$ ). An additional $6.2 \mathrm{~mL}$ of polymer solution was pumped into the dead space of the circulatory setup. Teflon inlet and outlet tubes were inserted into the solution in the cuvette through the septum and the pump was engaged to start the flow of solution through the system. Photoirradiation experiments were performed at room temperature $\left(19-22^{\circ} \mathrm{C}\right)$. The cuvette was then exposed to a UV light source $(\lambda=311 \mathrm{~nm})$ positioned 2 in away. The total volume of the apparatus was $9.2 \mathrm{~mL}$, with $3.0 \mathrm{~mL}$ contained in the cuvette. At any given time, only $3.0 \mathrm{~mL}$ of solution (out of the total $9.2 \mathrm{~mL}$ ) was inside of the cuvette and exposed to UV irradiation. The entire system was protected from outside light for the duration of the experiment.

\section{Description of Control Experiments}

Sonication of lower molecular weight polymer $\mathbf{P} \mathbf{1}_{\mathbf{8 3}}$. To explore whether a persistent colored species is still generated at lower forces than those experienced by $\mathbf{P 1}\left(M_{\mathrm{n}}=178 \mathrm{kDa}, \emptyset=1.13\right)$, a lower molecular weight PMA polymer with the same chain-centered mechanophore was synthesized ( $\left.\mathbf{P} \mathbf{1}_{83}, M_{n}=83.1 \mathrm{kDa}, \nexists=1.15\right)$. $\mathbf{P} \mathbf{1}_{83}$ was subjected to ultrasound-induced mechanical force for $2 \mathrm{~h}$ under identical conditions as those used for P1, and then the solution was monitored for $5 \mathrm{~h}$ after cessation of ultrasound. As shown below in Figure S12, minimal reversion is observed after sonication was stopped as evidenced by the nearly constant absorbance monitored at 470 and $510 \mathrm{~nm}$. These results indicate that at lower forces transduced by shorter polymer chains, ${ }^{11}$ generation of the permanent merocyanine species is still nearly exclusively observed. Note that only partial conversion is achieved after sonication for $2 \mathrm{~h}$ due to the slower reaction kinetics compared to that of $\mathbf{P 1}$.

Sonication of chain-end functional control polymer P3. To confirm that the ring-opening and ester $\mathrm{C}(\mathrm{O})-\mathrm{O}$ cleavage reactions observed for $\mathbf{P 1}$ containing a chain-centered $2 \mathrm{H}$-naphthopyran mechanophore were due to mechanical force, a chain-end functional control polymer (P3) was synthesized and exposed to ultrasoundinduced mechanical force under identical conditions. As shown in Figure S1, no changes in absorption were detected during sonication of chain-end functional control polymer P3, supporting that the ring-opening reaction is mechanically mediated..$^{12}$ In order to rule out that the ester $\mathrm{C}(\mathrm{O})-\mathrm{O}$ cleavage is a thermally or otherwise nonmechanically mediated process, a second control experiment was devised. Chain-end functional control polymer 
P3 was first irradiated with UV light ( $311 \mathrm{~nm}, 5 \mathrm{~min}$ ) as a $52.4 \mathrm{mg} / \mathrm{mL}$ solution, and subsequently injected into the sonication vessel and subjected to continuous ultrasonication as described above. As shown below in Figure S13, the photochemically generated merocyanine steadily reverts during sonication as evidenced by the attenuation of visible absorption. In addition, a shift in $\lambda_{\max }$ is not observed, indicating that the $\mathrm{C}(\mathrm{O})-\mathrm{O}$ ester bond is not cleaved.

(a) Spectra during sonication of $\mathbf{P} \mathbf{1}_{83}$

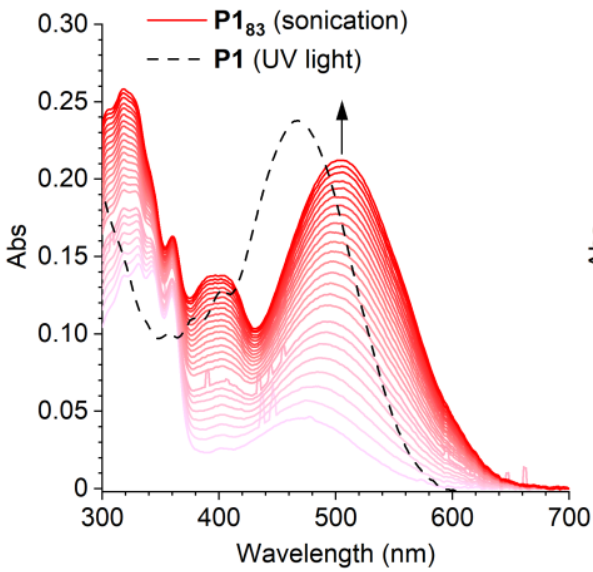

(b) Spectra after cessation of sonication of $\mathbf{P} \mathbf{1}_{83}$

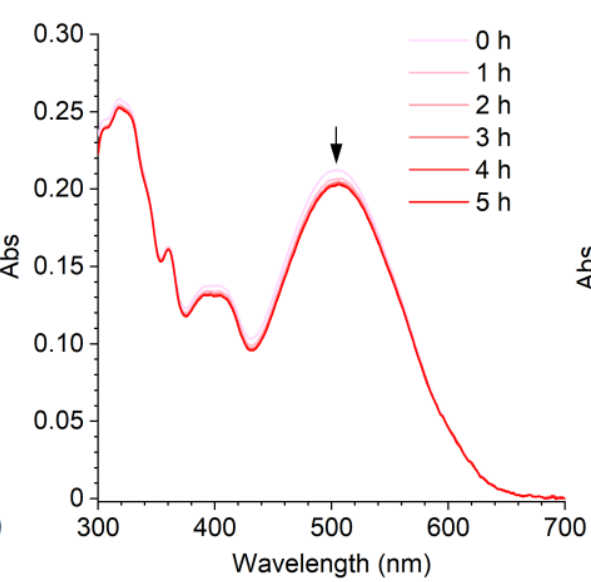

(c) Time-dependent absorbance of $\mathbf{P} \mathbf{1}_{83}$

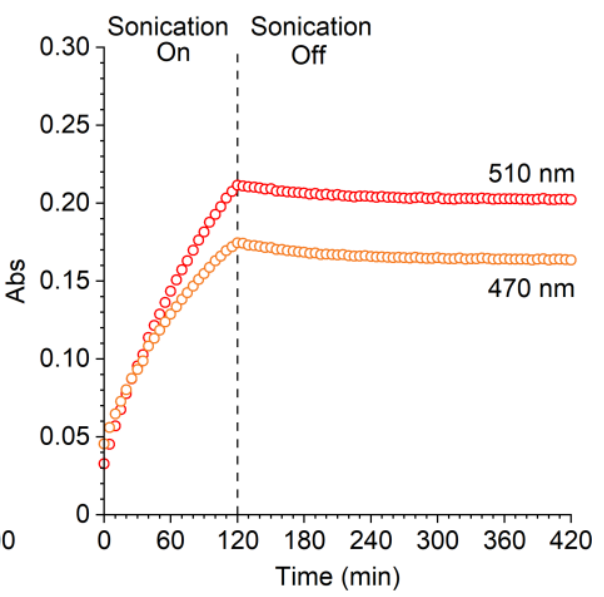

Figure S12. Characterization of ultrasound-induced mechanical activation and subsequent thermal reversion for a lower molecular weight polymer $\left(\mathbf{P} \mathbf{1}_{83}\right)$ with a chain-centered $2 \mathrm{H}-\mathrm{NP}$ mechanophore analogous to $\mathbf{P} 1$. (a) UV-vis absorption spectra acquired at regular intervals during ultrasound-induced mechanical activation of $\mathbf{P} \mathbf{1}_{\mathbf{8 3}}$ (pink and red solid traces) exhibit an increase in visible absorption that is significantly bathochromically shifted compared to the photochemical merocyanine product. Note that only partial conversion is achieved after sonication for $\mathbf{2} \mathrm{h}$. The absorption spectrum of $\mathbf{P} \mathbf{1}$ after irradiation with UV light ( $311 \mathrm{~nm}, 10 \mathrm{~min}$ ) is included for comparison with a $\lambda_{\max }$ of $470 \mathrm{~nm}$ (black dashed trace). (b,c) Spectra and timedependent absorbance at 470 and $510 \mathrm{~nm}$ acquired over a period of $5 \mathrm{~h}$ after cessation of ultrasound illustrating minimal thermal reversion.

(a) Spectral evolution during sonication of photoirradiated $\mathbf{P} 3$

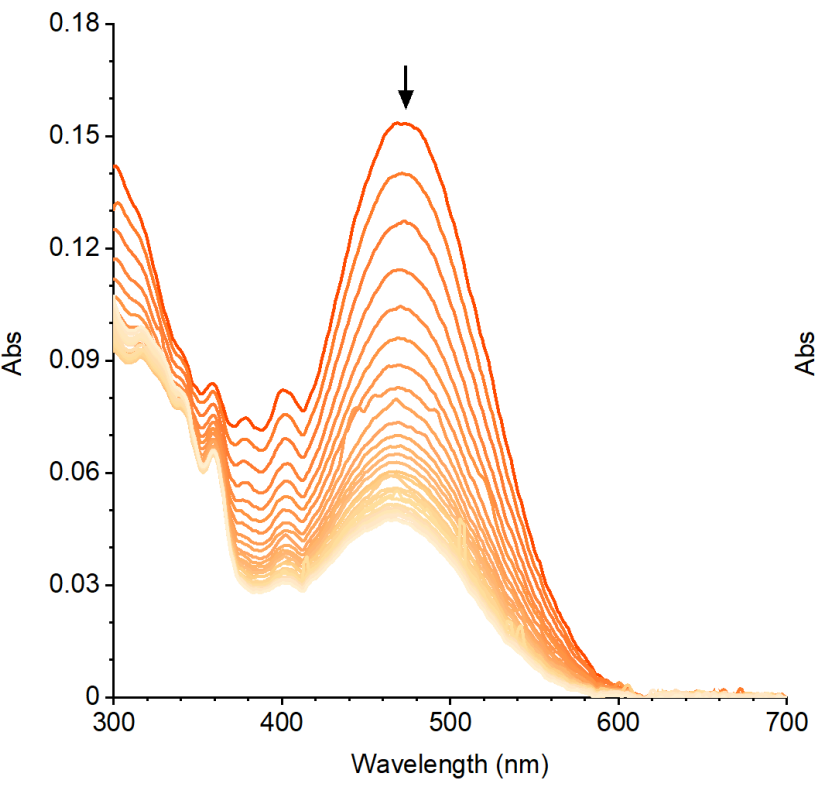

(b) Time-dependent absorbance at $470 \mathrm{~nm}$ during sonication of irrad. P3

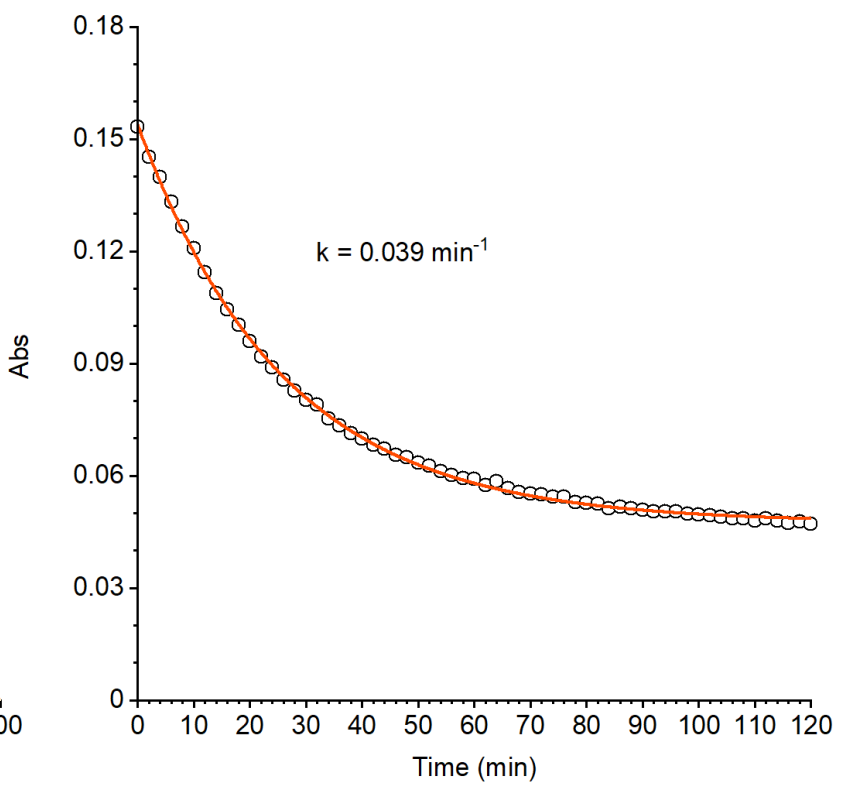

Figure S13. Sonication of chain-end control polymer P3 after initial photochemical activation with UV light does not result in a permanent merocyanine. A solution of P3 was irradiated with UV light ( $311 \mathrm{~nm}, 5 \mathrm{~min}$ ) and subsequently subjected to ultrasound. In addition, no bathochromic shift in $\lambda_{\max }$ is observed, which would occur upon ester $\mathrm{C}(\mathrm{O})-\mathrm{O}$ bond cleavage. 


\section{Kinetic Analysis}

Determination of reaction kinetics from UV-vis spectroscopy. The kinetics of mechanochemical product formation, or thermal ring-closure after UV irradiation, was evaluated by fitting time-dependent absorbance traces at $\lambda_{\max }$ to first-order exponential decay using OriginPro 2020. For tracking mechanochemical product formation, the data is fit to eq S1:

$$
A(t)=A\left(1-e^{-k t}\right)+c
$$

For tracking thermal ring-closure (UV-vis) or ultrasound-induced mechanical chain scission (GPC-RI), the timedependent signal is fit to eq S2:

$$
A(t)=A\left(e^{-k t}\right)+c
$$

Determination of polymer chain scission kinetics from GPC measurements. To determine relative rates of polymer chain scission, sonication experiments were performed on $\mathbf{P} \mathbf{1}$ and $\mathbf{P} \mathbf{2}$, during which aliquots $(1 \mathrm{~mL})$ were taken at regular time intervals for GPC analysis. Aliquots were removed from the sonication reactions via a $\mathrm{N}_{2}-$ flushed syringe during continuous sonication as described in section VI, filtered through a $0.45 \mu \mathrm{m}$ PTFE syringe filter, and submitted for GPC analysis. GPC traces for the ultrasound-induced chain scission of P1 and P2 are shown in Figure S14. GPC traces were normalized by area and the rate of chain scission was determined by plotting the attenuation of the RI response at the retention time $\left(t_{R}\right)$ corresponding to the initial polymer peak as a function of sonication time. The time dependent GPC-RI response is fit to eq S2 to determine the rate of chain scission. ${ }^{13}$

(a) Sonication of P1 monitored by GPC

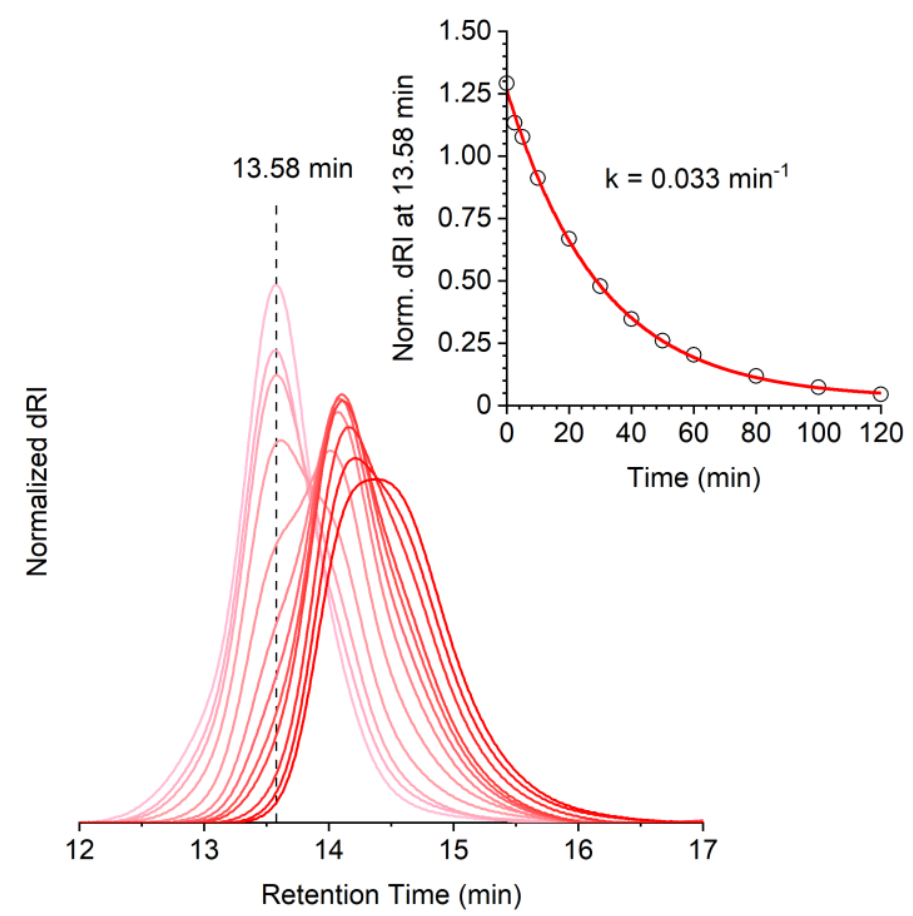

(b) Sonication of P2 monitored by GPC

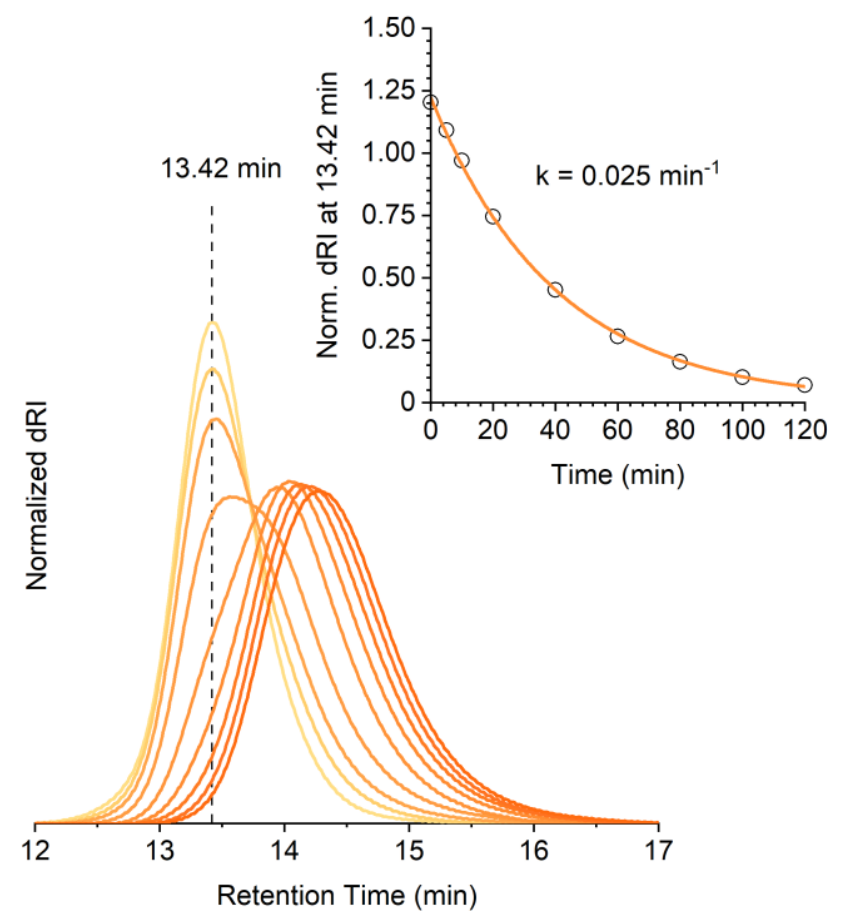

Figure S14. Ultrasound-induced mechanical chain cleavage of P1 and P2 monitored by GPC. (a) The rate of chain scission for $\mathbf{P 1}$ determined from time-dependent attenuation of the RI signal at $t_{R}=13.58 \mathrm{~min}$ was found to be $0.033 \mathrm{~min}^{-1}$. (b) The rate of chain scission for $\mathbf{P 2}$ was found to be $0.025 \mathrm{~min}^{-1}$ by monitoring the RI signal at $t_{R}=13.42 \mathrm{~min}$. 


\section{Characterization of Activation and Fading in PDMS Materials}

PDMS films were activated mechanically either manually in tension or by repeated strikes with a hammer with the film placed between sheets of weigh paper on a hard flat surface, or photochemically by irradiation with UV light $\left(\lambda_{\max }=365 \mathrm{~nm}\right)$. Films were placed between two vertical glass slides and photographed against a white background. Digital images were acquired in RAW mode with a Nikon D3200 DSLR camera. Images were cropped and standardized to a white balance temperature of 5300 and tint of +34 and an exposure of +1.50 in Adobe Photoshop using the Adobe Color profile. Reflectance measurements were performed to spectroscopically characterize the color of the PDMS materials. Spectra were acquired using an integrating sphere accessory to account for variation in surface roughness and scattering. Samples were loaded against a black sample holder. Reflectance spectra were normalized by defining the maximum and minimum value between 450 and $800 \mathrm{~nm}$ as 1 and 0 , respectively (see Figure S15). In order to make a clear comparison to solution-phase absorption data, reflectance data are plotted in Figure 4 as the inverse, i.e., " 1 - norm. reflectance".

(a)

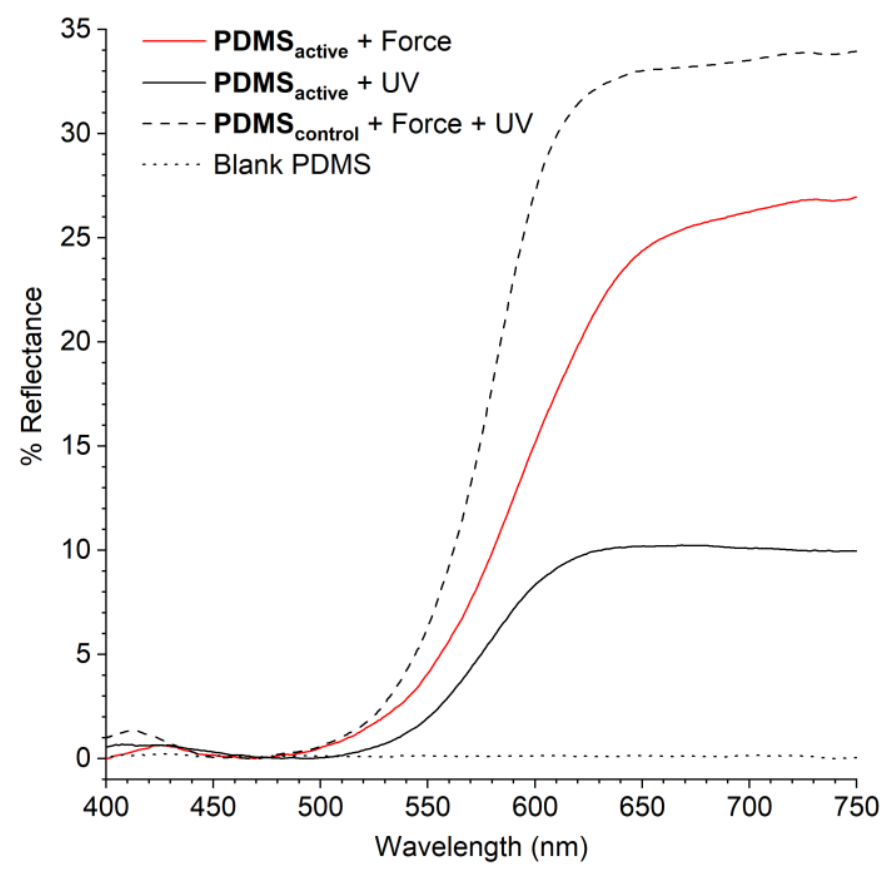

(b)

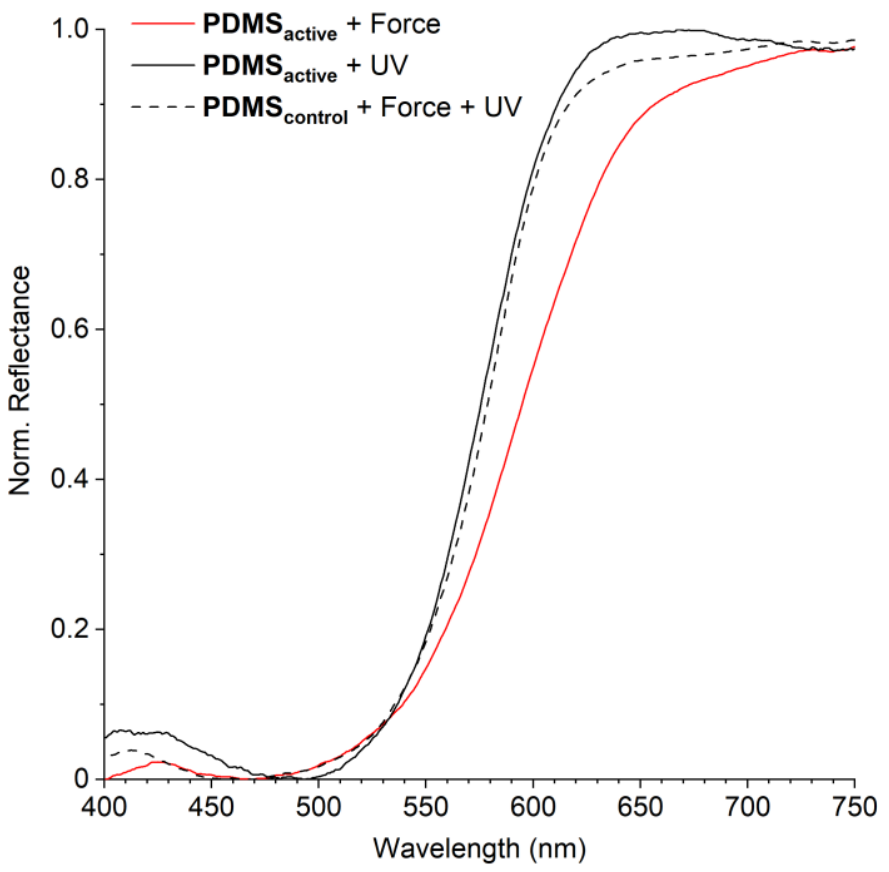

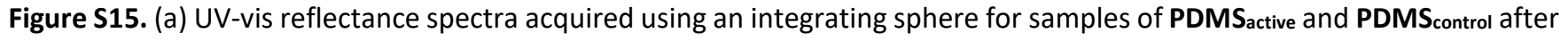
being subjected to combinations of mechanical force by repeated hammering and/or irradiation with UV light (365 nm). These are the same spectra depicted in Figure S8. The reflectance spectrum of a blank PDMS sample that does not contain naphthopyran is also shown. (b) The reflectance spectra shown in panel (a) after normalization to define the maximum value between $450-800 \mathrm{~nm}$ as 1 . 


\section{References}

(1) Zhao, W.; Carreira, E. M. Facile One-Pot Synthesis of Photochromic Pyrans. Org. Lett. 2003, 5, 4153-4154.

(2) Nguyen, N. H.; Rosen, B. M.; Lligadas, G.; Percec, V. Surface-Dependent Kinetics of Cu(0)-Wire-Catalyzed Single-Electron Transfer Living Radical Polymerization of Methyl Acrylate in DMSO at $25^{\circ} \mathrm{C}$. Macromolecules 2009, 42, 2379-2386.

(3) Gossweiler, G. R.; Hewage, G. B.; Soriano, G.; Wang, Q.; Welshofer, G. W.; Zhao, X.; Craig, S. L. Mechanochemical activation of covalent bonds in polymers with full and repeatable macroscopic shape recovery. ACS Macro Lett. 2014, 3, 216-219.

(4) Robb, M. J.; Kim, T. A.; Halmes, A. J.; White, S. R.; Sottos, N. R.; Moore, J. S. Regioisomer-Specific Mechanochromism of Naphthopyran in Polymeric Materials. J. Am. Chem. Soc. 2016, 138, 12328-12331.

(5) Beyer, M. K. The mechanical strength of a covalent bond calculated by density functional theory. J. Chem. Phys. 2000, 112, 7307-7312.

(6) Klein, I. M.; Husic, C. C.; Kovács, D. P.; Choquette, N. J.; Robb, M. J. Validation of the CoGEF Method as a Predictive Tool for Polymer Mechanochemistry. J. Am. Chem. Soc. 2020, 142, 16364-16381.

(7) May, P. A.; Munaretto, N. F.; Hamoy, M. B.; Robb, M. J.; Moore, J. S. Is Molecular Weight or Degree of Polymerization a Better Descriptor of Ultrasound-Induced Mechanochemical Transduction? ACS Macro Lett. 2016, 5, 177-180.

(8) McFadden, M. E.; Robb, M. J. Force-Dependent Multicolor Mechanochromism from a Single Mechanophore. J. Am. Chem. Soc. 2019, 141, 11388-11392.

(9) Yang, J.; Horst, M.; Werby, S. H.; Cegelski, L.; Burns, N. Z.; Xia, Y. Bicyclohexene- peri -naphthalenes: Scalable Synthesis, Diverse Functionalization, Efficient Polymerization, and Facile Mechanoactivation of Their Polymers. J. Am. Chem. Soc. 2020, 142, 14619-14626.

(10) Berkowski, K. L.; Potisek, S. L.; Hickenboth, C. R.; Moore, J. S. Ultrasound-induced site-specific cleavage of azo-functionalized poly(ethylene glycol). Macromolecules 2005, 38, 8975-8978.

(11) May, P. A.; Moore, J. S. Polymer mechanochemistry: techniques to generate molecular force via elongational flows. Chem. Soc. Rev. 2013, 42, 7497-7506.

(12) Li, J.; Nagamani, C.; Moore, J. S. Polymer Mechanochemistry: From Destructive to Productive. Acc. Chem. Res. 2015, 48, 2181-2190.

(13) Church, D. C.; Peterson, G. I.; Boydston, A. J. Comparison of Mechanochemical Chain Scission Rates for Linear versus Three-Arm Star Polymers in Strong Acoustic Fields. ACS Macro Lett. 2014, 3, 648-651. 

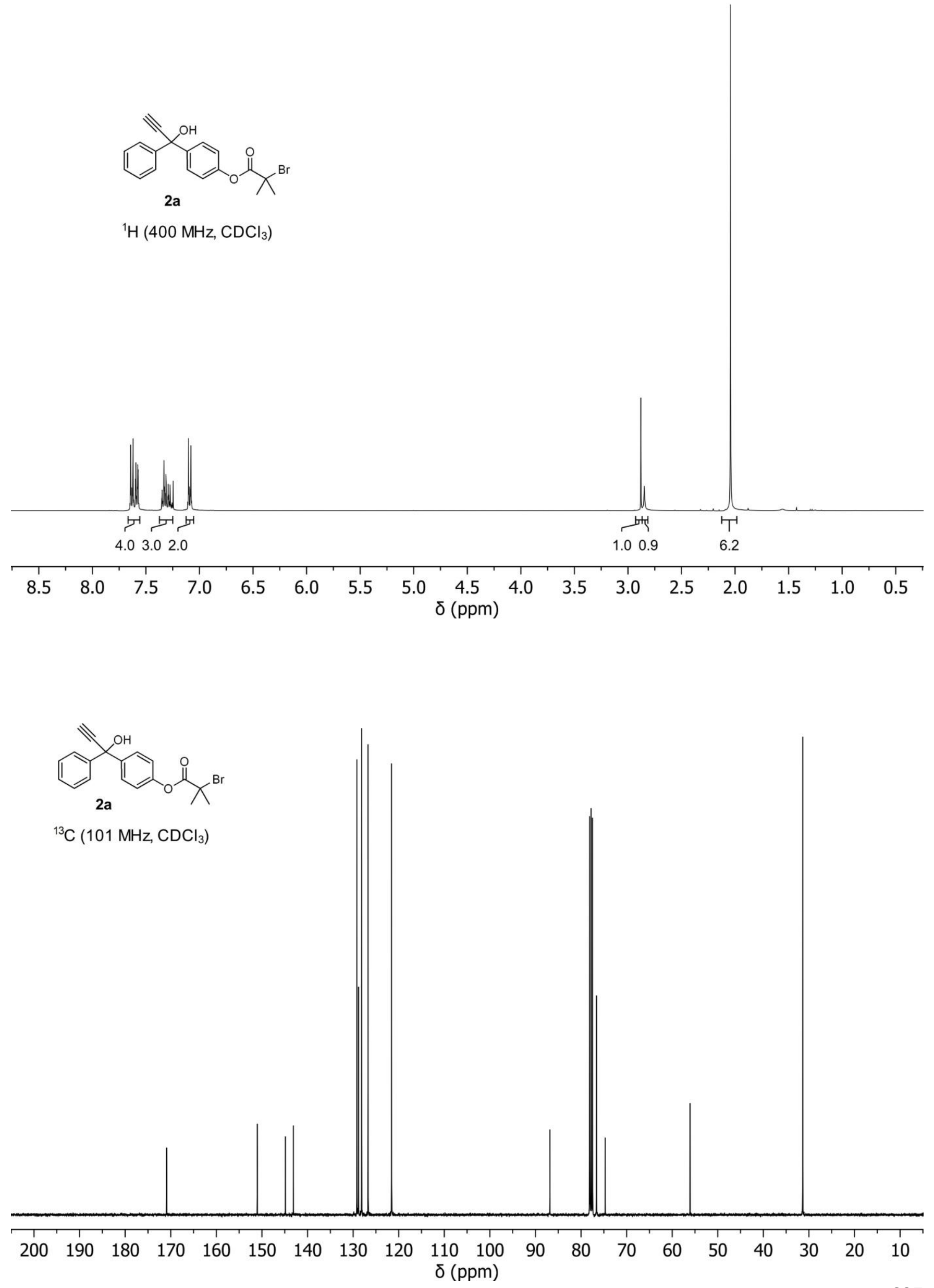


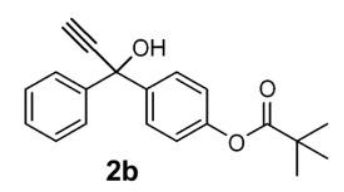

${ }^{1} \mathrm{H}\left(400 \mathrm{MHz}, \mathrm{CDCl}_{3}\right)$
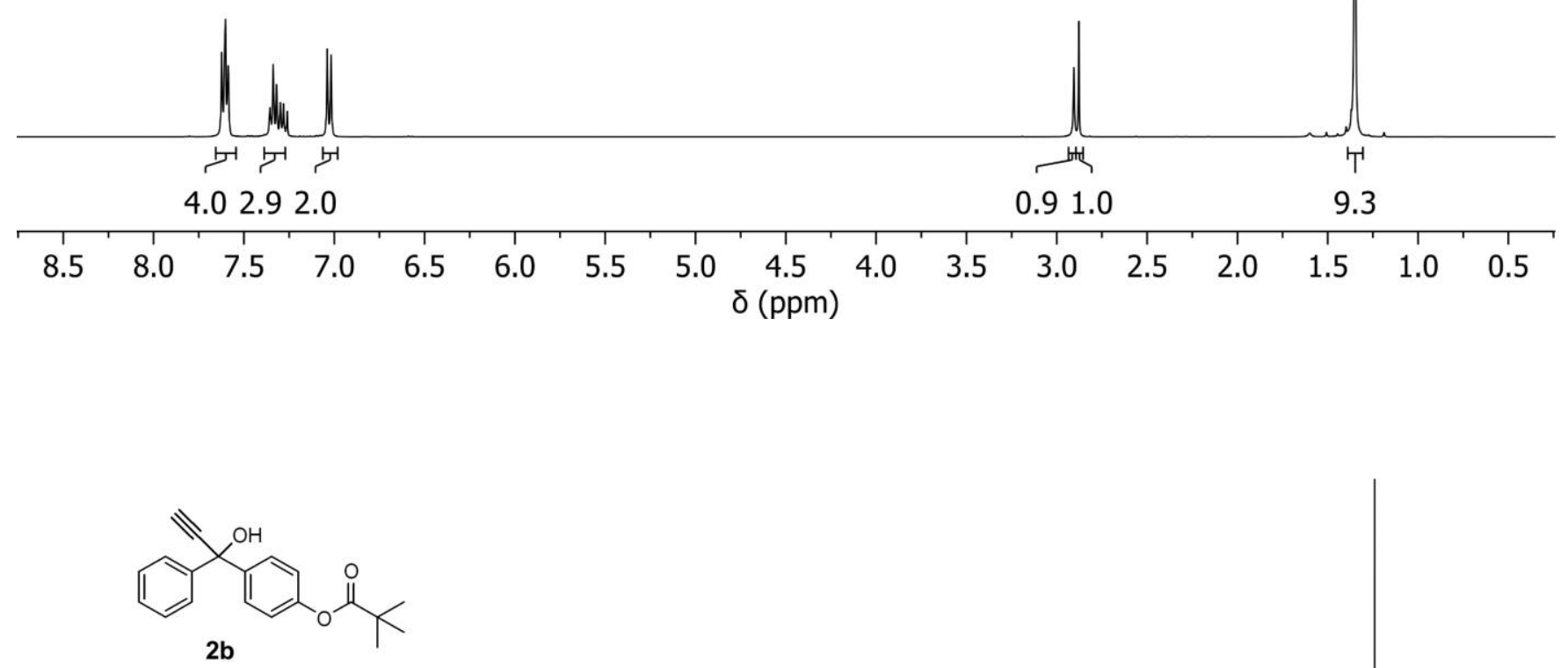

${ }^{13} \mathrm{C}\left(101 \mathrm{MHz}, \mathrm{CDCl}_{3}\right)$

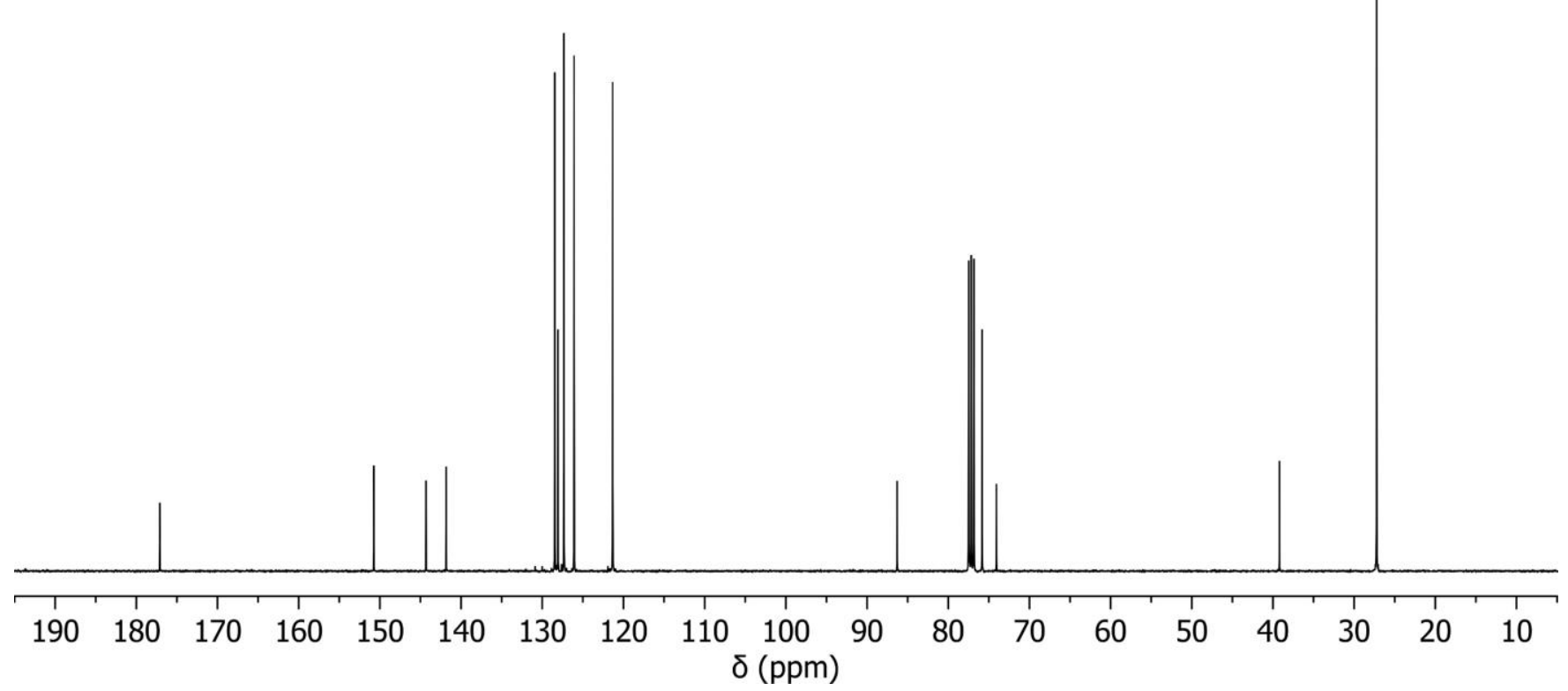



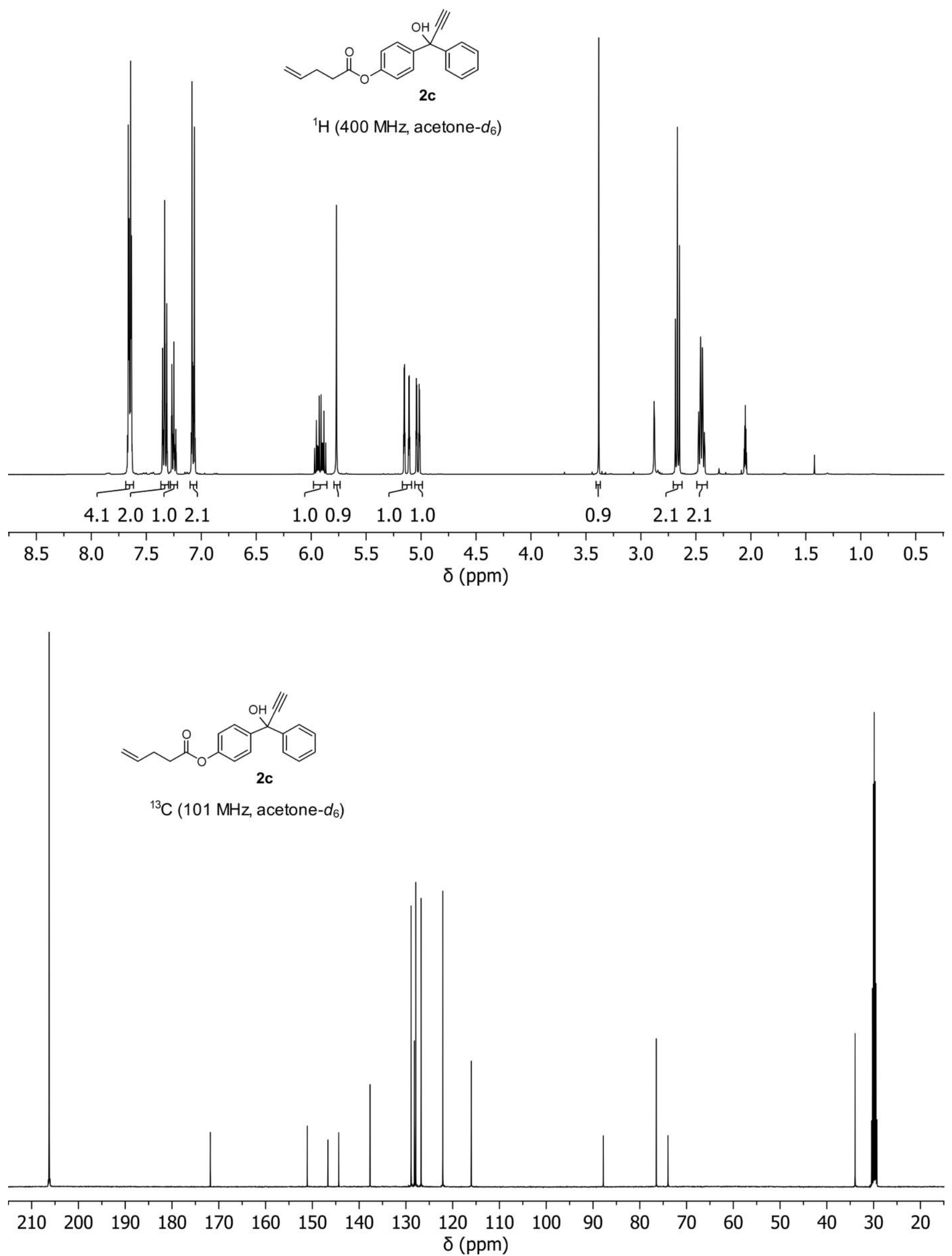


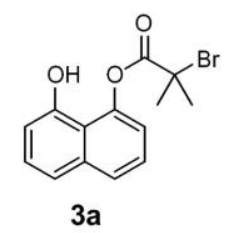

${ }^{1} \mathrm{H}\left(400 \mathrm{MHz}, \mathrm{CDCl}_{3}\right)$
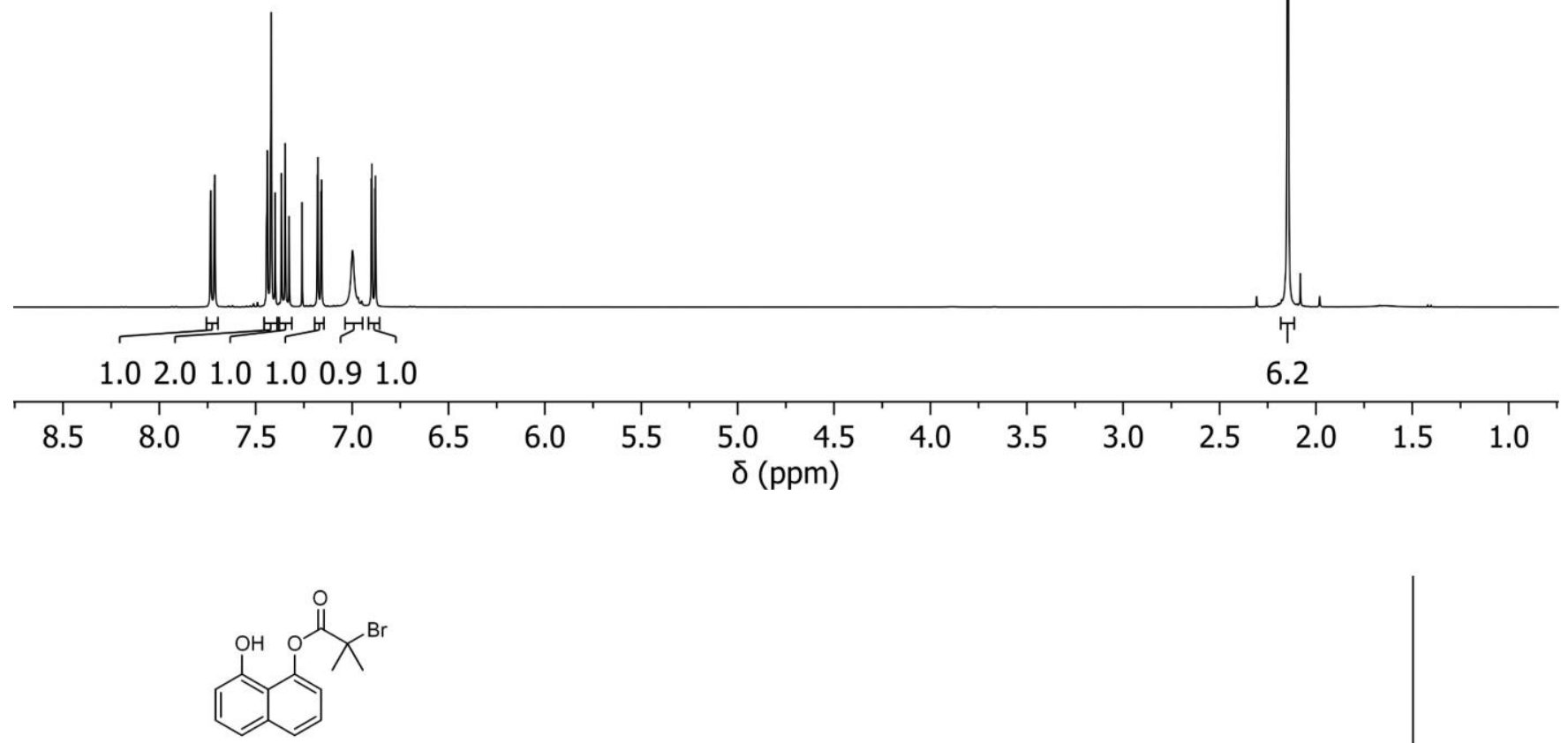

3a

${ }^{13} \mathrm{C}\left(101 \mathrm{MHz}, \mathrm{CDCl}_{3}\right)$

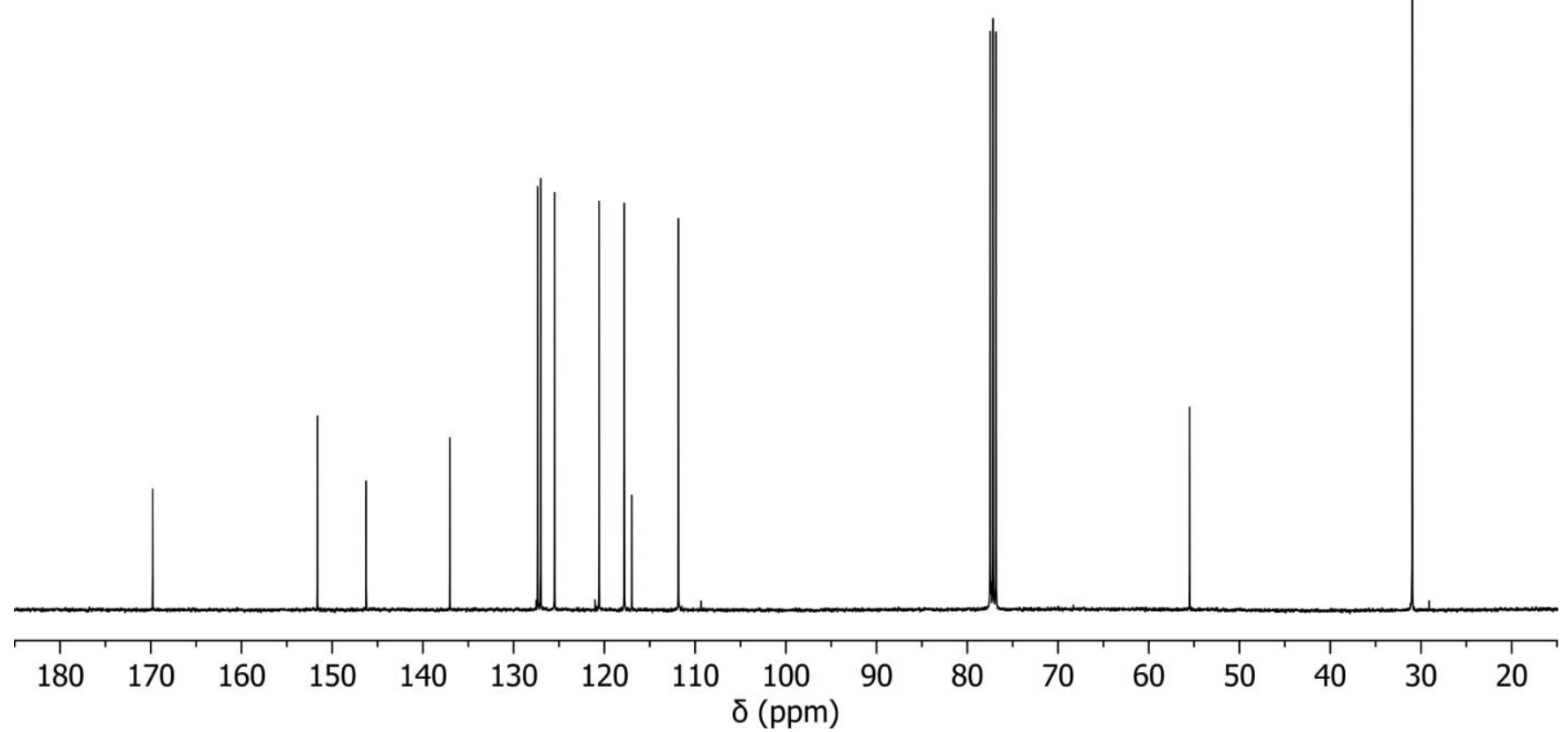




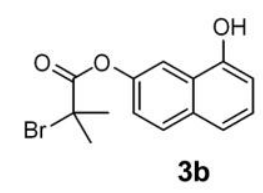

${ }^{1} \mathrm{H}\left(400 \mathrm{MHz}\right.$, acetone- $\left.d_{6}\right)$
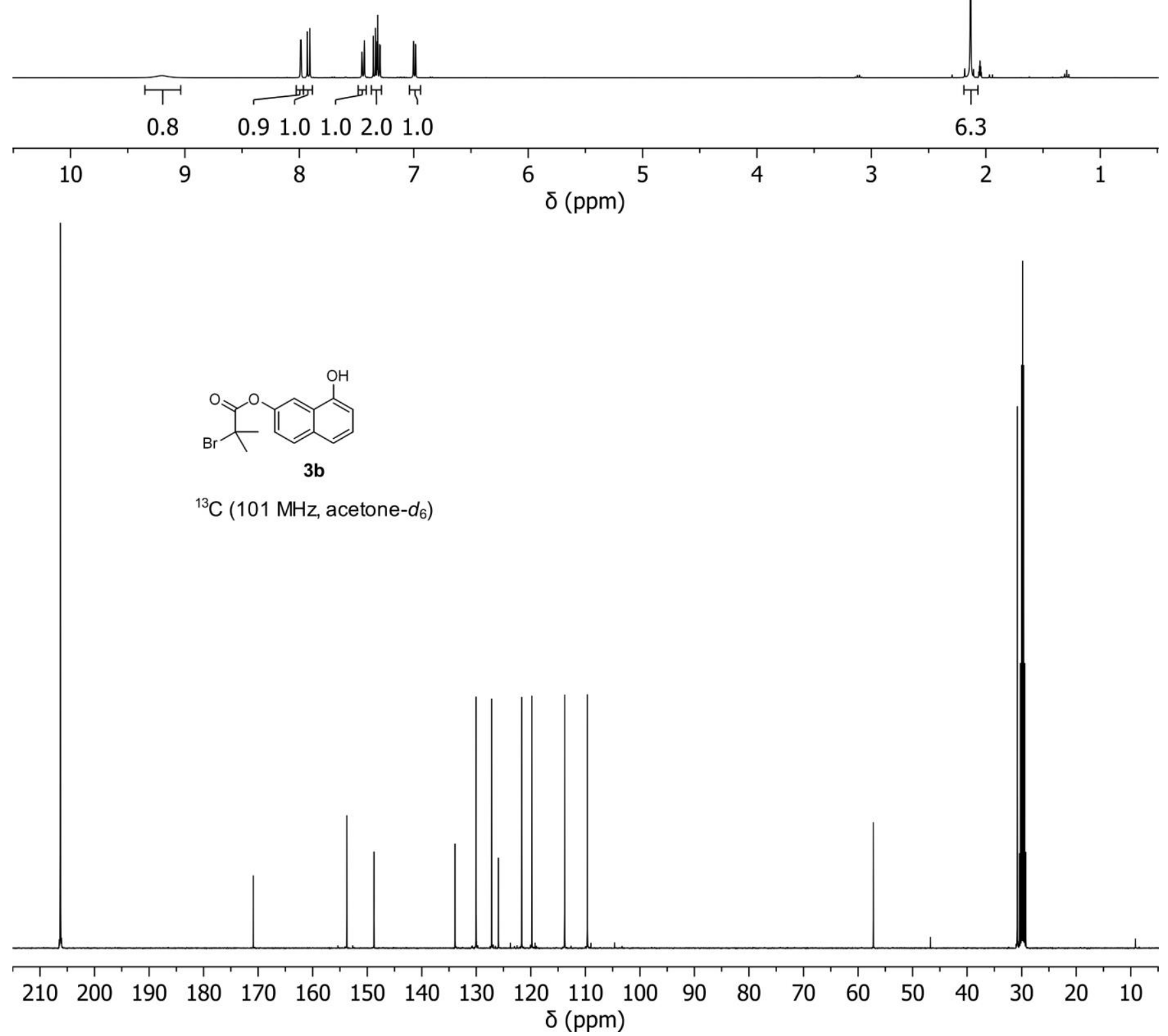


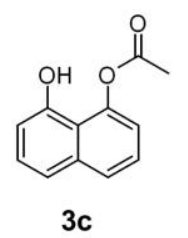

${ }^{1} \mathrm{H}\left(400 \mathrm{MHz}, \mathrm{CDCl}_{3}\right)$
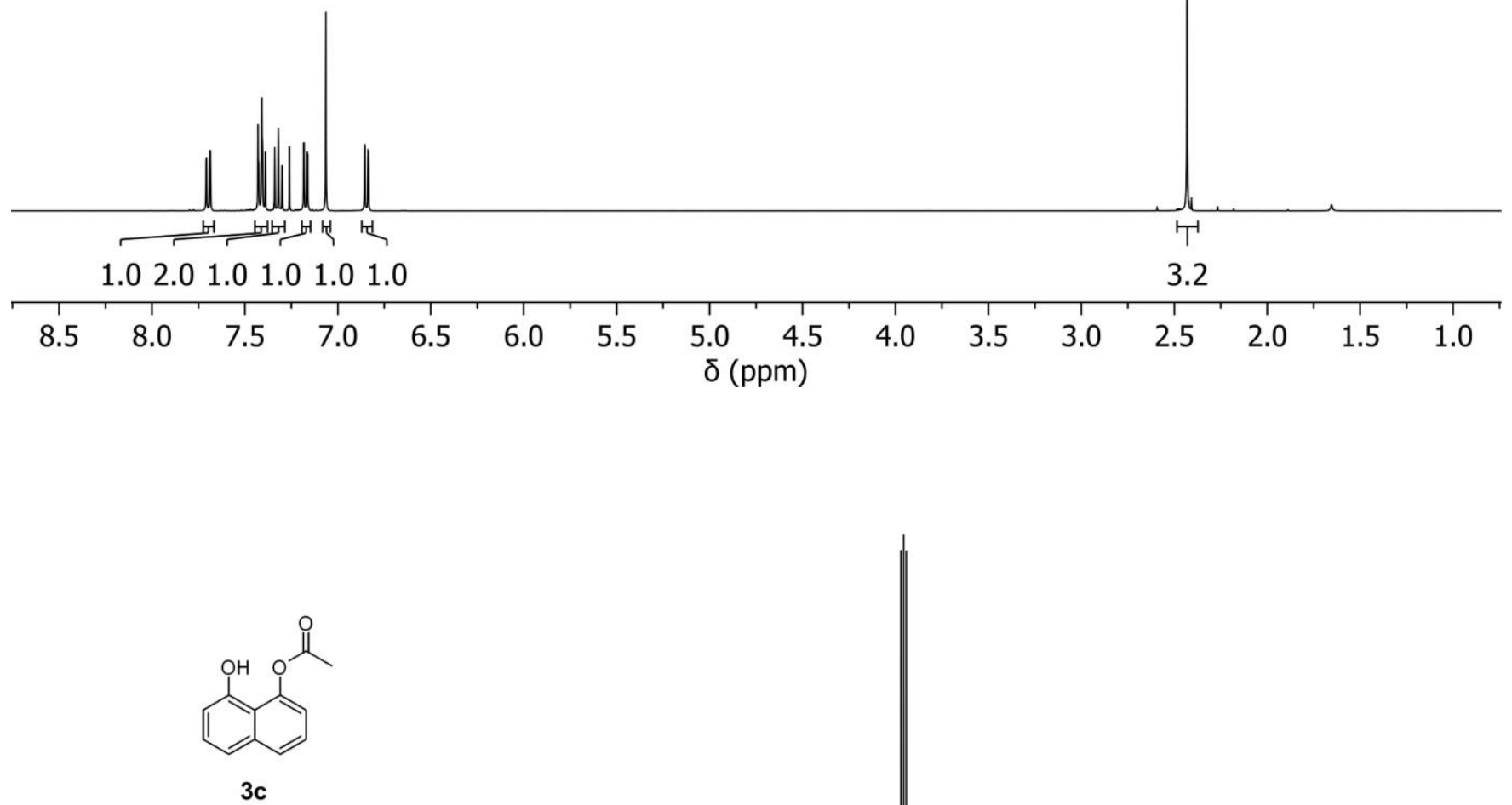

${ }^{13} \mathrm{C}\left(101 \mathrm{MHz}, \mathrm{CDCl}_{3}\right)$

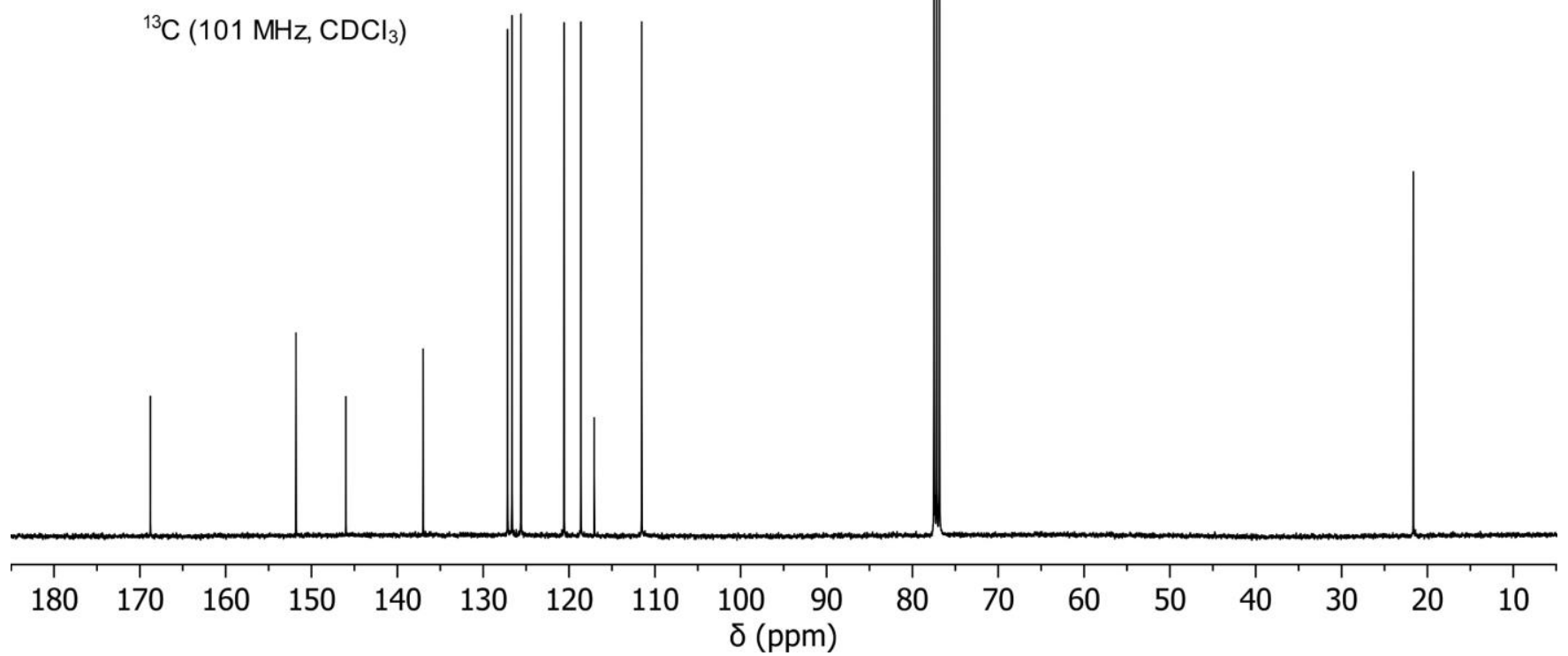



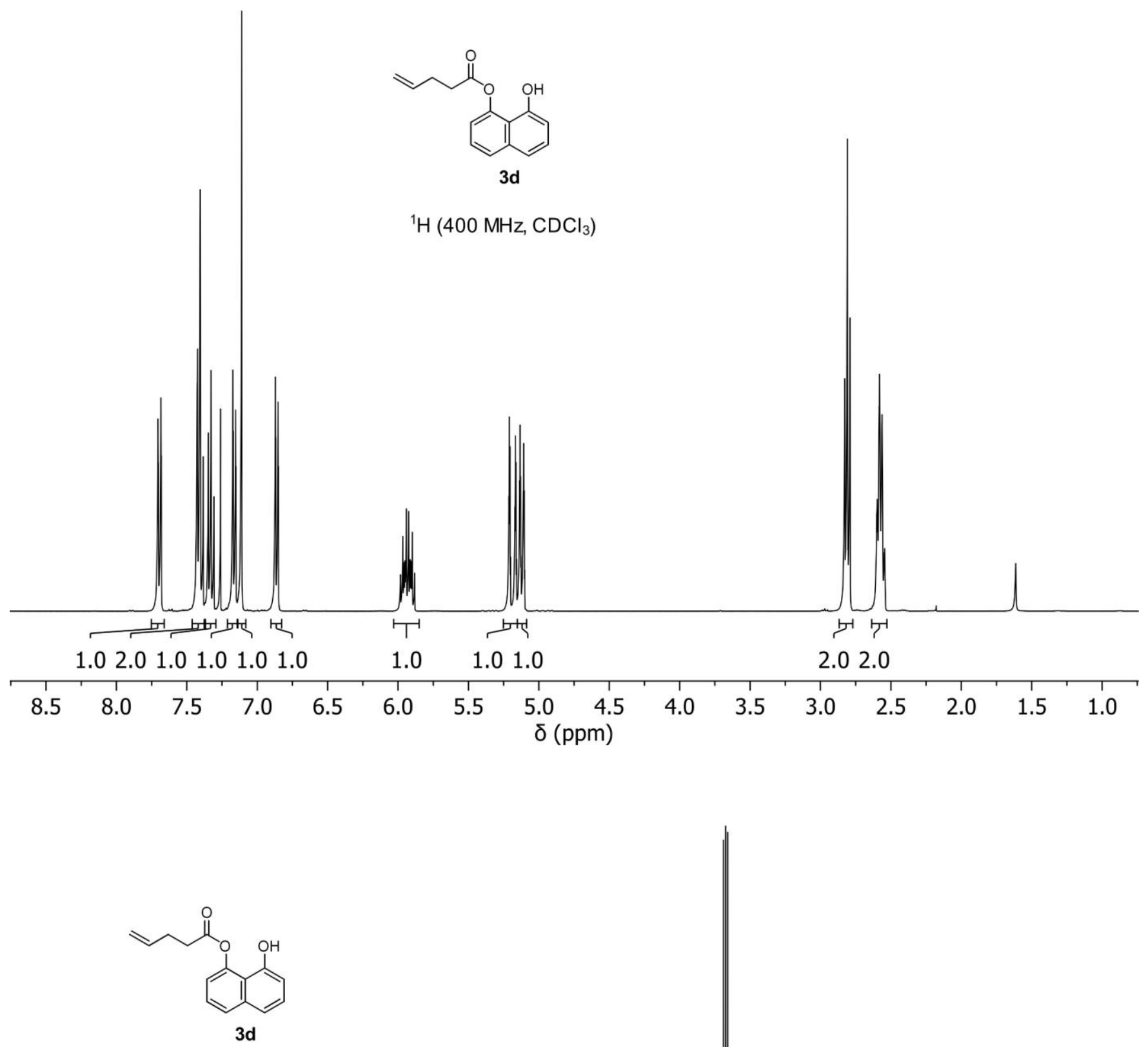

${ }^{13} \mathrm{C}\left(101 \mathrm{MHz}, \mathrm{CDCl}_{3}\right)$

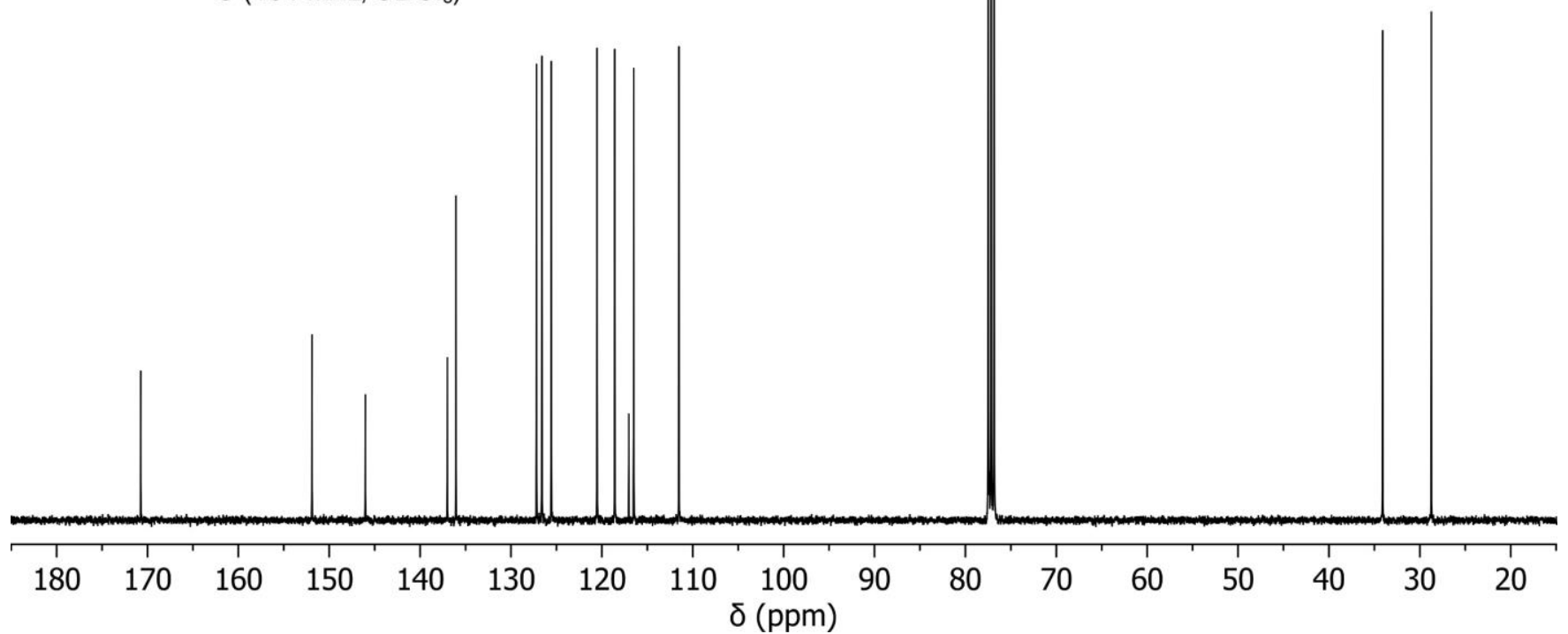




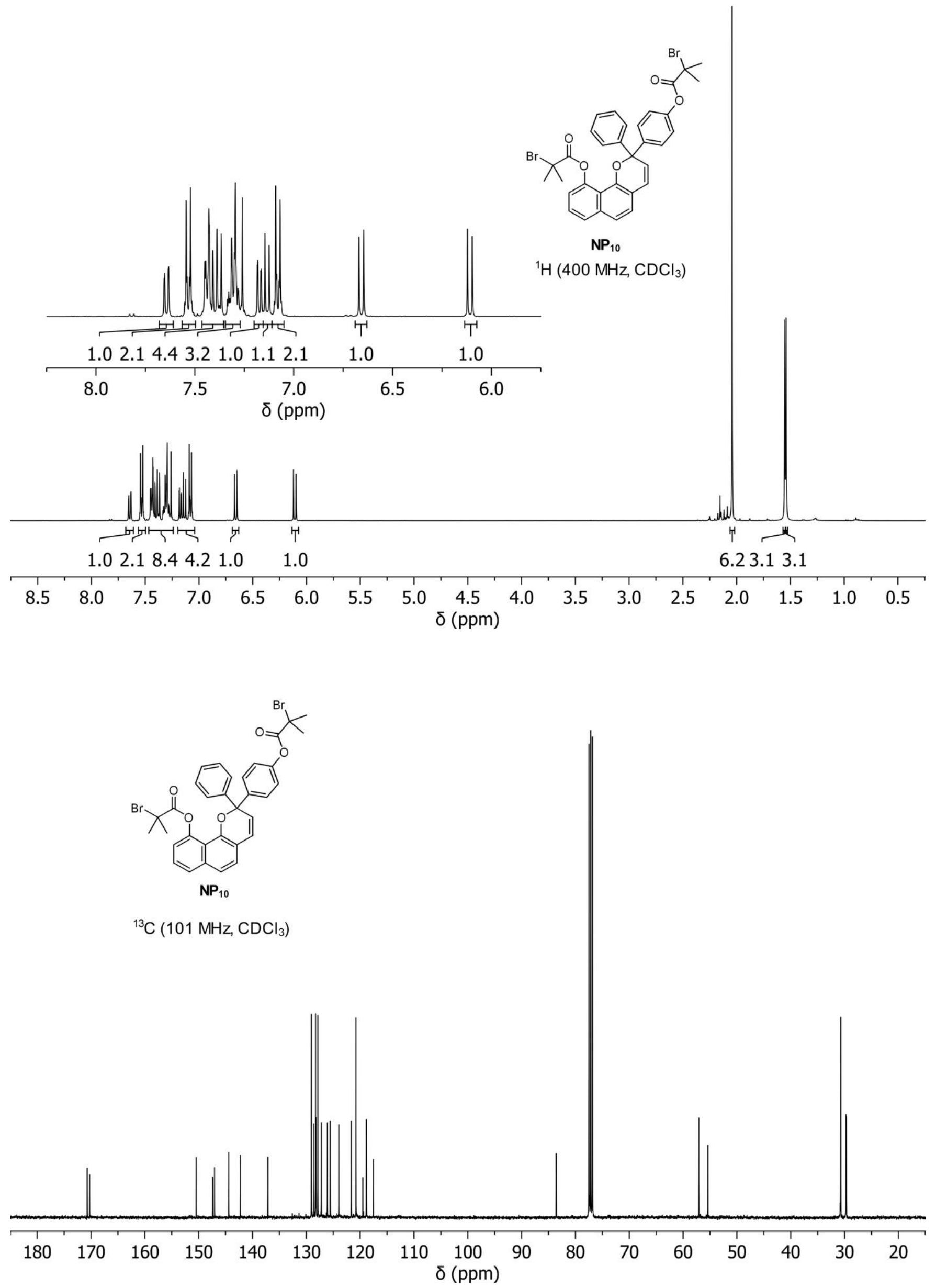




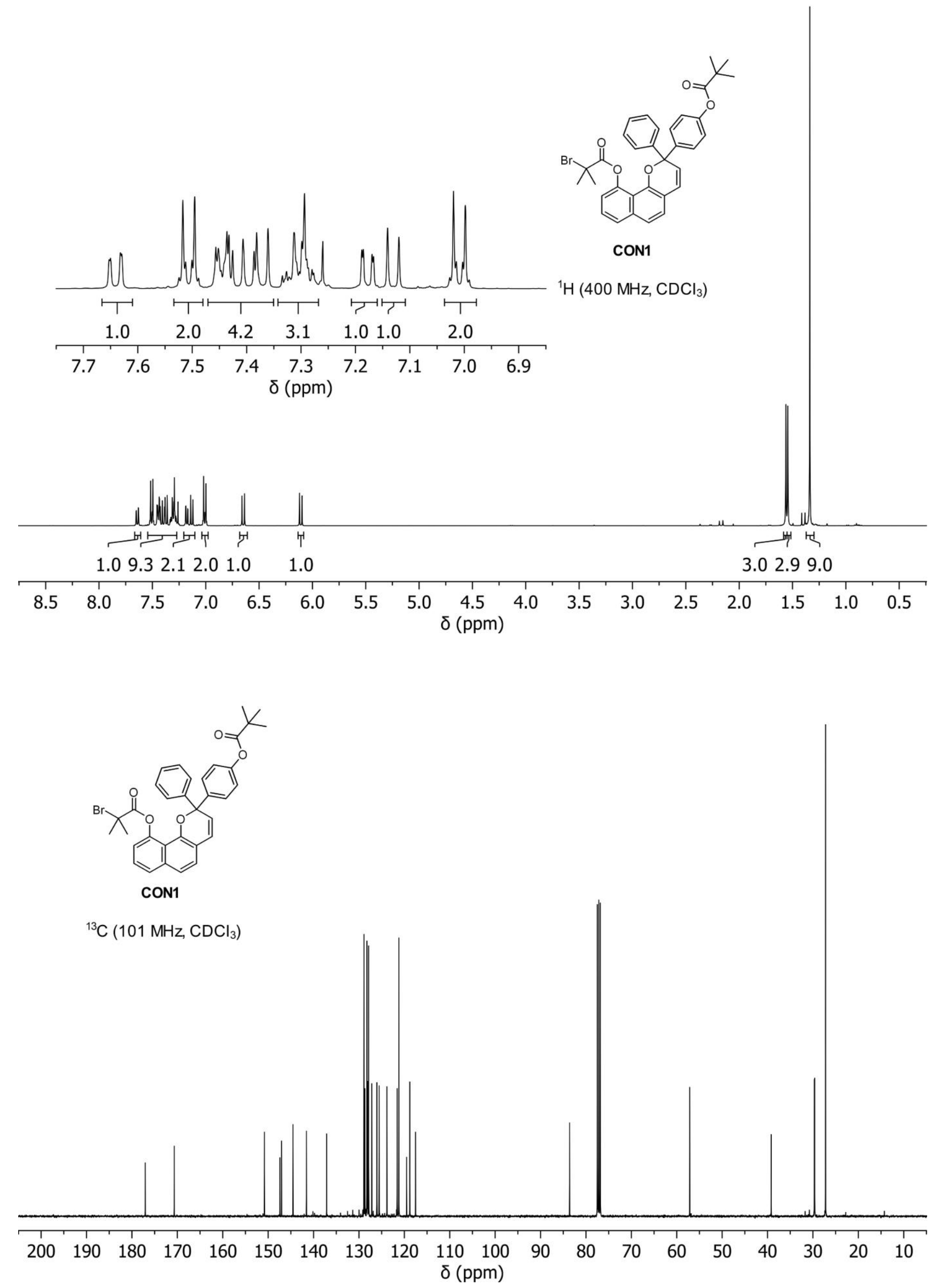



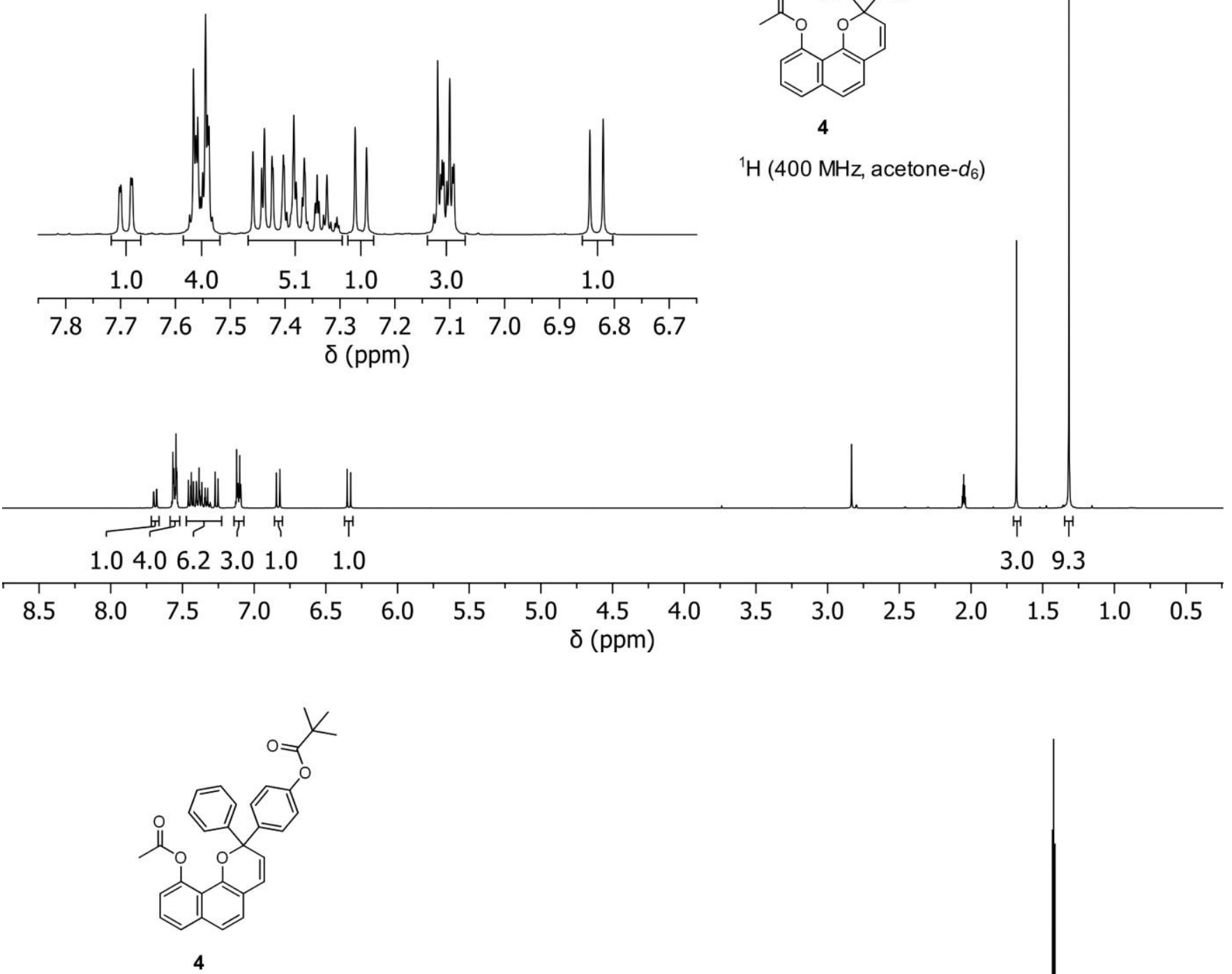

${ }^{13} \mathrm{C}\left(101 \mathrm{MHz}\right.$, acetone- $\left.d_{6}\right)$

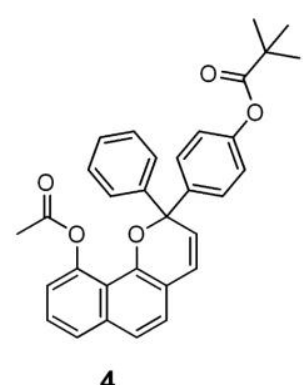

${ }^{1} \mathrm{H}$ (400 MHz, acetone- $d_{6}$ ) 

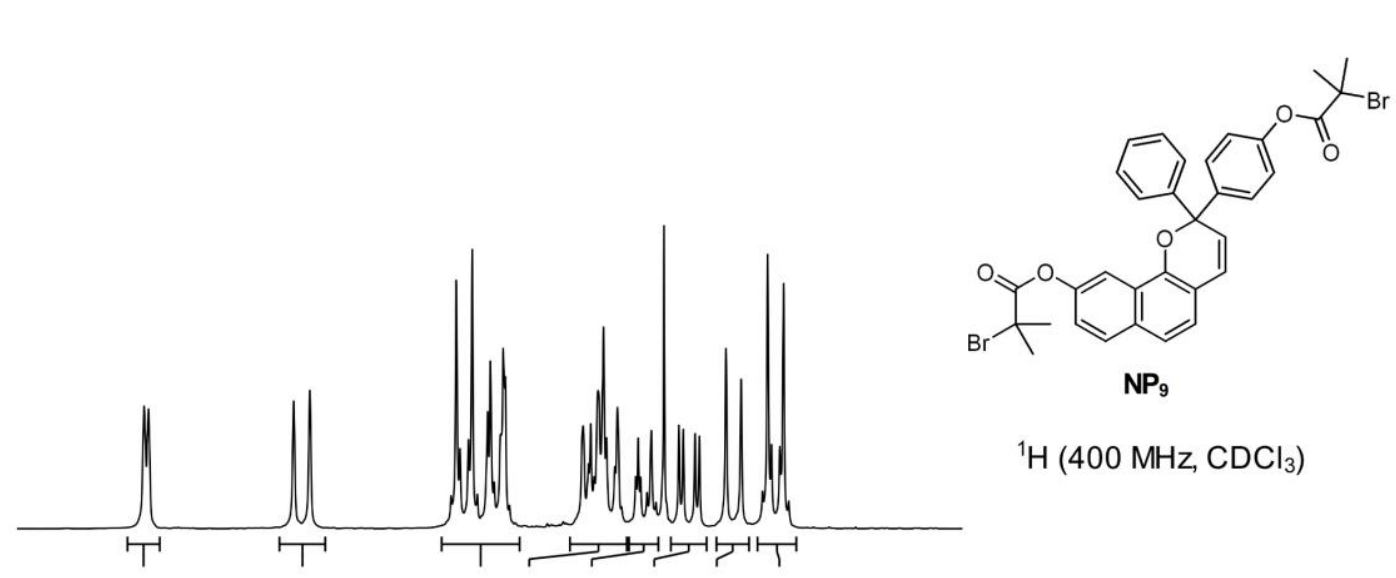

${ }^{1} \mathrm{H}\left(400 \mathrm{MHz}, \mathrm{CDCl}_{3}\right)$
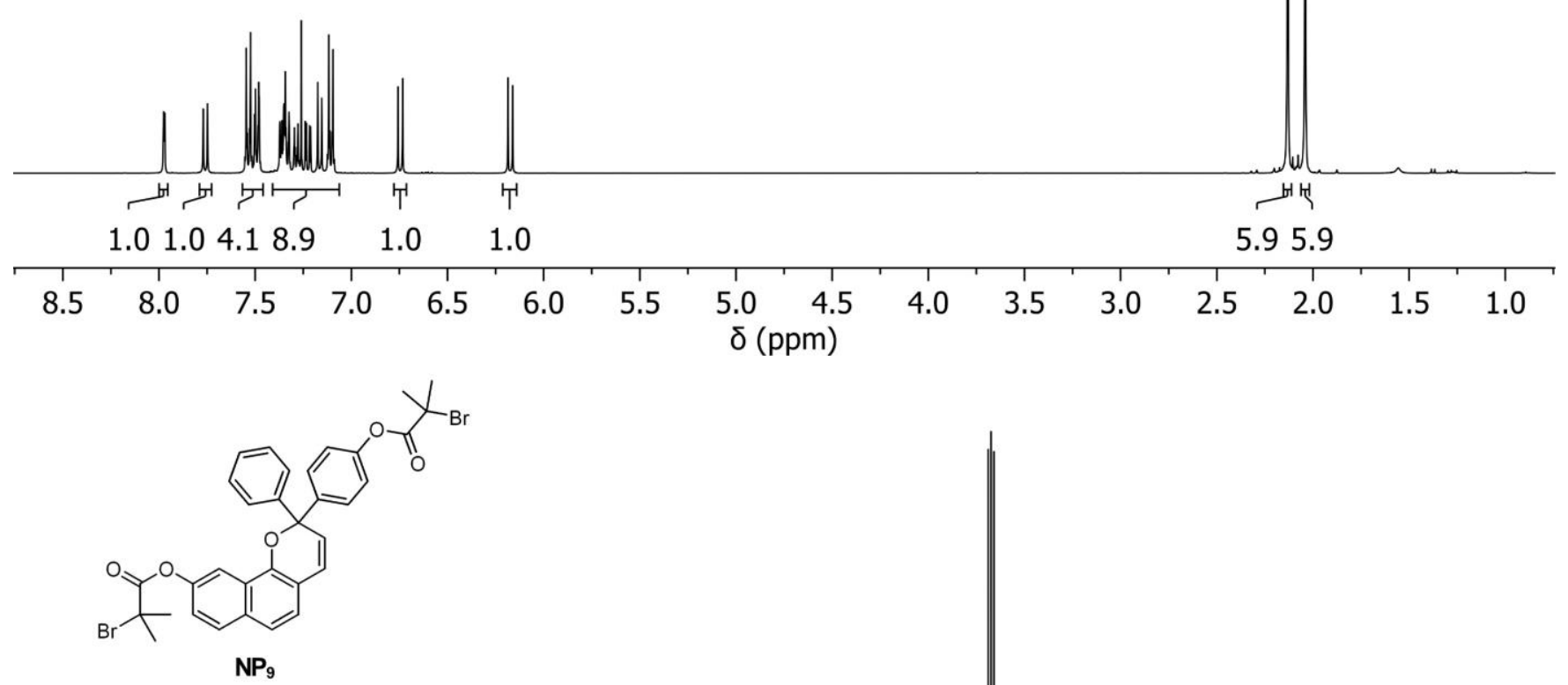

${ }^{13} \mathrm{C}\left(101 \mathrm{MHz}, \mathrm{CDCl}_{3}\right)$

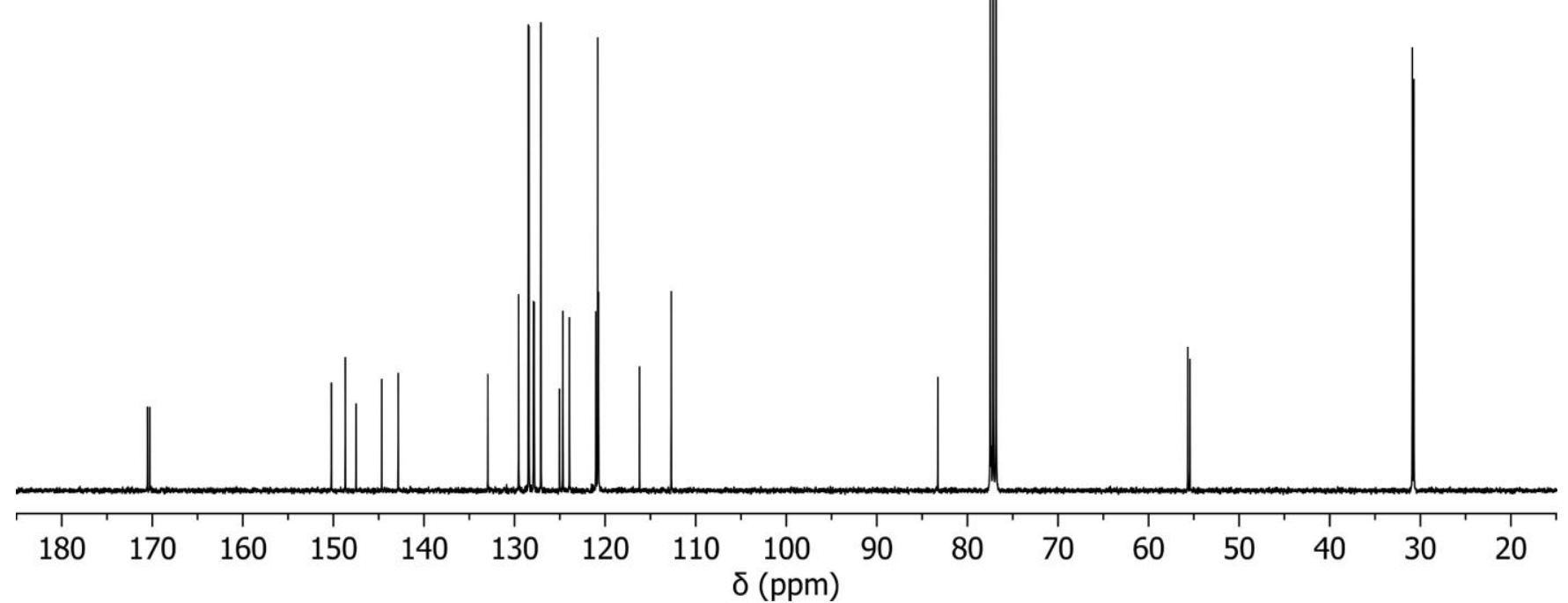




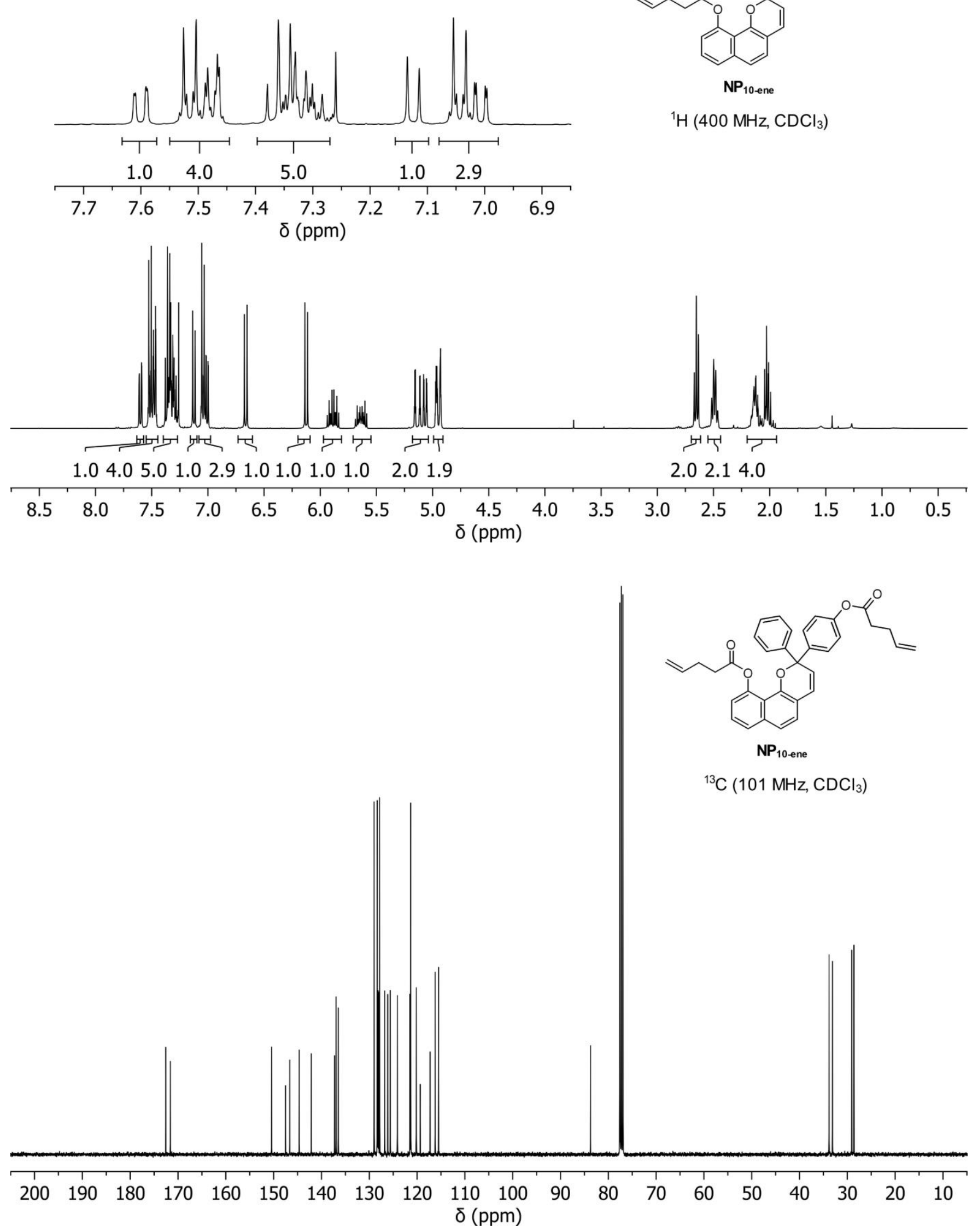




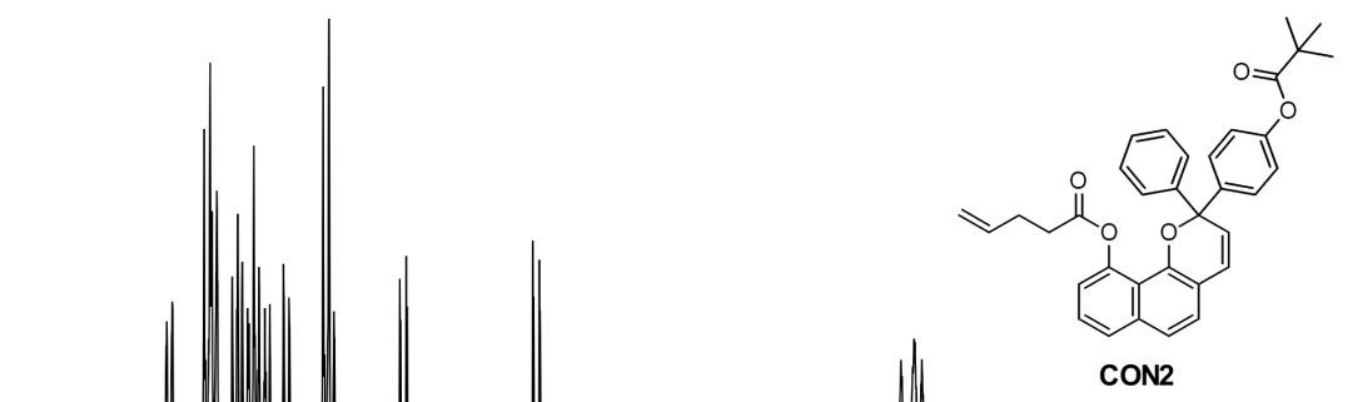

${ }^{1} \mathrm{H}\left(400 \mathrm{MHz}\right.$, acetone- $\left.d_{6}\right)$

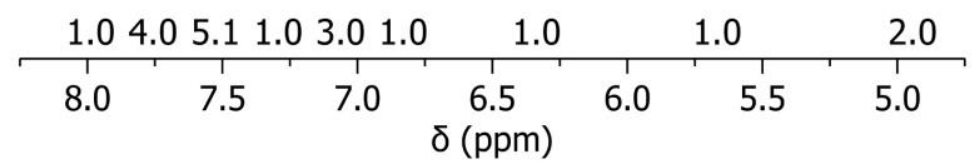
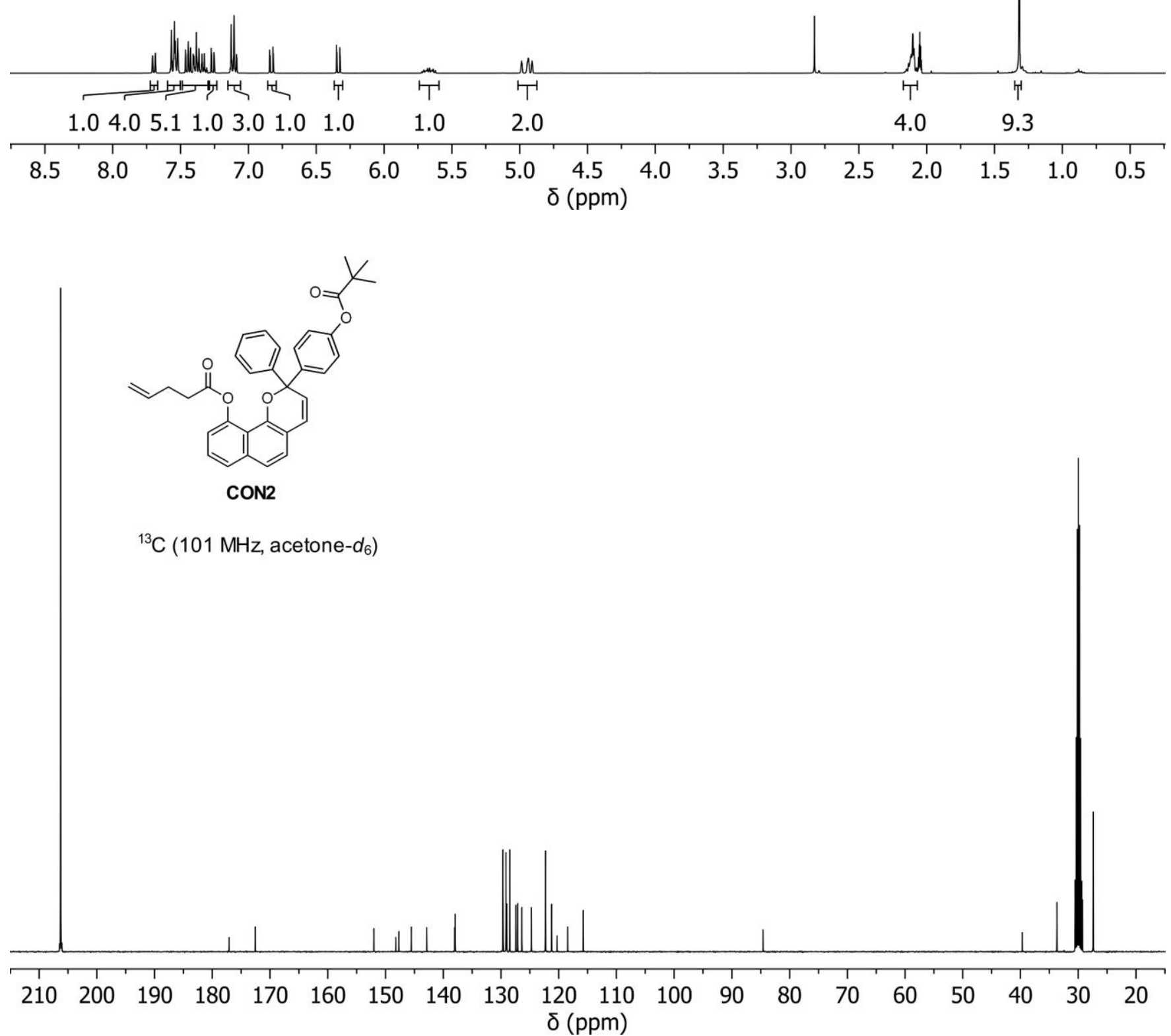

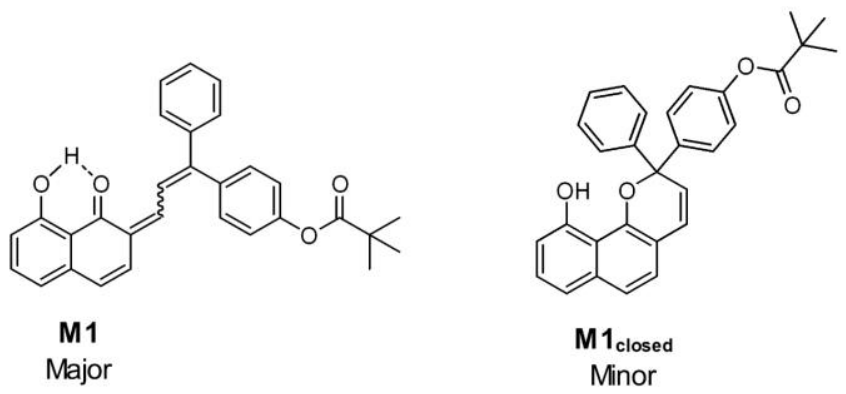

${ }^{1} \mathrm{H}\left(400 \mathrm{MHz}\right.$, acetone- $\left.d_{6}\right)$

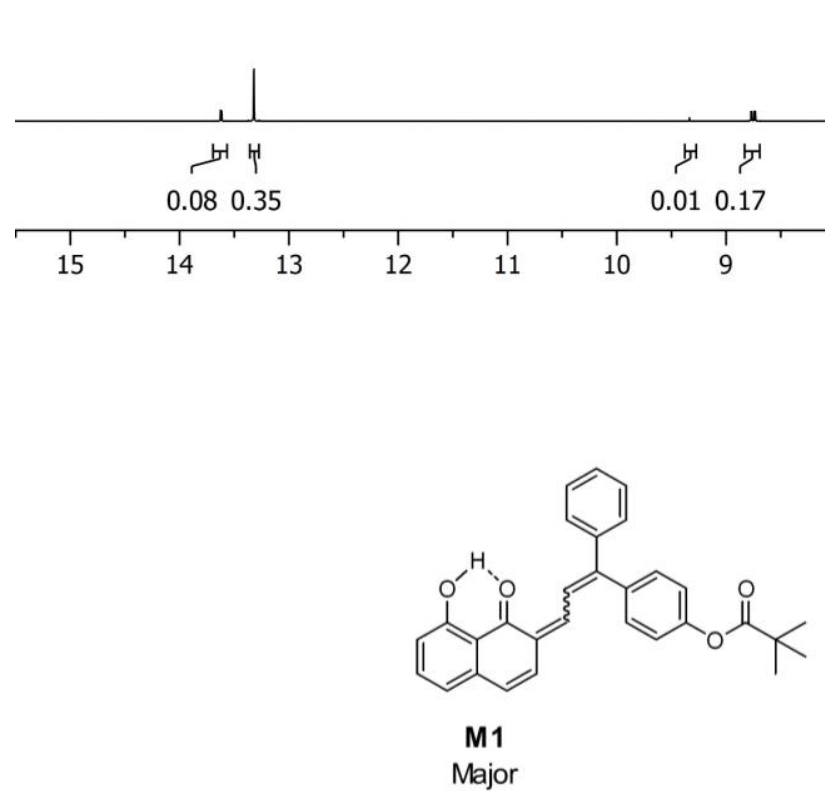

${ }^{1} \mathrm{H}\left(400 \mathrm{MHz}\right.$, acetonitrile- $\left.d_{3}\right)$

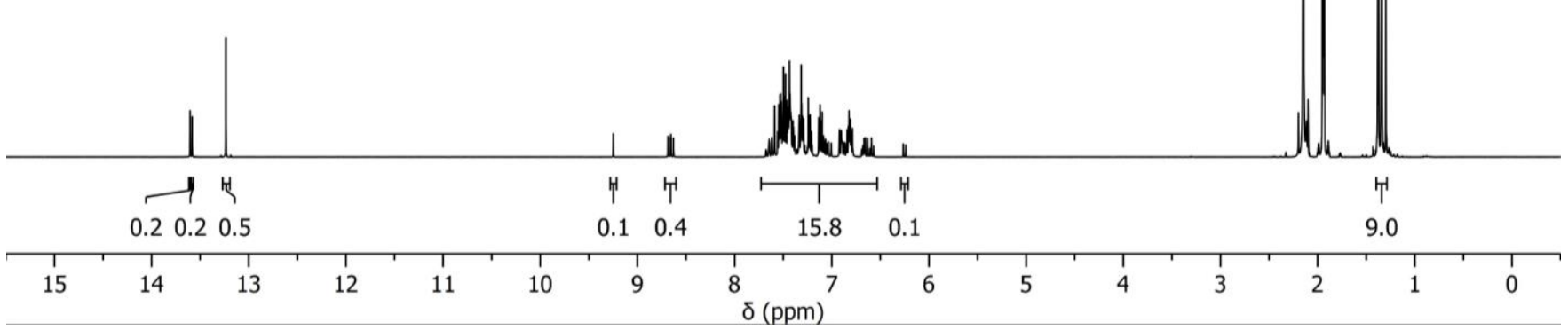

S40 


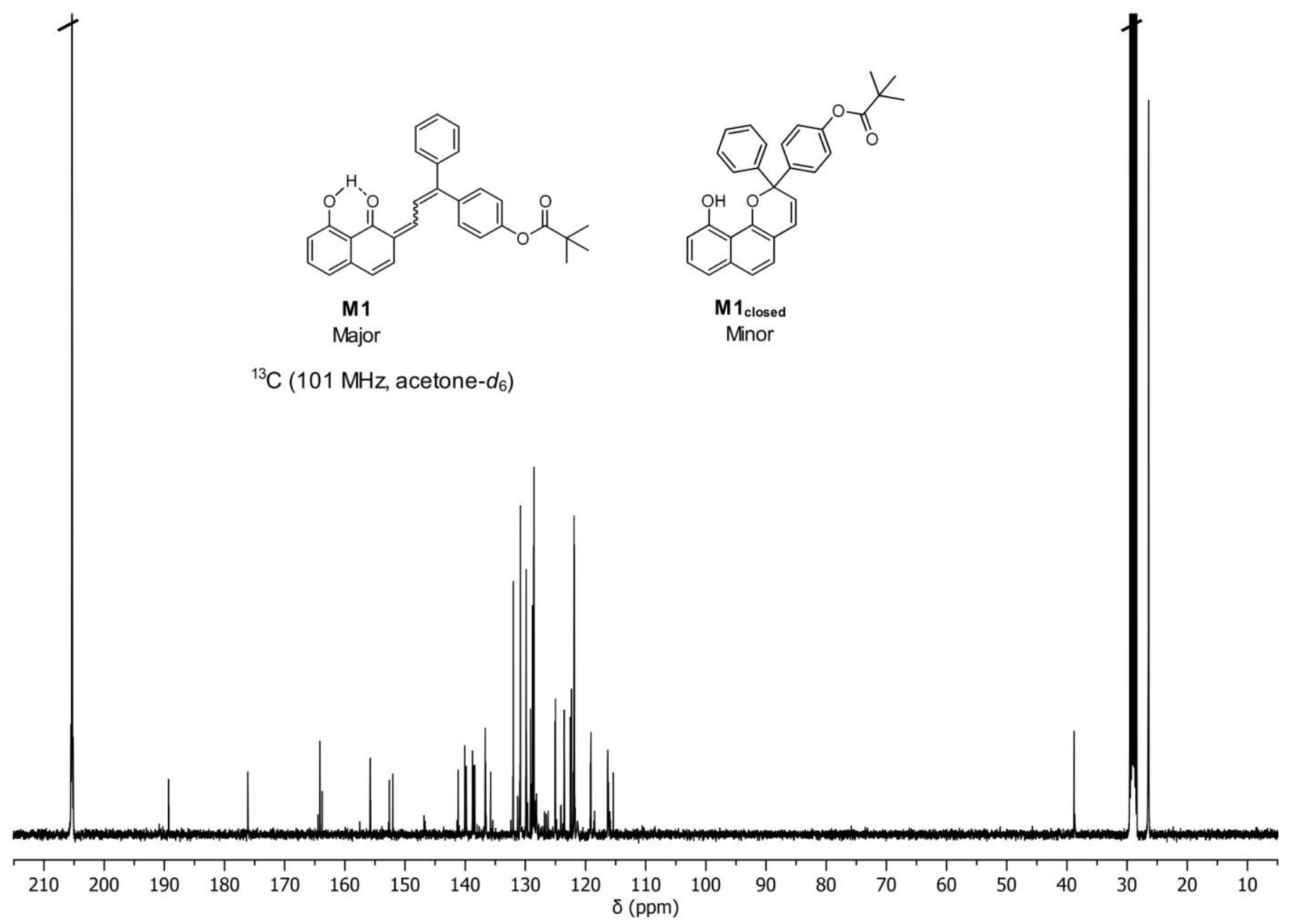

